九州大学学術情報リポジトリ

Kyushu University Institutional Repository

Computational analysis for the human eye injury using an experimentally verified anatomicalbased model

アリレザ，キャリミ

https://doi.org/10.15017/1807025

出版情報 : 九州大学，2016，博士（工学），課程博士 バージョン:

権利関係 : 全文ファイル公表済 


\title{
COMPUTATIONAL ANALYSIS FOR THE HUMAN EYE INJURY USING AN EXPERIMENTALLY VERIFIED ANATOMICAL-BASED MODEL
}

\author{
By
}

Alireza Karimi

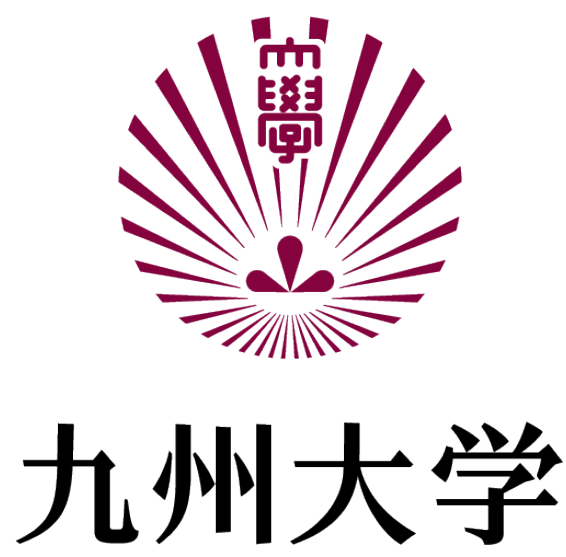

To the

DEPARTMENT OF MECHANICAL ENGINEERING

GRADUATE SCHOOL OF ENGINEERING

\section{KYUSHU UNIVERSITY}

Fukuoka, Japan

March, 2017 


\section{TABLE OF CONTENTS}

\section{TABLE OF CONTENTS \\ NOTATION}

\section{Contents}

Chapter 1: Introduction....................................................................1

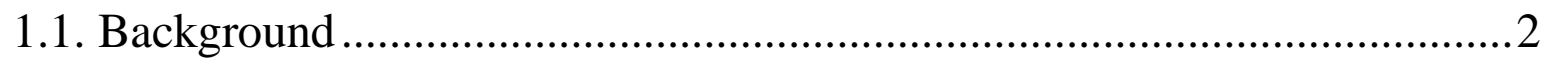

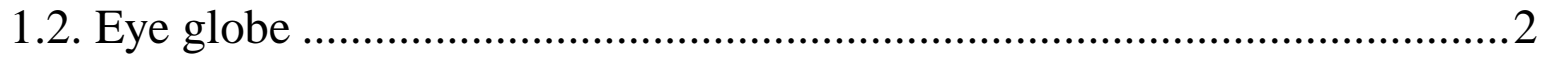

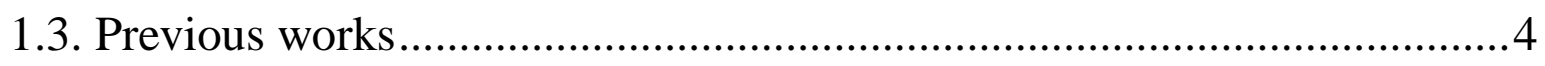

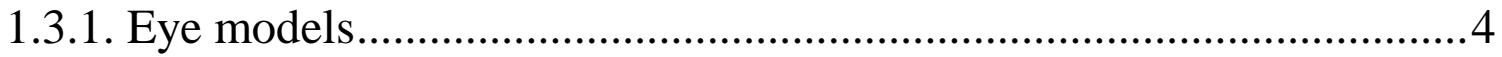

1.3.2. Sclera mechanical properties ........................................................

1.3.3. Intraocular pressure increasing in the eye ........................................ 9

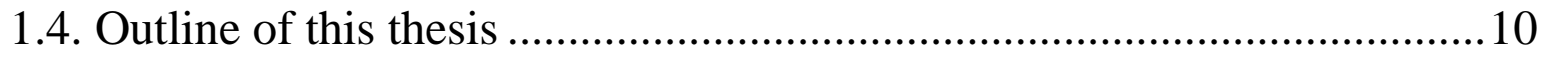

Chapter 2: Mechanical Properties of the Sclera ............................20

2.1. Sclera preparation, mechanical testing, digital image correlation technique

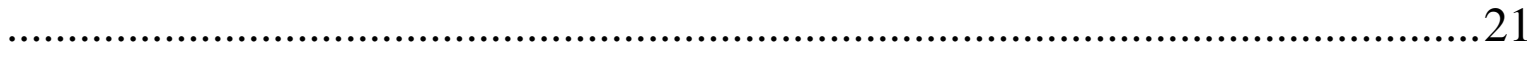

2.2. Linear elastic and nonlinear hyperelastic models ................................26

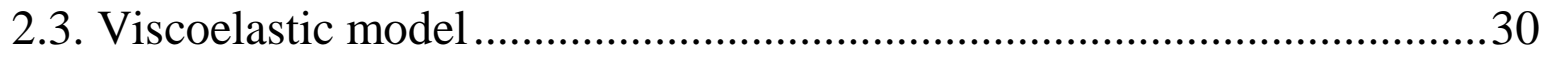

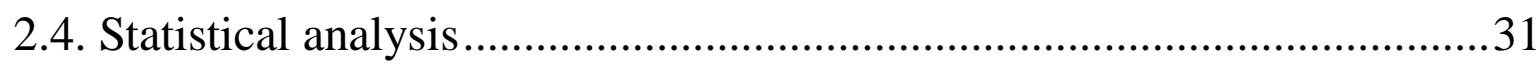

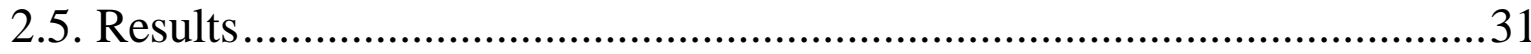

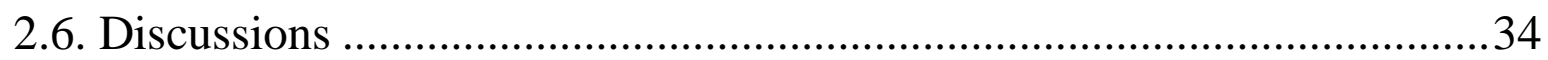

Chapter 3: Computational Model of the Eye ...............................55

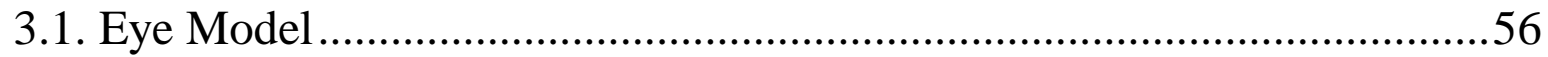

3.2. Fluid-structure interaction coupling/arbitrary lagrangian-eulerian .........60

3.3. Penetrating to the eye globe under blunt loading ....................................63

3.3.1. Penetrating test procedure ...............................................................63

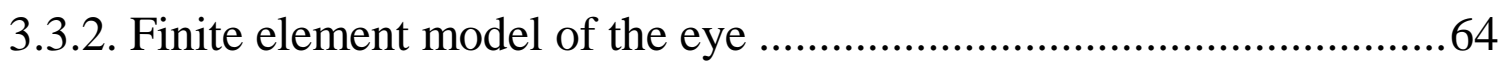

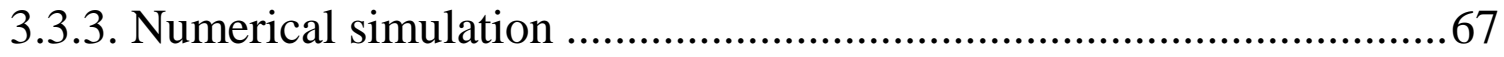




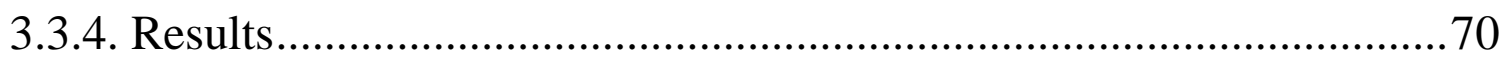

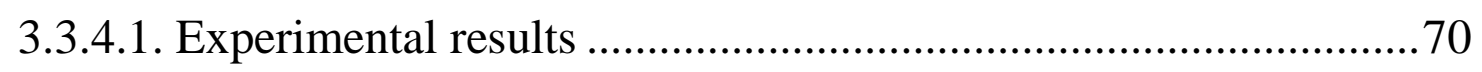

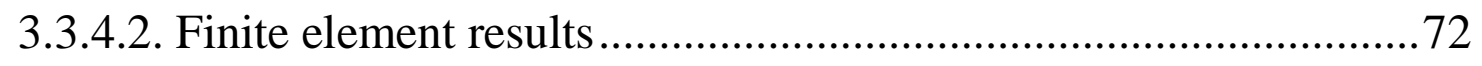

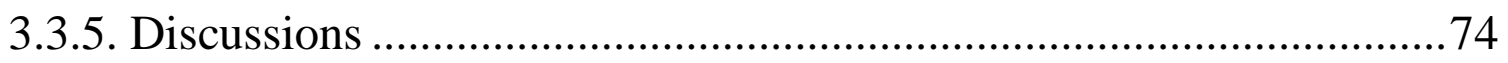

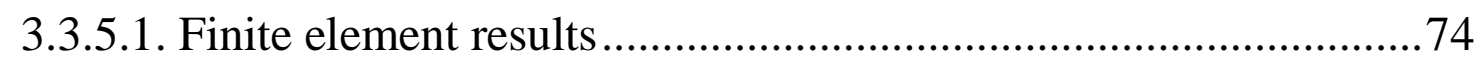

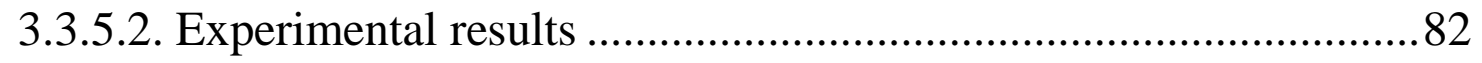

Chapter 4: Application of the Anatomical-Based Eye Model in the Anterior and Posterior Injuries ................................................ 104

4.1. Anterior injury (Tennis ball impact) ....................................................... 106

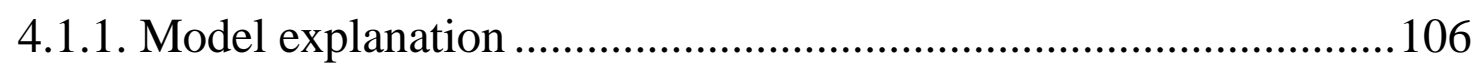

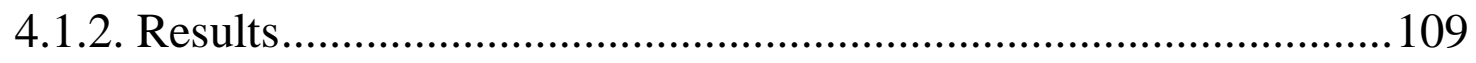

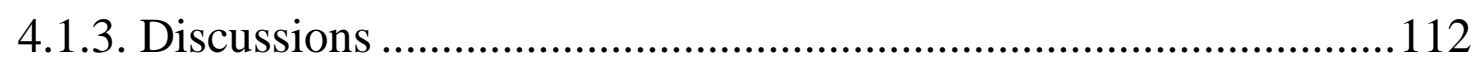

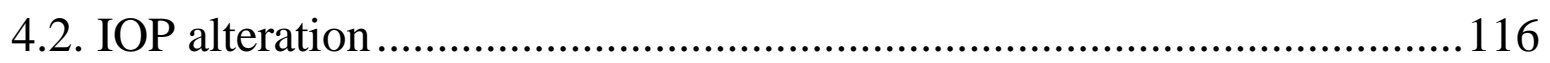

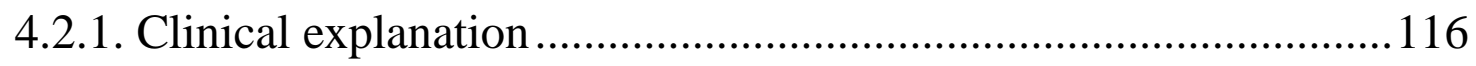

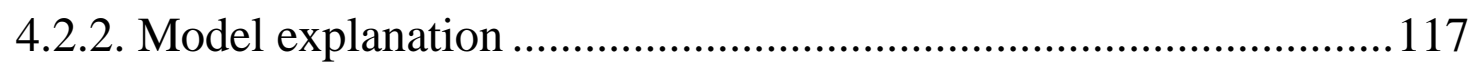

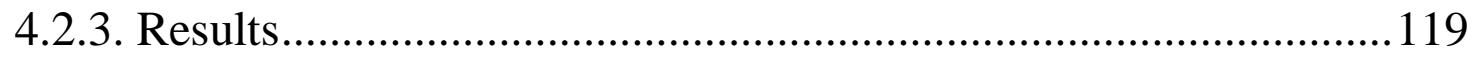

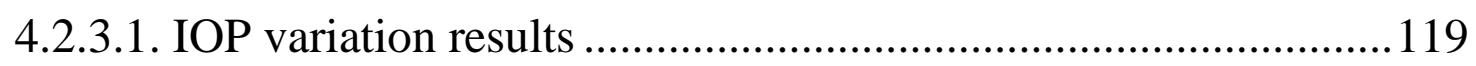

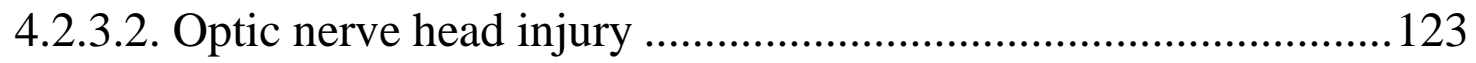

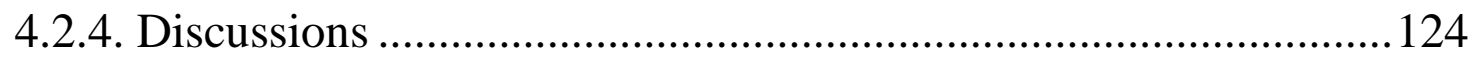

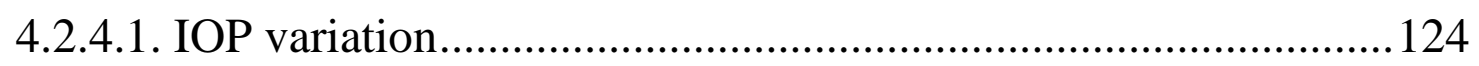

4.2.4.2. Optic nerve head biomechanics ..............................................127

Chapter 5: Conclusions........................................................... 152

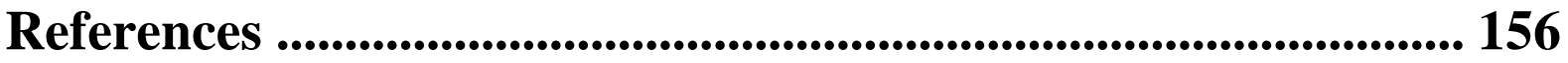

Acknowledgements ........................................................... 170 


\section{NOTATION}

FEM: Finite Element Method

FSI: Fluid-Structure Interaction

IOP: Intraocular Pressure

IVI: IntraVitreal Injection

ONH: Optic Nerve Head

SEDF: Strain Energy Density Function

MRI: Magnetic Resonance Imaging

CT: Computational Tomography 


\section{Chapter 1 \\ Introduction}




\subsection{Background}

WHO Blindness Data Bank reported annually 55 million eye injuries throughout the world which around 2.3 and 19 million of them lead to bilateral and unilateral low vision or blindness, respectively [1]. Eye injuries usually imposing a severe over-pressure to the eye globe which can be accompanied by a temporary or permanent harsh injuries to the eye components. The most common injuries in this regard have been reported to be related to the corneoscleral perforation, sphincter pupillae tear, retinal dialysis and detachment, choroidal rupture, vitreous haemorrhage, and scleral rupture [2-4]. Since in almost all the cases understanding the detail of injury to the eye is not plausible to be studied experimentally in all the components of the eye, there is a need to find a suitable approach to allow to precisely measure the intensity of injury in each of the components. Numerical methods, such as Finite Element (FE), can be useful since it allows to compute the amount of stresses and deformations in all the components under various loading conditions.

\subsection{Eye globe}

The eye globe is a structure that composes a very small area of the face while is the most vulnerable part of the face during the injury. The eye globe is consisted of many different eye components, such as the iris, cornea, sclera, iris, lens, retina, optic 
nerve, etc., as displayed in Fig. 1. Each component of the eye has its own performance accompanied with its own mechanical properties. In this section, it is aimed at understanding the anatomical location of each component of the eye to help to shed light on the interaction and load bearing of each component of the eye. However, before that it was needed to know the location of each component of the eye in the globe to be able to not only propose a better eye model but also figure out the load bearing of each of components of the eye.

The sclera is a collagenous tissue which is the holder of the intra components of the eye and covers most part of the eye components up to the end of the Optic Nerve Head $(\mathrm{ONH})$ [5]. However, any types of the loading whether from the inside or outside of the eye due to its over-pressure can severely deform the anterior or posterior components of the eye. The role of the sclera in this regard is deemed important since it nourishes the optic nerve from its posterior side thru the ciliary arteries [6]. In addition, it has been reported that the sclera has a crucial role in the biomechanics of the $\mathrm{ONH}$ during the load applying to the eye globe [7]. On the other hand, any deformation or dislocation in the attachment site of the optic nerve and the sclera, namely ONH, can significantly affect the vision acuity [8]. Therefore, it is of great importance to consider the role of sclera and its mechanical properties directly into the eye to be able to investigate the injury to the $\mathrm{ONH}$. The ciliary body in here controls the shape and deformation of the lens as they are in direct contact 
with each other. The complexity of the anterior components of the eye is presented in Fig. 2. The optic nerve transmits the signal to the brain for vision making thru its attachment site to the sclera, namely $\mathrm{ONH}$. The aqueous body is a transparent fluid like material in the anterior side of the eye which maintains the intraocular pressure. The lens is a biconvex transparent structure in the anterior side of the eye which is in charge of accommodating the curvature to provide a focusing in the macula in the end of the eye. The cornea also is a transparent structure of the eye which is a light focuser in the most anterior side of the eye. The iris is a thin structure in the anterior side of the eye which is in charge of proving a diaphragm for light absorption according to the level of light in the room. The vitreous body is a gel structure which fills the space between the lens and retina in the eye ball. Choroid is a thin vascular layer of the eye which is in charge of nourishing the circumference components of the eye.

\subsection{Previous works}

\subsubsection{Eye models}

Various eye models so far on a basis of Computed Tomography (CT)/Magnetic Resonance Imaging (MRI) data as well as the anatomical features of the eye have been proposed. Each of them has its own pros and cons. Since the dimensions of the eye may vary according to the ethnicity of the people [9], using the CT/MRI data is 
preferable to measure the anatomical characteristics of the eye according to the ethnicity or demographic condition of the local people. Hence, having CT/MRI data in this regard would help to establish a more suitable eye model for simulation purposes. On the other hand, the CT/MRI data may not be able to provide the detail anatomical and morphological characteristics of all the components of the eye and this may impose a difficulty into the eye model. There are four common well-known models of the human eye, including Uchio et al. [10, 11], Stitzel et al. [12], Rossi et al. [13], and Lovald et al. [14]. In the model proposed by Uchio, the shape of the sclera which is the most outer component of the eye was extracted from the CT/MRI data whereas the characteristics of the rest of the components, including scleral thinning and the nonlinear cornea, were obtained from the literature [15]. The lens was modeled as a rigid body with its mass accounted for. The presence of retina was ignored and the vitreous bodies were considered to be simple elastic. In addition, in their model some of the components of the eye were supposed to be made of a unique solid element with only a mass, such as the iris, ciliary body, and aqueous body. Another issue is the lack of thorough validation for their proposed model. This was why the applications of their model to use for injury prediction in traumatic-impact scenarios were almost limited. The Uchio's eye model is presented in Fig. 3.

Another model almost at the same time proposed by Stitzel et al. Their model (Virginia Tech Eye Model (VTEM)) although did not consider some of the 
components of the eye, such as the retina and iris, it was experimentally verified via a baseball impact to the eye. However, it should be noted that in their experimental verification, only the amount of stress as a result of injury in the anterior/exterior wall of the eye globe was computed. Therefore, the amount of stresses and deformations in the rest of the components have not taken into account and have not been compared which may affect the validity of the model. Since there was a fluid component in their eye model, their simulations were carried out on a basis of FluidStructure Interaction (FSI) approach. That is, the deformation of the aqueous body and vitreous body as the fluid components transferred to the other components of the eye as the solid components. The Stitzel's eye model is displayed in Fig. 4.

Thereafter, Rossi et al. in 2011 proposed an eye model. Basically, this model was established on a basis of $3^{\text {rd }}$ model. That is, in this model exactly the same components that have been ignored in the $3^{\text {rd }}$ model were ignored. In addition, the mechanical properties of the lens and ciliary body were considered the same. The $3^{\text {rd }}$ model considered the role of zonule and ciliary body separately, however, in the Rossi's model the role of zonule was ignored. In addition, no experimental analyses have been carried out to validate their proposed model. The Rossi's FSI eye model is presented in Fig. 5 .

Finally, in 2017 Lovald et al. performed a combination of FE analysis and experimental estimation to shed light on the injury of the eye globe thru their 
proposed eye model. The iris and retina were ignored in their model; and the vitreous body as well as aqueous body were considered to be a fluid cavity with a hydrostatic pressure. The model proposed by Lovald et al is presented in Fig. 6. The mechanical properties of the eye components in the eye models in addition to the provided information are listed in Table 1.

To sum up, it is understood that each eye model despite its ability to simulate the injury to the human eye, has its own lacking by not considering some of the components of the eye.

\subsubsection{Sclera mechanical properties}

To have suitable geometry or anatomical dimensions for an eye model, an eye model also needs to be enriched with a set of appropriate material models to be able to produce accurate reliable results. Sclera is a collagenous eye components which has a relatively high elastic modulus $(1.66 \pm 0.35 \mathrm{MPa}(\mathrm{Mean} \pm \mathrm{SD}))$ among the other components of the eye. It also acts as a holder/container of the intra components of the eye when it subjects to a mechanical deformation during any types of injuries. So far, there are substantial amounts of efforts to characterize the mechanical properties of the scleral tissue to be incorporated into FE models of the eye [16-20]. However, these studies whether mostly used animal samples or performed the test under compressive loadings to have the quantities of the elastic or hyperelastic 
properties of the sclera tissue. It was reported that the average porcine scleral modulus is $75 \%$ less than that of the measured one for the human tissue $(0.65 \pm 0.53$ $\mathrm{MPa}$ versus $2.60 \pm 2.13 \mathrm{MPa}(\mathrm{Mean} \pm \mathrm{SD})$, respectively) [16]. Therefore, using animal models instead of the human ones can affect the numerical outcomes of an eye model. Moreover, it is known that the sclera almost always subjects to a tensile loading during load bearing. Furthermore, it should be noted that the reported elastic modulus for the human sclera tissue varied considerably among the studies as 0.15 0.83 [16], 0.2-0.5 [21], 2.6 [22], 2.9 [23], $358 \mathrm{MPa}$ [24]. This variation among the elastic modulus can affect the numerical results of an eye model. For example, it has been reported that the stiffening of the sclera largely influences the IntraOcular Pressure (IOP) and, as a result, largely intensify the injury to the $\mathrm{ONH}$ [7]. Meanwhile, since the sclera is the only component of the eye which transfers the load into the ONH [7], its mechanical properties substantially affect the vision acuity [25]. In addition, all the results about the mechanical properties of the sclera tissue only reported for single loading rate whereas it is known that the sclera subjects to various loading at different rates according to the impacted object. Hence, it was needed to distinguish the tensile mechanical properties of the human sclera tissue under various loading rates to be able to propose a suitable eye model on a basis of injuries' loading rates. On the other hand, numerical modeling results so far revealed 
that understanding the mechanical properties of the sclera would improve our knowledge about the ONH [26] and its effects on the surrounding tissues [27].

\subsubsection{IOP increasing in the eye}

It is obvious that all components of the eye involve to provide vision, but the cornea in the anterior and optic nerve in the posterior sides of the eye globe provide the most remarkable roles by power focusing and transferring electrical signals to the brain, respectively [28]. The cornea is also in charge of protecting the eye from outer matter and tolerates IOP from the inside. Consequently, it is anticipated that even a very trivial variation in the morphology of the cornea would considerably affect the performance and superiority of the vision [29].

The IOP in the aqueous body can change and induce substantial deformation in other eye components, especially the cornea, lens, and the optic nerve. Regarding the cornea, in a keratoconus cornea the localized loss of corneal thickness invoke the weakened cornea bulges outward because of not being able to bear IOP in its back side [30] which invokes gradually conical shape of the cornea and, as a result of that, a blurred vision [31]. However, there is a lack of knowledge on the role of IOP alterations on the structure and shape of the cornea and also other components of the human eye. 


\subsection{Outline of this thesis}

In this thesis, it was objective to propose a suitable computational anatomicalbased model of the human eye to be used in eye injury simulation of both the anterior and posterior components of the eye. The anterior injury can be named as blunt trauma impact while the posterior injury can be called as increasing the IOP. Each of these injuries can subject the components of the eye to a severe deformation which are difficult to be diagnosed by ophthalmologists. Therefore, using another approach which can broaden the vision of doctors in this regard is of crucial important. Numerical Finite Element (FE) method is this regard can be applicable since it allows to compute the amount of stresses and deformations in all the components of the eye in detail under various loading conditions. A suitable computational eye model not only should have benefitted from a set of proper mechanical properties and anatomical geometry, but also it should be experimentally and numerically verified. To strengthen the material parts of the model, the mechanical properties of the sclera were experimentally measured and incorporated into the model.

In chapter 2, it was pointed out that the sclera has a pivotal mechanical role in the eye globe in terms of the load bearing as well as ONH biomechanics. The linear elastic, nonlinear hyperelastic, and linear viscoelastic mechanical properties of the sclera tissue were determined. In addition, since the cornea during the injury subjects to various loading conditions, having the mechanical properties of the sclera at 
various rates pave the way for us to propose a more accurate model accompanied with further accurate numerical outcomes.

In chapter 3, using the CT/MRI/literature data, an anatomical-based eye model was established which was consisted of the cornea, aqueous body, iris, lens, vitreous body, ciliary body, sclera, retina, optic nerve, intra and extraconal fats, and muscle. Especially, the morphology of the cornea, iris/aqueous body, lens, vitreous body/retina/sclera, muscles' head, and optic nerve were determined thru the CT/MRI data. In order to validate the accuracy of the model, the cornea subjected to penetrating test at various loading rates both experimentally and numerically. Furthermore, to examine the advantage of our model, four different eye models according to the literature were also simulated and compared.

In chapter 4 , the proposed anatomical-based eye model was used to investigate the injury as a result of tennis ball impact as well as the IOP alteration. Regarding the blunt trauma impact, a tennis ball at the speed of $69.29 \mathrm{~m} / \mathrm{s}$ was shoot to the eye and the amount of stresses and deformations in all the components of the eye as a result of $1 \mathrm{~mm}$ deformation in the apex of the cornea were computed. In addition, in order to consider our own measured mechanical properties of the sclera/optic nerve, the impact was simulated at two different elastic moduli of the sclera/optic nerve, including our data and reference data. Regarding the IOP alteration, three different 
IOPs, including 10,20,30 mmHg, were applied in the aqueous body and the resulted stresses and deformations in the rest of the components were all computed.

In chapter 5, the conclusion of this thesis was provided. Our anatomical-based eye model in the concurrent study has implications not only for computing the stresses and deformations in the anterior and posterior eye components as a result of mechanical deformation, but also for providing a medical insight for the ophthalmologists to figure out the detail of injury to each components when experimental diagnosis is not available. 
Table 1. A comparison between the mechanical properties of the eye components among the eye models.

\begin{tabular}{|c|c|c|c|c|}
\hline $\begin{array}{l}\text { Eye } \\
\text { components }\end{array}$ & Uchio & Stitzel & Rossi & Lovald \\
\hline Cornea & Nonlinear $^{1}$ & $\mathrm{E}=1.5 \mathrm{MPa}$ & $\mathrm{E}=1.5 \mathrm{MPa}$ & Nonlinear $^{1}$ \\
\hline $\begin{array}{l}\text { Aqueous } \\
\text { body }\end{array}$ & Solid mass & $\mathrm{EOS}^{3}$ & $\mathrm{EOS}^{3}$ & $\begin{array}{l}\text { Fluid } \\
\text { cavity }\end{array}$ \\
\hline Iris & Solid mass & - & - & - \\
\hline Ciliary body & Solid mass & $\mathrm{E}=11 \mathrm{MPa}$ & $\begin{array}{c}\text { Bulk modulus }=1000 \\
\mathrm{MPa}\end{array}$ & $\begin{array}{c}\mathrm{E}=11.2 \\
\mathrm{MPa}\end{array}$ \\
\hline Lens & Rigid body & $\mathrm{E}=6.88 \mathrm{MPa}$ & $\begin{array}{c}\text { Bulk modulus }=1000 \\
\mathrm{MPa}\end{array}$ & $\begin{array}{c}\mathrm{E}=6.92 \\
\mathrm{MPa}\end{array}$ \\
\hline Zonules & - & $\begin{array}{c}\mathrm{E}=357.78 \\
\mathrm{MPa}\end{array}$ & 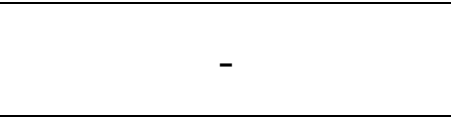 & $\begin{array}{c}E=3582 \\
M P a\end{array}$ \\
\hline $\begin{array}{l}\text { Vitreous } \\
\text { body }\end{array}$ & $\begin{array}{c}\text { Solid mass with } \\
\text { a hydrostatic } \\
\text { pressure of } \\
20 \mathrm{~mm} \mathrm{Hg}(2.7 \\
\mathrm{kPa})\end{array}$ & $\mathrm{EOS}^{3}$ & $\mathrm{EOS}^{3}$ & $\begin{array}{l}\text { Fluid } \\
\text { cavity }\end{array}$ \\
\hline Retina & 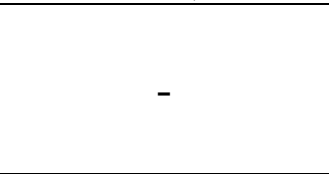 & - & $\begin{array}{c}\text { Shear modulus }=0.035 \\
\text { MPa and Bulk } \\
\text { modulus }=1000 \mathrm{MPa}\end{array}$ & - \\
\hline Sclera & Nonlinear $^{2}$ & $\mathrm{E}=5.5 \mathrm{MPa}$ & $\mathrm{E}=28 \mathrm{MPa}$ & Nonlinear $^{2}$ \\
\hline
\end{tabular}

Nonlinear in here stands for the data point obtained from the stress-strain diagram.

EOS stands for equation of state.

${ }^{1}$ Components with the same mechanical properties.

${ }^{2}$ Components with the same mechanical properties.

${ }^{3}$ Components with the same mechanical properties.

- Means this component was not considered in this eye model. 


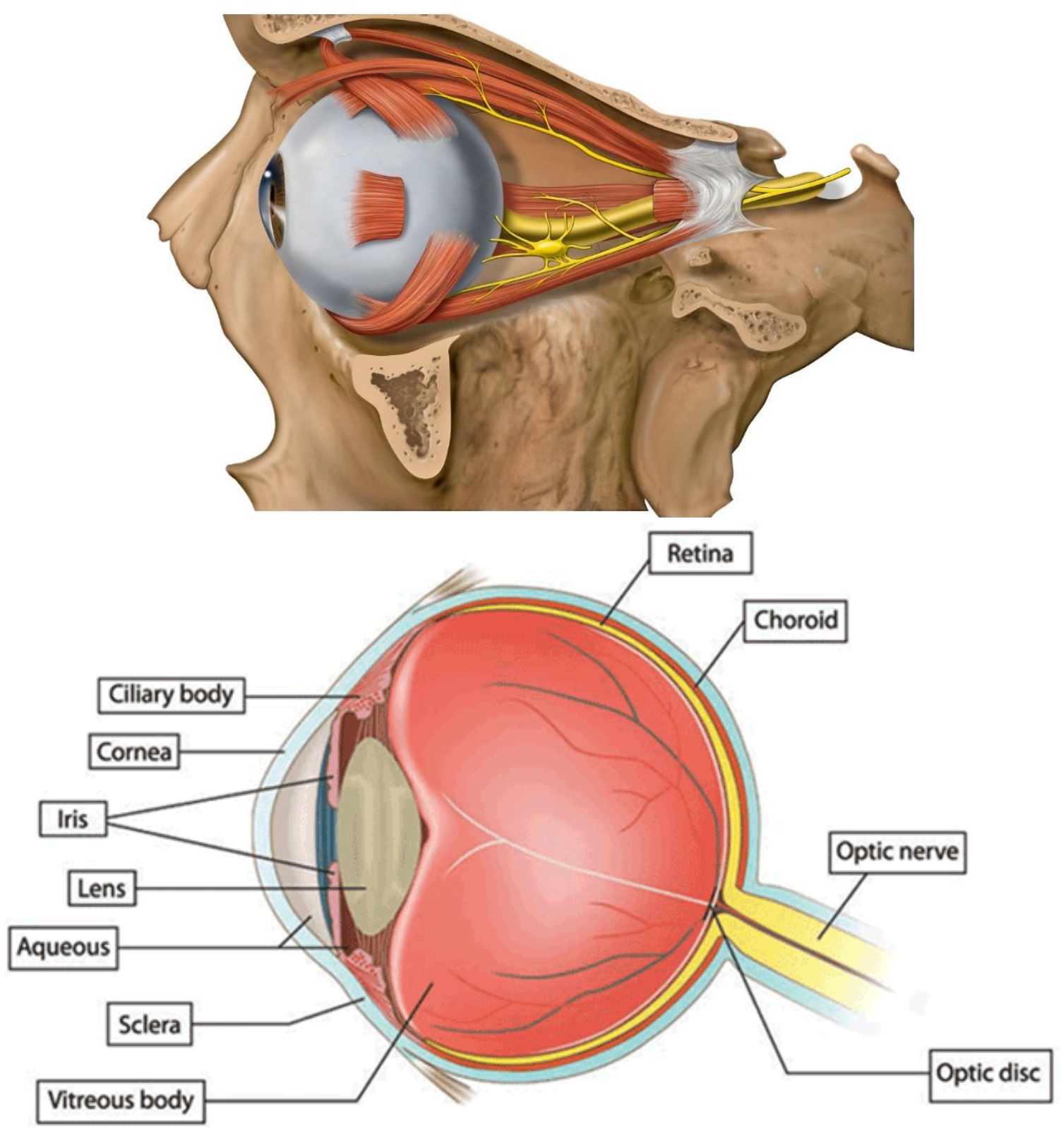

Fig. 1. The anatomical location of the eye from the side view [32]. Schematic diagram of the human eye [33]. License Number: 4034620747868 


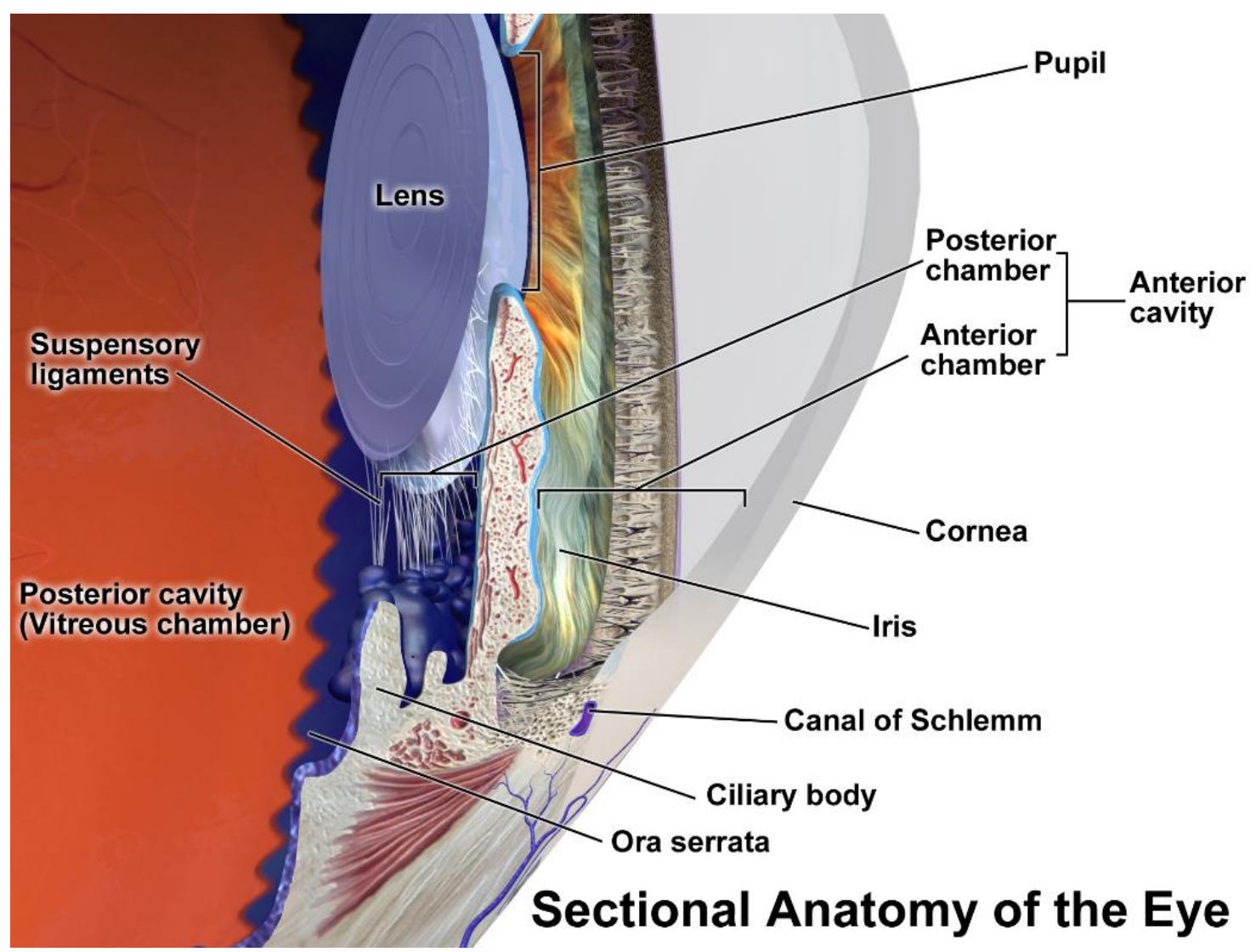

Fig. 2. Anterior part of the human eye [34]. License number: 1990-2007. 

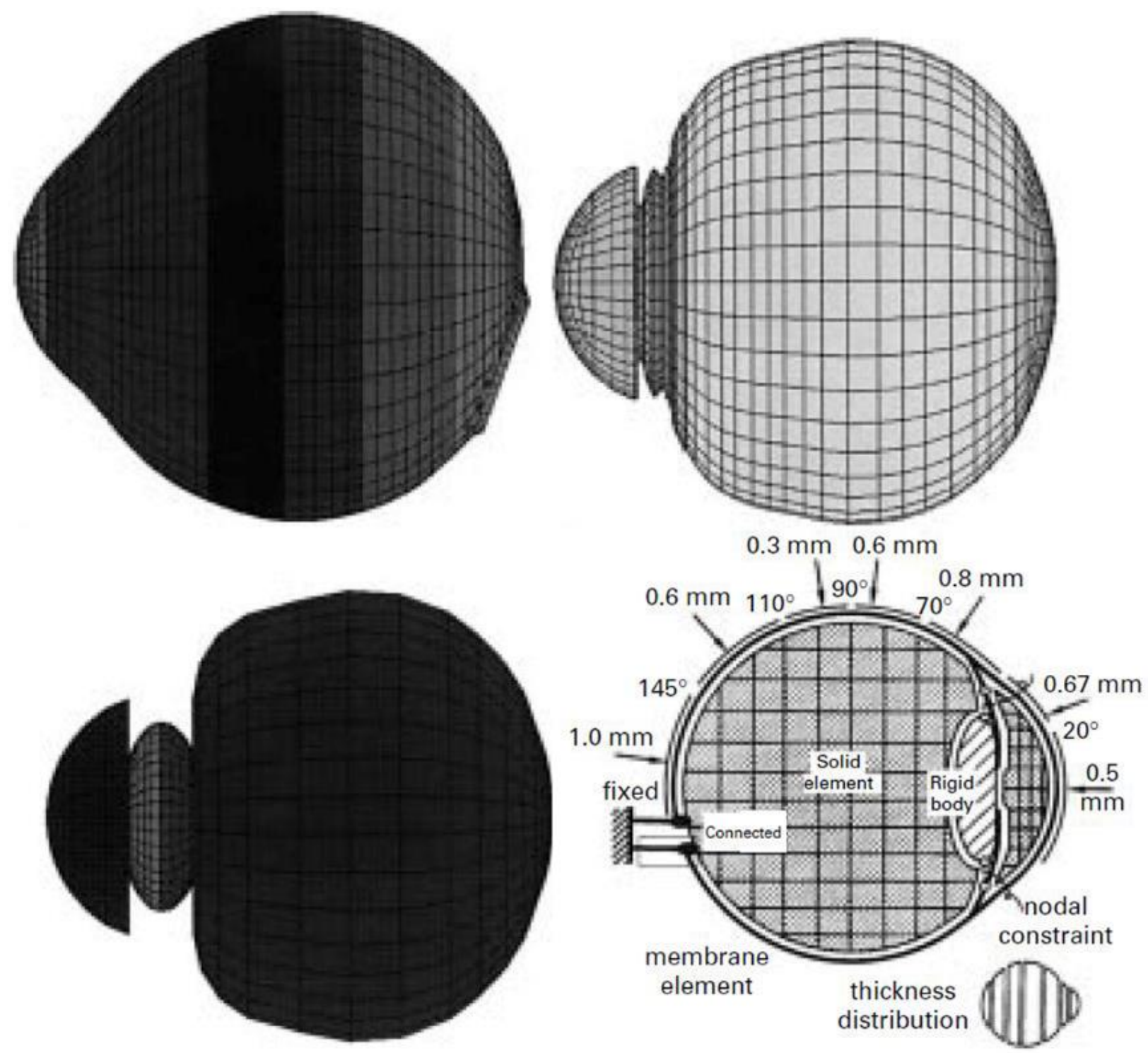

Fig. 3. The eye model of Uchio et al. [10, 11]. License Number: 4034610711007 

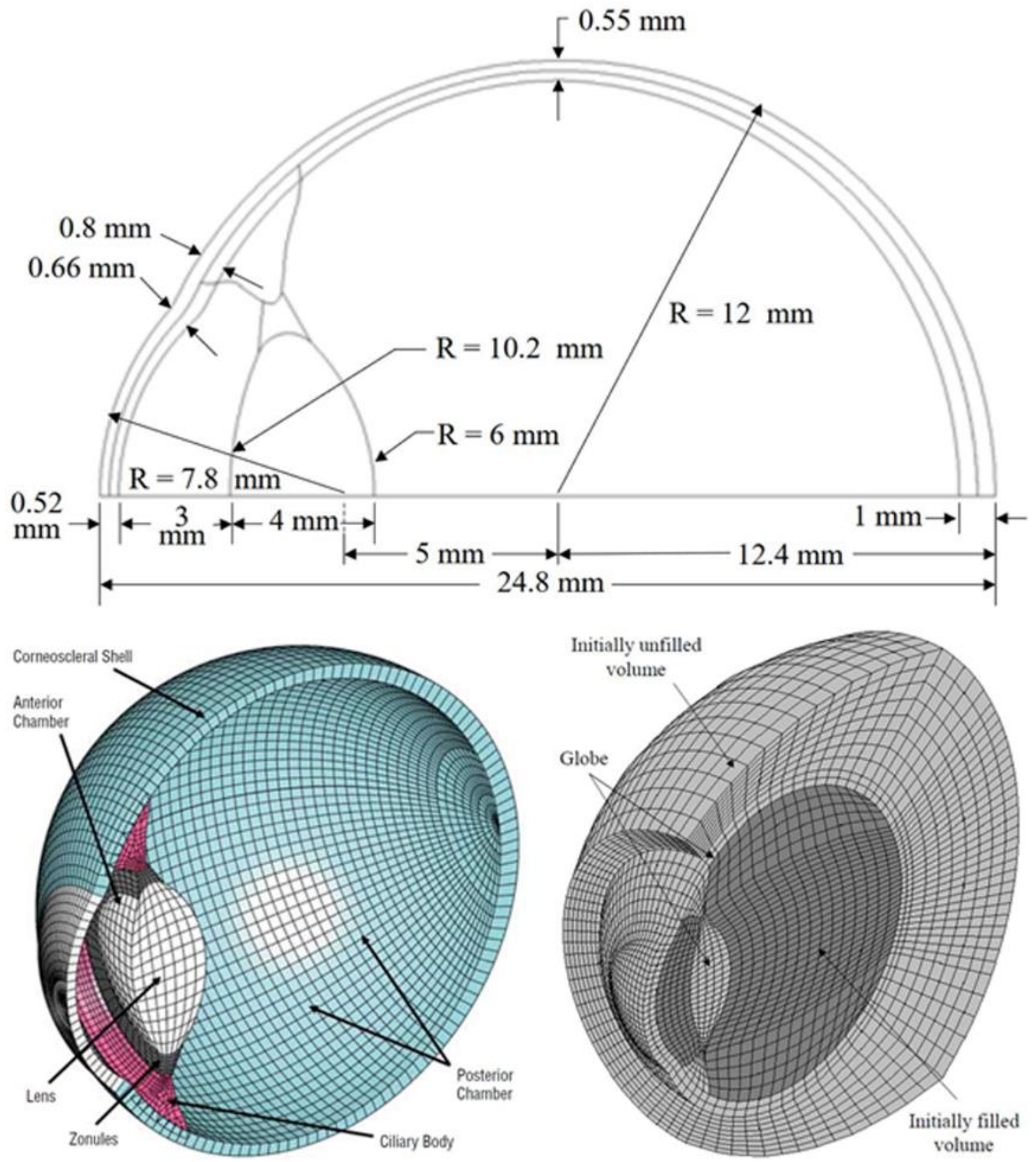

Fig. 4. The eye model of Stitzel et al. [12]. License Number: 4034620402562 


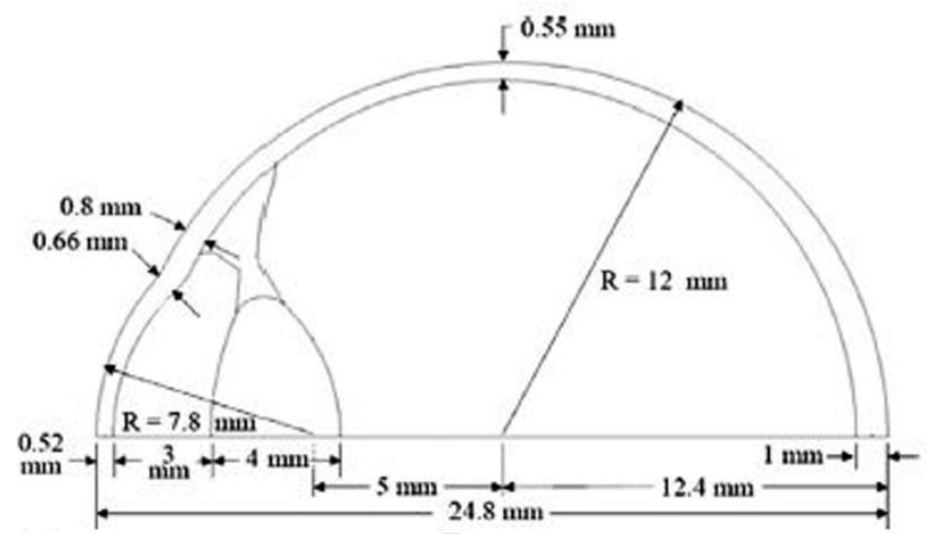

$\mathrm{E}$

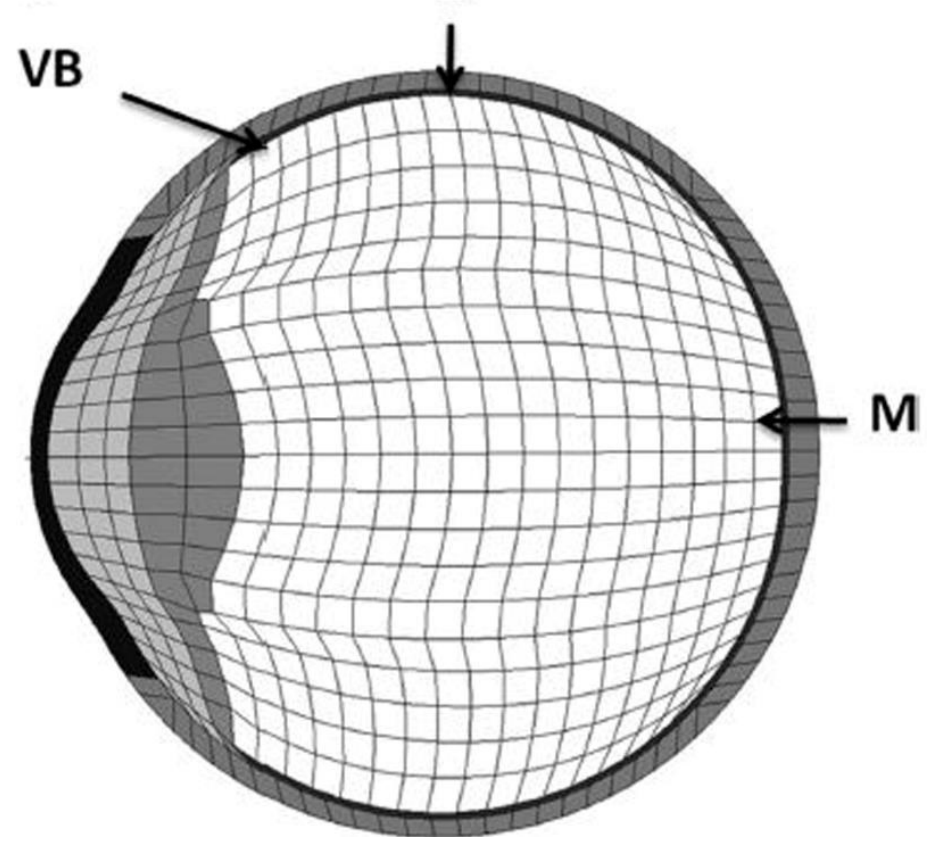

Fig. 5. The eye model of Rossi et al. [13]. License Number: 4034620402562 


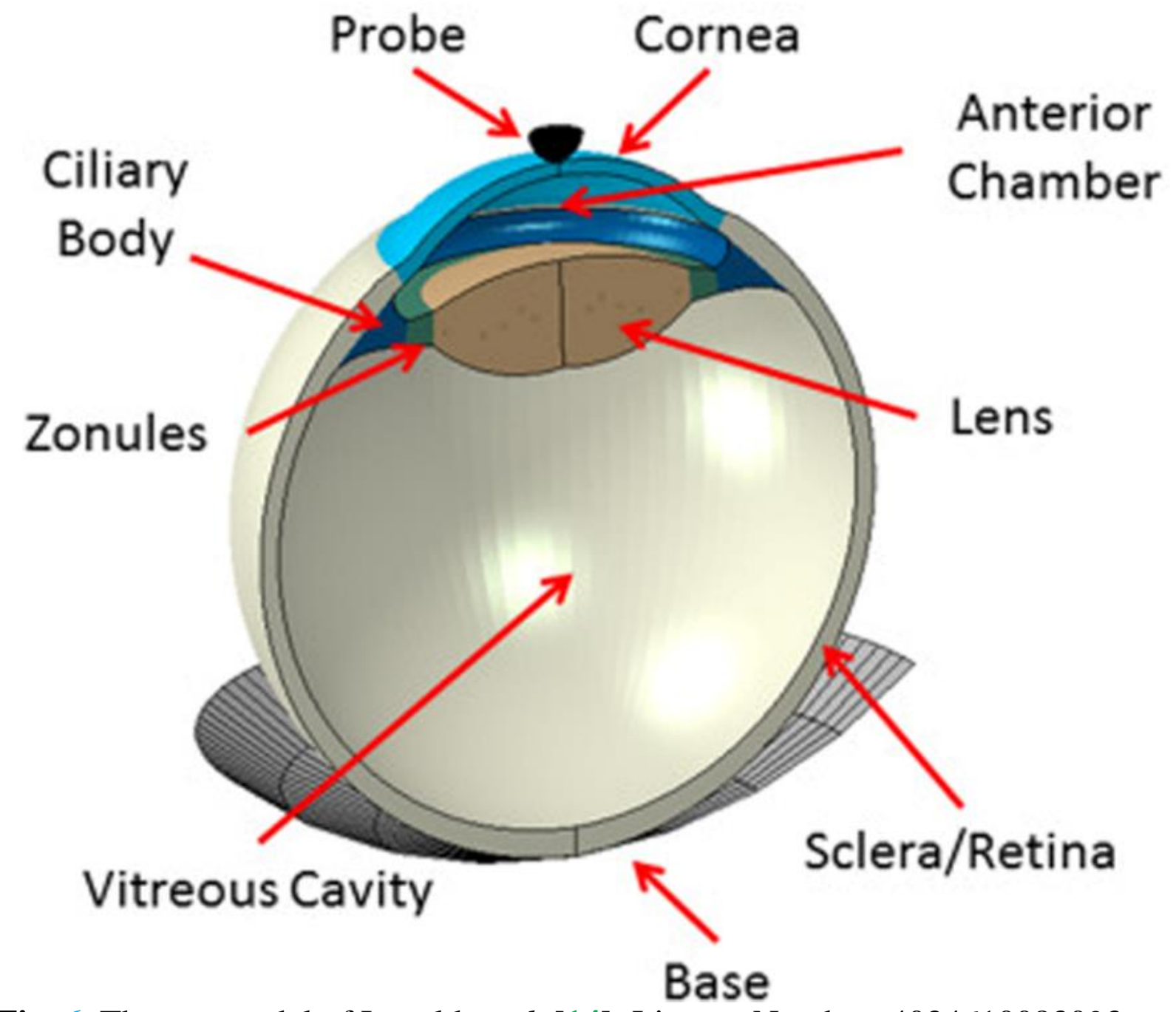

Fig. 6. The eye model of Lovald et al. [14] . License Number: 4034610083093 


\section{Chapter 2 \\ Mechanical Properties of the Sclera}


In this chapter, it is aimed at experimentally measuring the mechanical properties of the human sclera tissue to be used for incorporating into our proposed human eye. As it was pointed out, the mechanical properties of the sclera due to its crucial role in holding the intra components of the eye are of vital important as well as its pivotal role in the $\mathrm{ONH}$ biomechanics. Once a foreign body severely hits the eye or any types of the pressure applies in each of the components of the eye, although the cornea is the first component which is being impacted and deformed, the sclera acts as a holder or container of the eye components to control the rupture/failure of the eye globe. In addition, due to the direct connection between the sclera and the optic nerve via the $\mathrm{ONH}$ site on one hand, and the essential role of the optic nerve in sending the information of the vision to the brain for the interpretation on the other hand, it is crucially needed to understand the sclera's mechanical properties.

\subsection{Sclera preparation, mechanical testing, digital image}

\section{correlation technique}

Thirty eye globes from fifteen human donors (age 42.25 \pm 7.14 ) (Mean \pm SD) were obtained from the Eye Bank of Iran (Tehran, Iran) under the permission from donors under the ethical rules of Legal Medicine Organization (LMO) based on the 2008 Declaration of Helsinki. Eyes were free of known diseases and the reason of death was not related to trauma according to the report of Medical Doctors with Legal Medicine Board. All the eyes were obtained within $7 \mathrm{~h}$ of 
death. The eyes were stored in a dried-stilled bottle which has an especial application for carrying the human organs for transplantation. They were not frozen at any stage as well. Thereafter, each eye was removed from the bottle and prepared by eradicating the inner part of the ocular structures to only have scleral shell. The fresh eye globe, the location of dissection and its process as well as the scleral shell are illustrated in Fig. 7. All dimension measurements were carried out using a digimatic ruler with a resolution of $0.005 \mathrm{~mm} \pm 0.05 \%$ (Insize, Vienna, Austria).

Then, the samples were mounted on the testing machine (Santam, Tehran/Hamburg, Germany) as presented in Fig. 8. The clamps were meanwhile lined with dry sandpapers to assure a no-slip boundary for the tissue samples. In addition to that, preload followed by eight cycles of preconditioning up to the elastic region of the tissue, which helped to dissipate the residual stress of the tissue, was applied to each sample before the test starts to ensure slip-free boundary condition of the tests (Fig. 9). Furthermore, in order to provide a condition as similar as possible to that of the human body, the mechanical tests were performed at the temperature and humidity of $37^{\circ} \mathrm{C}$ and $53 \%$ (Wall and Desk Hygrometers, W.W. Grainger, Inc, CA, United States), respectively. Then and there, tests were performed at eight different strain rates, including 5, 10, 20, $50,100,150,200$, and $500 \mathrm{~mm} / \mathrm{min}$. The reason why we chose these strain rates was that we aimed to have an understanding that how the sclera tissue will react to the force at different rates, ranging from the lowest up to almost the highest. 
Understanding of these mechanical properties under various rates of loading will help to have a better numerical eye model when it subjects to a blunt load at various rates. Because the rate of the applied load as a consequence of impact or any types of the injury may vary according to the speed of the impacted object. For example, changing the pressure of the aqueous body can induce a sever deformation (still in the linear range of the tissue properties) in other components of the eye according to the amount of IOP at a very short time span. Therefore, measurement of the mechanical properties of the sclera under various loading rates would provide suitable range of properties for injury simulations.

The tests were continued until the failure of the sclera tissue. Before the tests begin, the orientation of the collagen fibers in each of the sclera tissue histological images was determined. The images were taken via an optimized/customized optical microscope (Olympus DP73, Tokyo, Japan) as presented in Fig. 10.

The process was, before the uniaxial tensile test, the tissue samples were inserted into a $4 \%$ buffered formaldehyde solution $(\mathrm{pH} 7: 4)$ for fixation and further histological investigation. Specimens were tried to be maintained their planar geometry and sectioned serially at $5 \mu \mathrm{m}$ in circumferential orientation. In this way the fiber orientations in the $\left(\mathrm{x}_{1}, \mathrm{x}_{2}\right)$-plane could be seen on the histological images, where $\mathrm{x}_{1}$ and $\mathrm{x}_{2}$ express the circumferential and the axial directions as presented in Fig 11. A skilled histopathologist measured the orientation of at least 28 representative collagen fibers in the scleral intra wall per specimen from the histological images using Olympus Stream Image Analysis 
software (OSIA). This software can easily and accurately with less than $0.5 \%$ error, according to the device documentation, calculate the angle of each fiber component inside the histological images. Mean fiber angles and standard deviations were determined by assuming normal distribution and symmetrical arrangement with respect to the circumferential direction. The process of angle determination by the software was that, since the images took by the same software it could already know the dimension of the image. Thereafter, the images analyzed by the software of the microscope simultaneously. For example, an especial fiber in the sclera tissue wall was manually selected. The initial and end points of the fiber were then selected on the screen of the software. The software by itself drew a line between these two points. Then an imaginary $\mathrm{x}_{1}$ axis was lined parallel to the main $\mathrm{x}_{1}$ axis (Fig 11). The angle between the imaginary axis and the drawn line between the two points was then named as the fiber angle. All the 28 reprehensive collagen fibers in the same way were distinguished and calculated in one image. This process schematically is illustrated in Fig 11.

Thereafter, the stress relaxation test was conducted using the same device used for the tensile test. The stress relaxation test was started by measurement of the thickness, preload and preconditioning, ramp up to relaxation point, and recovery to the long term behavior of the tissue. The load in the tissue was recorded via a $50 \mathrm{kgf}$ load cell. Load was applied up to the elastic region of the tissue and kept for 1 minute. This also can be considered as a preload. Although samples were 
cut at a suitable angle to avoid or at least minimize residual stress, the preload and cyclic precondition load can also help the tissue to dissipate the residual stress for further analysis. Lastly, the sclera allows to be freely releases its energy of stress for about 60 minutes of relaxation and its stress-time diagram was recorded for the rest of the study. The time was decided according to the lively measurement of the tissue's behavior when a fully horizontal line is obtained. This process is presented in Fig. 12.

In this study in order to measure the strain/displacement of the tissues at each position, the video cameras with the capture rate of 280 frame/second and the resolution of $2048 \times 1088$ pixels were employed. The Simi Motion ${ }^{\circledR} 2 \mathrm{D} / 3 \mathrm{D}$ (Simi Reality Motion Systems GmbH, Max-Planck-Straße, Unterschleißheim, Germany) video camera software was also helped to lively measure the deformation of each marker in respect to the other ones as displayed in Fig. 13. Simi Motion has marker based automatic tracking to calculate marker positions. That is, the cameras focus on the tissue markers in a dark room. Thereafter, the measurement starts by starting the load applying. Since the testing machine was coupled with the measuring device system, by clicking the play both the load processing as well as the cameras start to record the measurements. The markers during the test on the wall of the sclera tissue relocated during applying the load as demonstrated in Fig. 14. Thereafter, the software of the video camera according to the location of the marker in the initial configuration and comparing 
it to its current configuration measures the displacement in the markers of the sclera tissue as presented in this figure.

In addition to this device, the testing machine was also benefitted from an extensometer. In order to compare the findings of these two methods, the stressstrain diagrams with the extensometer as well as the Digital Image Correlation (DIC) method is displayed in Fig. 15. The stress-strain diagrams among two approaches in this case in the initial section of the diagrams were totally the same. However, in the next section of the diagram is a bit different as the DIC data showed inconsiderably higher values. However, it should be pointed out in this case since the markers adhered to the sclera tissue wall, as a result, the measured displacement should be more accurate. In addition, In this way we can minimize the errors of the extensometer on the jaws of the machine at higher deformations.

\subsection{Linear elastic and nonlinear hyperelastic models}

The elastic modulus is the ratio of alteration in the stress values in respect to the strain and, therefore, stands for the strength of a material. It defined as the initial linear/Hookean section of the stress-strain diagrams of the tissue responses to the applied load. The hyperelastic material model was employed to address the nonlinear mechanical behavior of the sclera tissue. The experimental data was used to regulate the material coefficients of the hyperelastic Strain Energy Density Functions (SEDFs). Under the assumption that sclera tissue is an 
incompressible and isotropic material $[35,36]$, a general polynomial isotropic constitutive form is as following:

$W=\sum_{i+j=1}^{N} C_{i j}\left(\overline{I_{1}}-3\right)^{i}\left(\overline{I_{2}}-3\right)^{j}+\sum_{i=1}^{N} \frac{1}{D_{i}}(J-1)^{2 i} \vdots i, j=0,1, \ldots, N \vdots i+$

$j=1,2, \ldots, N$

$W$ is a function of two principal strain invariants only: $W=W\left(I_{1}, I_{2}\right)$, where $I_{1}$ and $I_{2}$ are defined as [37]:

$I_{1}=\lambda_{1}^{2}+\lambda_{2}^{2}+\lambda_{3}^{2}$

$I_{2}=\lambda_{1}^{2} \lambda_{2}^{2}+\lambda_{1}^{2} \lambda_{3}^{2}+\lambda_{2}^{2} \lambda_{3}^{2}$

Here, $\lambda_{1}^{2}, \lambda_{2}^{2}$, and $\lambda_{3}^{2}$ are the squares of the principal stretch ratios, linked by the relationship $\lambda_{1} \lambda_{2} \lambda_{3}=1$, due to incompressibility. $C_{i j}$ is the material coefficients which are determined after curve fitting to the experimental data. $\mathrm{D}$ is almost 0 for the incompressible materials. Then the $\mathrm{J}$ is introduced as the Jacobean matrix which equals to the determinant of the deformation gradient which in here due to incompressibility is 1 . This is according to the study which showed the Poisson's ratio values of $0.46-0.50$ indicating the incompressibility of the sclera tissue [38].

The principal axes of strain and stress are lined up with the direction of tension $\left(\mathrm{X}_{1}\right)$ and two orthogonal axes $\left(\mathrm{X}_{2}\right.$ and $\left.\mathrm{X}_{3}\right)$. Because of symmetry and incompressibility, the stretch ratios are defined as:

$\lambda_{1}=\lambda$ and $\lambda_{2}=\lambda_{3}=\frac{1}{\sqrt{\lambda}}$

where $\lambda \geq 1$ is the stretch ratio in the direction of tension. Moreover, Eqs. (2) and (3) give 
$I_{1}=\lambda^{2}+2 \lambda^{-1}$ and $I_{2}=\lambda^{-2}+2 \lambda$

$W$ just becomes the function of $\lambda$. During the experimental tension tests, the principal stretch ratio $\lambda$ was measured from the experimental strain $(e)$ by the following equation: $\lambda=1+e$. The stress is also calculated as the force/stress $\left(\mathrm{S}_{11}\right)$ tension per cross sectional area of the tissue measured by the load cell [39].

$S_{11}=\frac{d \widetilde{W}}{d \lambda}$, where $\widetilde{W}(\lambda) \equiv W\left(\lambda^{2}+2 \lambda^{-1}, \lambda^{-2}+2 \lambda\right)$

The Yeoh (Eq. (8)), Ogden (Eq. (9)), and Mooney-Rivlin (Eq. (10)) material models were then described as following:

$$
\begin{aligned}
& W=\sum_{i=1}^{3} C_{i 0}\left(\overline{I_{1}}-3\right)^{i}+\sum_{i=1}^{N} \frac{1}{D_{i}}(J-1)^{2 i} \\
& W=\sum_{i=1}^{N} \frac{\mu_{i}}{\alpha_{i}}\left({\overline{\lambda_{1}}}^{\alpha_{i}}+{\overline{\lambda_{2}}}^{\alpha_{i}}+{\overline{\lambda_{3}}}^{\alpha_{i}}-3\right)+\sum_{i=1}^{N} \frac{1}{D_{i}}(J-1)^{2 i} \\
& W=C_{10}\left(\overline{I_{1}}-3\right)+C_{01}\left(\overline{I_{2}}-3\right)+\frac{1}{D_{1}}(J-1)^{2}
\end{aligned}
$$

where $J=\operatorname{det}(F)$ and $\mathrm{F}$ is the deformation gradient. The terms, $\bar{I}_{1}$ and $\bar{I}_{2}$ are the first and second invariants, respectively, of the left Cauchy-Green strain tensor, B. For a normalized deformation gradient $\bar{F}=J^{-\frac{1}{3}} \bar{F}$ the Cauchy-Green strain tensor assumes the form: $B=\bar{F} \bar{F}^{T}$. The principal stretch, $\bar{\lambda}_{l}$ is the eigenvalue of $\bar{F}$ (deformation gradient). The polynomial coefficients, $\mu_{i}, \alpha_{i}$, and $C_{i j}$ and $a_{\mathrm{i}}$ are the material constants that were fit from the experimental data. As it was pointed out, the nonlinearity of the tissue was examined through the three different strain energy density functions, such as the Yeoh, Ogden, and Mooney-Rivlin, and their coefficients were also calculated thru the linear/nonlinear unconstrained 
minimization. The process goes as the experimental stress-strain diagram inputted into the programming code and, the employed SEDFs fitted to the experimental data. The parameters/coefficients in the material models were then determined and reported.

The material parameters of the hyperelastic models were numerically estimated via optimizing the stress-based nonlinear function

$f_{s}=\sum_{i=1}^{n}\left[\left(S_{11}=\frac{d \widetilde{W}}{d \lambda}\right)-S_{11}^{(i)}\right]^{2}$

where $n$ is the number of experimental data in the uniaxial tensile test. In this equations $S_{11}^{(i)}$ is the experimental data which we reported in the stress-strain diagram of the sclera tissue. The data in this regard is reported in Fig. 17. $\min f_{s}=\sum_{i=1}^{n}\left[\left(S_{11}=\frac{d \widetilde{W}}{d \lambda}\right)-S_{11}^{(i)}\right]^{2}<\delta$

where $\delta$ is a very small positive value. The objective is now to minimize the above $f_{s}$ function by considering all the material coefficients and other parameters. The process of minimization was carried out using the Matlab optimization package.

In order to figure out whether the experimental boundary conditions as well as the loads are validate enough or not, a comparative study between the experimental results and that of the numerical ones through a FE model of the uniaxial tensile setup along with the sclera tissue were carried out. According to the size of the scleral tissues, the FE model was made with the length, width, and thickness of 20,13, and $1.5 \mathrm{~mm}$, respectively. The experimental stress-strain data (the ones calculated in the current study) at two different strain rates were then 
assigned to the model. That is, the experimental curves as data points were inputted as the material properties of the sclera tissue in the FE code. Therefore, the sclera tissue in the model was behaved according to the experimental data. Since the model was made according to the experimental data, it was aimed to investigate the outcome of its response to the applied load and boundary, and then compare the findings. The results were then compared to the experimental as well as the SEDFs data. Hence, a model was employed and the stress-time along with the strain-time results at the tracked nodes were recorded. Then, by the application of 'combine' algorithm in a commercial software, the stress-strain diagrams were plotted. However, it should be noted that due to the long simulation time, only the samples at the strain rates of 5 and $100 \mathrm{~mm} / \mathrm{min}$ were simulated and compared. In addition to that, the agreement of the SEDFs in comparison to the experimental data was determined using the unconstrained linear/nonlinear minimization and the obtained results are plotted.

\subsection{Viscoelastic model}

The stress-relaxation test was performed to calculate the viscoelastic coefficients of this tissue via a Prony series. To do this, six samples were subjected to a 1 minute holding load and then let release their energy up to 60 minutes. The Prony series was employed is presented as following: $G(t)=G_{\infty}+\sum_{i=1}^{3} G_{i} e^{-\beta t}$ 
where $G_{\infty}$ is the long term relaxation coefficient $\left(G_{\infty}=\lim _{t \rightarrow \infty} G(t)\right)$ and $G_{i}$ coefficients show the relaxation strength corresponding to the $\beta$ decay constant $[40,41]$. A compressive quasi-static load up to $60 \%$ of the sclera length was applied to the sclera and, then, it lets release its energy for up to 60 minutes. The viscoelastic coefficients were calculated from the hold region of the reduced relaxation function-time diagram via MATLAB v. R2014a (The MathWorks, Inc., Natick, MA, the United States). The fit was verified using the averaged coefficient of a determination $\left(R^{2}\right)$.

The process of linear viscoelastic calculation from the stress-strain as well as stress-time of the tissue under the stress-relaxation load is presented in Fig. 16.

\subsection{Statistical analysis}

Analyses were based upon data values that were expressed as Mean \pm SD. Data from the tissues at different strain rates were compared using post hoc with the Scheffe method [42] with a significance of $p<0.05$.

\subsection{Results}

The mean stress-strain diagrams of the sclera tissues at various strain rates, i.e., 5, 10, 20, 50, 100, 150, 200, and $500 \mathrm{~mm} / \mathrm{min}$, are indicated in Fig. 17. The load direction was set to the circumferential direction of the tissue since it is known that the mean angle of collagen fibers is tend to be aligned in that direction 
[43]. Each curve is the representative of three samples at a specific strain rate. The stress-strain diagrams show the nonlinear behavior of the sclera tissue since it is considered as a soft connective biological tissue. The tissue results revealed that almost all the samples, regardless of the strain rates, failed at a similar strain value (50-65\%). This suggests the insignificant role of the strain rate on the strain failure of the sclera tissue. The load in the samples was applied in the circumferential direction of the tissues which helps to be able to measure the stiffest mechanical response of the sclera tissue under various strain rates. Considering the stress-strain diagrams, the elastic modulus as well as the maximum stress of the sclera tissues at eight different strain rates were calculated and plotted in Fig. 18. The results exhibited the highest amount of elastic modulus and maximum stress at the strain rate of $200 \mathrm{~mm} / \mathrm{min}$ with 2.93 and $2.91 \mathrm{MPa}$, while the lowest ones were observed at the strain rate of $5 \mathrm{~mm} / \mathrm{min}$ with 1.10 and 0.95 MPa. The scatter of the results showed the strain rate dependency of the sclera tissue which was predictable owing to the collagen content of this tissue.

That is, the elastic modulus and maximum stress of the tissue at the strain rate of $200 \mathrm{~mm} / \mathrm{min}$ were 2.66 and 3.06 times higher than that at the strain rate of the $5 \mathrm{~mm} / \mathrm{min}(p<0.05$, post hoc Scheffe method).

The nonlinear stress-strain behavior of the scleral tissues under higher amount of strain (0.15> ) implies that considering the sclera as a simple elastic material is not enough. Therefore, three strain energy density functions, including the Yeoh, Ogden, and Mooney-Rivlin, were examined to address the nonlinear 
mechanical behavior of the sclera tissue. In addition to that, a FE model of the sclera tissue during load bearing was established to compare the experimental as well as the constitutive findings with that of the numerical ones as presented in the inset of Fig. 19a. The results for the strain rate of 5 and $100 \mathrm{~mm} / \mathrm{min}$ are reported in Figs. 19a and 19b. To sum up, the data in these figures are showing the experimental stress-strain diagram, FE data which was extracted from the model that followed the experimental stress-strain diagram, and the SEDFs curves which were plotted according to the experimental data using nonlinear/linear unconstrained minimization algorithm. The FE results in here showed that the boundary conditions as well as the applied load in the experimental section can be well verified via the employed FE model. That is, the FE modeling results revealed that the nodes in the middle of the FE tissue model can illustrate a set of consistent stress-strain data points which are found to be in good agreement with that of the experimental ones. The hyperelastic material coefficients of the sclera tissue and summarized in Table 2.

The stress-strain as well as the elastic modulus and maximum stress data of the scleral tissues at various strain rates revealed that the mechanical properties of the sclera tissue are highly affected by the strain rate. That is, the mechanical properties of the sclera tissue can be varied at various rates of load, and this implies the viscoelastic behavior of the sclera tissue. The stress-time of the samples were then quantified and reported in Fig. 20a. The normalized reduced relaxation function was also calculated and illustrated in Fig. 20b. The 
viscoelastic coefficients were computed using MATLAB code and depicted in

Table 3.

\subsection{Discussions}

The material/mechanical properties of the sclera which is the outer coats of the eye are of increasing interest to some scientists as well as clinicians. It is because the mechanical properties of the sclera progress the surgical techniques that affect by the material/mechanical properties of this delicate soft material [44]. In addition, understanding the mechanical properties of the sclera in here would help to establish a more suitable eye model to be used for further accurate numerical simulations.

The stress-strain diagrams of the sclera well approved the nonlinear mechanical behavior of this tissue (Fig. 17) as it has been well approved by the previous studies $[45,46]$. Since the load direction was aligned in the circumferential direction which is actually so close to the mean angle of collagen fibers, it was expected to have stiffer mechanical properties of the sclera. The elastic modulus and maximum stress values were then calculated from the linear Hookean regions and end of the stress-strain curves, respectively (Fig. 17). The highest elastic modulus as well as the maximum stress were observed at the strain rate of $200 \mathrm{~mm} / \mathrm{min}$ with 2.92 and $2.91 \mathrm{MPa}$, respectively, while the lowest ones were seen at the strain rate of $5 \mathrm{~mm} / \mathrm{min}$ with 1.10 and $0.95 \mathrm{MPa}$, respectively. The results also showed strong strain rate dependency of the scleral tissue in a 
way that, for instance, the elastic modulus and maximum stress at the strain rate of $500 \mathrm{~mm} / \mathrm{min}$ are 2.48 and 2.12 times higher than that of the $5 \mathrm{~mm} / \mathrm{min}$. No meaningful relation between the rates of the strain and the values of elastic modulus and maximum stress was detected since these values at the strain rate of $200 \mathrm{~mm} / \mathrm{min}$, for example, is lower than that of the $500 \mathrm{~mm} / \mathrm{min}$ (Fig. 18). Similarly, the elastic modulus and maximum stress of the sclera tissue at the strain rate of 20 and $50 \mathrm{~mm} / \mathrm{min}$ were higher than that of the $100 \mathrm{~mm} / \mathrm{min}$. It has been reported that the sclera's elastic modulus varies between $1.8 \pm 1.1 \mathrm{MPa}$ for the posterior and 2.9 $\pm 1.4 \mathrm{MPa}$ for the anterior sides [16]. This is in good agreement with our experimental results since they vary between 1.10 to $2.92 \mathrm{MPa}$. The nonlinearity of this tissue was examined through three different SEDFs, such as the Yeoh, Ogden, and Mooney-Rivlin, and their coefficients were also calculated thru the nonlinear/linear unconstrained minimization (Table 2). The results revealed the ability of the Yeoh, Ogden, and Mooney-Rivlin material models to address the mechanical response of the tissue. Each material model has its own advantage in modeling the soft biological tissues. The Ogden material model is expressed in terms of the principal stretches while the Mooney-Rivlin is defined as a function of the first and second invariant of the unimodular component of the left Cauchy-Green deformation tensor. However, the Yeoh material model is just based on the first invariant of the deformation tensor and this is why it is called the reduced polynomial model. Since very soft tissues, such as the brain $[47,48]$, has a profound nonlinear response to the mechanical load, Ogden material model 
is highly common. On the other hand, the materials such as arterial tissues [49] can fit with Mooney-Rivlin material models. This is the reason why this study three different material models were examined. Furthermore, the numerical FE data well approved the ability of the Mooney-Rivlin material model to define sclera's mechanical behavior. The reason is that Mooney-Rivlin material model enables to consider the role of the function of the first and second invariant of the left Cauchy-Green deformation tensor which in this study because the load was applied in the stiffest direction of the sclera tissue, this material model enables to have a better presentation of the tissue response. However, the Ogden model was based on the principal stretches which were failed to have a tangible understanding of tissue response. Similarly, since the Yeoh material model was just based on the first invariant of the deformation tensor, its results could not capture the nonlinear stiff mechanical response of the tissue. Due to a very long simulation time, only two samples, one of the low range $(5 \mathrm{~mm} / \mathrm{min})$ and one from the high range $(100 \mathrm{~mm} / \mathrm{min})$ strain rates were chosen and simulated. Although in the case of $5 \mathrm{~mm} / \mathrm{min}$, the FE data as well as the Ogden data were diverged from the experimental data, in almost all the locations of the curve the results are in good agreement with each other (Fig. 19).

The results showed that a significant amount of energy of the sclera was dissipated within 60 minutes (Fig. 20). That is, the initial stress of the sclera was started with $0.7 \mathrm{MPa}$, then it continues to decrease by the value of $\left(\mathrm{G}_{1}+\mathrm{G}_{2}+\mathrm{G}_{3}\right) \mathrm{e}^{-}$ ${ }^{\beta}$, and finally reached to $0.03 \mathrm{MPa}$ after an hour which is defined as the long-time 
shear modulus. This implies the time-dependency mechanical behavior of the sclera tissue (Table 3). The summation of the exponential factor in here must be 1 to show the accuracy of the fitting approach.

Different material models would have different outcomes in the FE models since the deformation of the materials are strongly related to their stiffness/compliance. In a FE model according to the objective of a modeling, different material models can be employed. As the elastic and hyperelastic material models are almost using for the small and large deformations, respectively, employing them in the designated models are preferable. However, since the sclera presented a time-dependent mechanical behavior under the applied load, the application of viscoelastic model could be reasonable. Therefore, in the impact simulations, which there is a very short simulation time, the application of the viscoelastic material model might not be accurate enough to be considered. The experimental histological analysis of the present study (Fig. 17 inset) revealed that the mean angle of collagen fibers is $63.86 \pm 4.12$ $(\mathrm{Mean} \pm \mathrm{SD})$ degree in respect to the longitudinal axis. It means that the collagen fibers in the scleral tissue are mostly aligned in the circumferential direction, and this can lead to higher/stiffer mechanical properties for the sclera tissue in this direction. As it was pointed out, the mechanical properties of the sclera out of this section was assigned to the eye model proposed in the next chapter under various strain rates. Having the mechanical properties of the sclera tissue under various rates also helped to be able to simulate the penetrating on a basis of impact rate. 
In addition, as it was pointed out, the mechanical properties of the sclera have key asset in $\mathrm{ONH}$ biomechanics as well as visual acuity, and generally eye performance. 
Table 2. Hyperelastic material coefficients of the sclera at various strain rates.

\begin{tabular}{lccccc}
\hline Mooney-Rivlin & $C_{10}$ & $C_{01}$ & $C_{20}$ & $C_{11}$ & $C_{02}$ \\
\hline 5 & 0.255 & -0.234 & -1.046 & 0.569 & 1.727 \\
10 & 3.019 & -2.886 & -1.474 & 5.352 & -5.934 \\
20 & 0.509 & -0.465 & 0.925 & -3.150 & 2.794 \\
50 & 4.016 & -4.110 & -1.751 & 5.719 & -5.946 \\
100 & 0.152 & -0.151 & -1.015 & 2.706 & -1.392 \\
150 & 4.856 & -5.007 & -2.979 & 9.915 & -9.968 \\
200 & 4.185 & -4.232 & -1.329 & 5.118 & -6.218 \\
500 & -0.886 & 0.941 & 2.112 & -7.480 & 7.312 \\
\hline Ogden & $\mu_{1}$ & $\mu_{2}$ & $\alpha_{1}$ & $\alpha_{2}$ & - \\
\hline 5 & 11.457 & -11.441 & 1.993 & 1.387 & - \\
10 & 1.626 & -1.244 & 1.795 & -6.932 & - \\
20 & 16.139 & -16.087 & 0.007 & -0.492 & - \\
50 & 1.869 & -2.081 & 5.084 & -12.078 & - \\
100 & 7.888 & -7.878 & 2.356 & 1.916 & - \\
150 & 1.873 & -2.066 & 4.228 & -10.295 & - \\
200 & 1.634 & -1.523 & 3.178 & -7.641 & - \\
500 & 21.505 & -21.604 & -0.085 & -0.509 & - \\
\hline Yeoh & $C_{10}$ & $C_{20}$ & $C_{30}$ & - & - \\
\hline 5 & 0.0317 & 1.019 & -1.034 & - & - \\
10 & 0.274 & 0.122 & -0.076 & - & - \\
20 & 0.088 & 0.276 & -0.084 & - & - \\
50 & 0.102 & 0.697 & -0.353 & - & - \\
100 & -0.004 & 0.345 & -0.105 & - & - \\
150 & 0.089 & 0.545 & -0.244 & - & - \\
200 & 0.173 & 0.217 & -0.046 & - & - \\
500 & 0.039 & 0.315 & -0.096 & - & - \\
\hline & & & & &
\end{tabular}


Table 3. Viscoelastic parameters of the sclera.

\begin{tabular}{lc}
\hline Material constants & \\
\hline $\mathrm{G}_{\infty}(\mathrm{MPa})$ & $0.03607 \pm 0.0004$ \\
$\mathrm{G}_{1}$ & $0.1422 \pm 0.0012$ \\
$\mathrm{G}_{2}$ & $0.1646 \pm 0.0016$ \\
$\mathrm{G}_{3}$ & $0.7125 \pm 0.0029$ \\
$\beta$ & $0.7325 \pm 0.0022$ \\
$R^{2}$ & 0.9563 \\
\hline
\end{tabular}




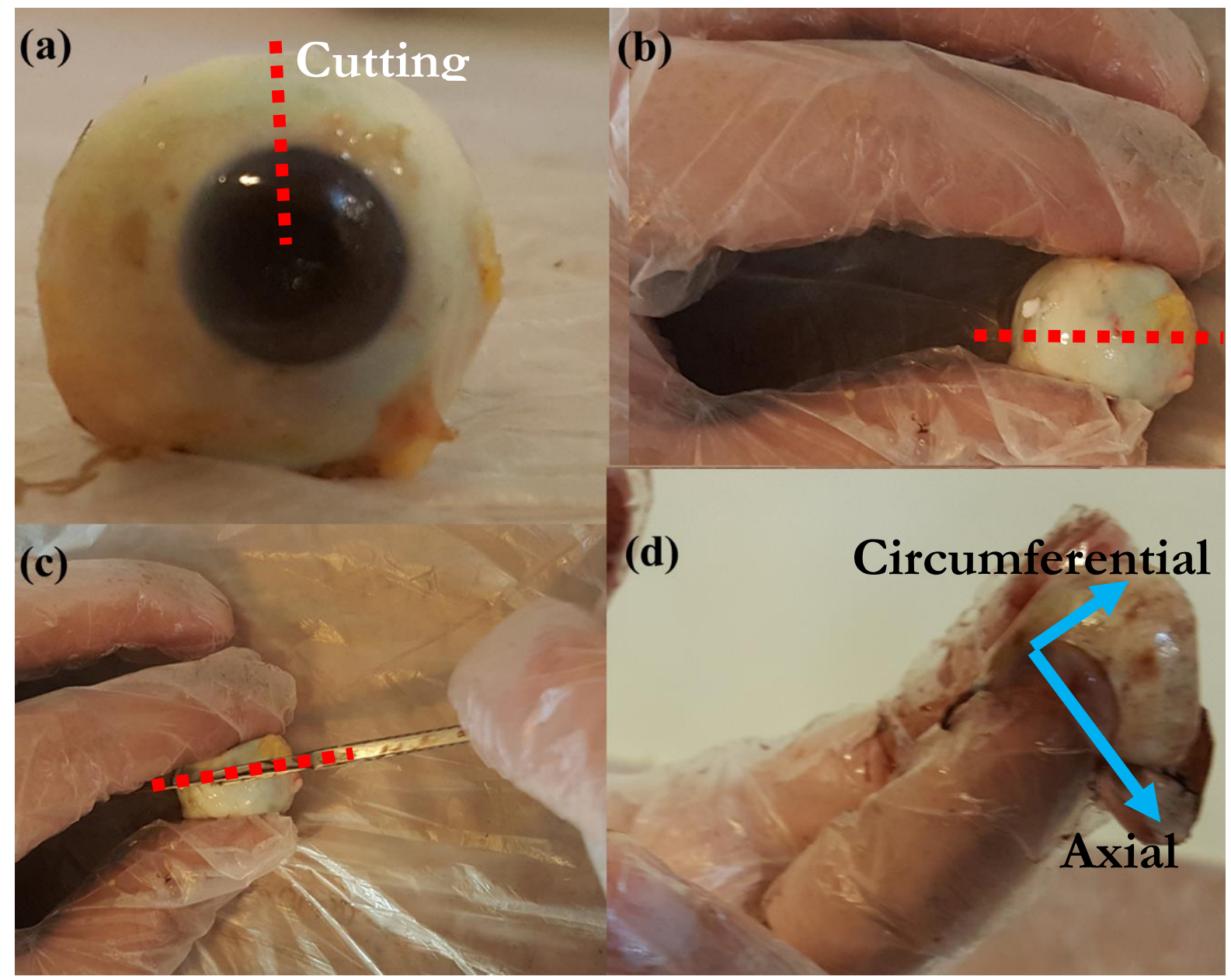

Fig. 7. (a) The fresh eye globe, (b and c) the process of globe dissection, and (d) final sclera shell. The axis in here shows the cut line. 


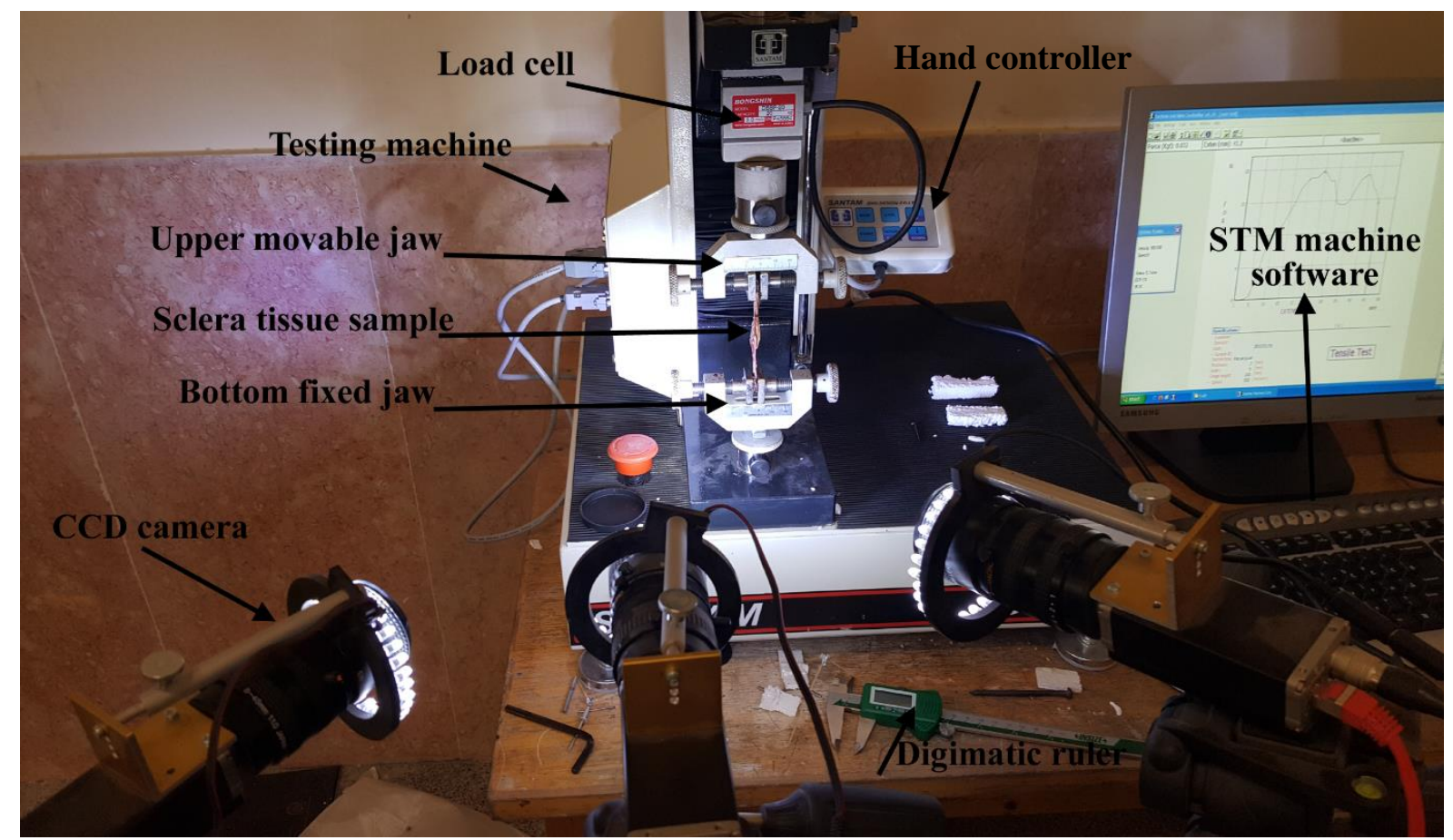

Fig. 8. The sclera tissue under the applied load. The upper jaw of the testing machine is moved at various strain rates while the lower jaw is fixed. The load cell as well as extensometer/DIC technique measured the force and displacement of the sclera tissue, respectively. The load-displacement curves lively appeared on the screen of the device and recorded for further mechanical analyses. In addition, three CCD cameras (280 frame/sec) helped us to have an accurate measurement on the detail of deformation in the tissue wall. The digimatic ruler in here was also used for the initial measurement of the jaws distance, tissue thickness, etc. 


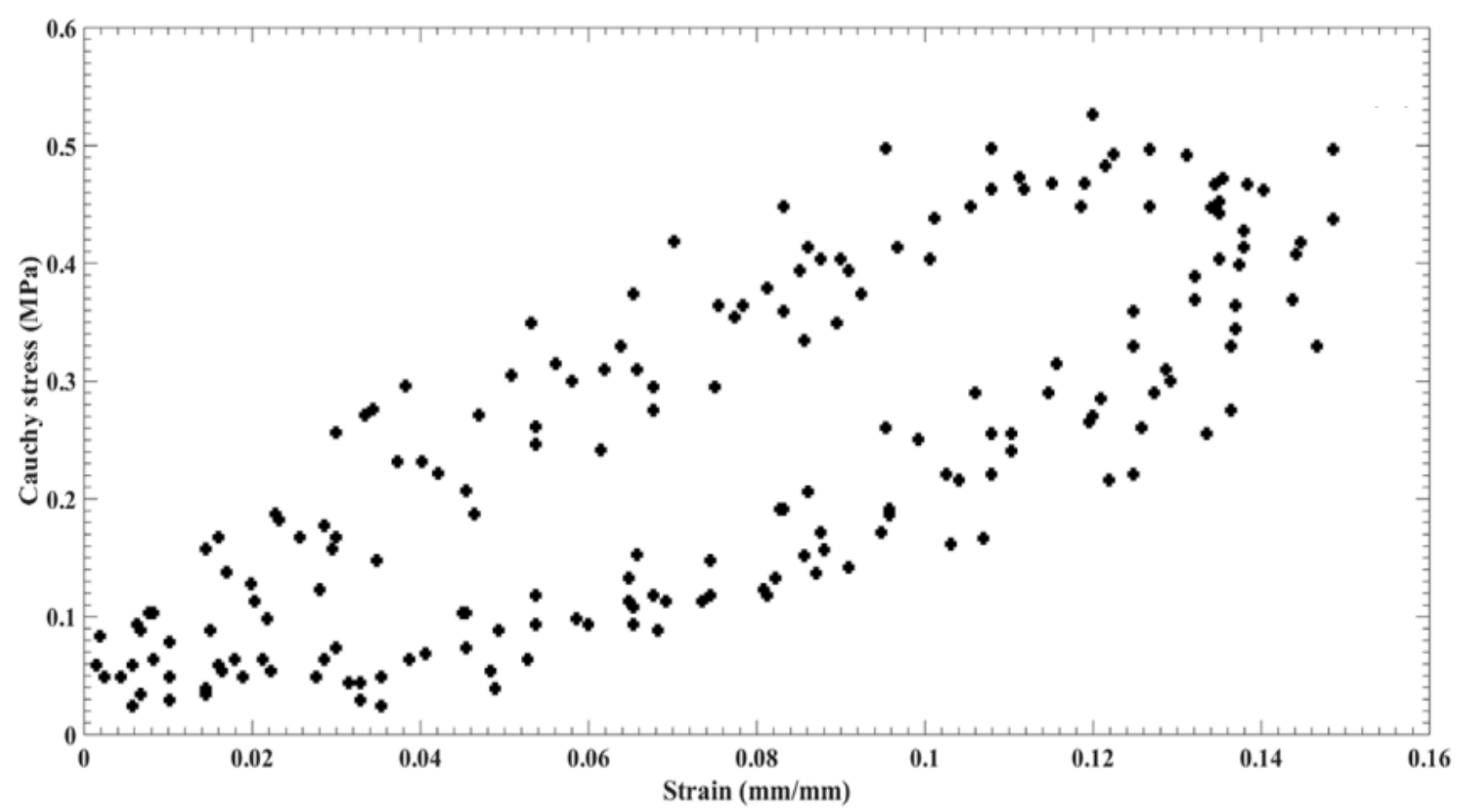

Fig. 9. The preconditioning of the sclera tissue was applied up to the elastic region of the tissues. 


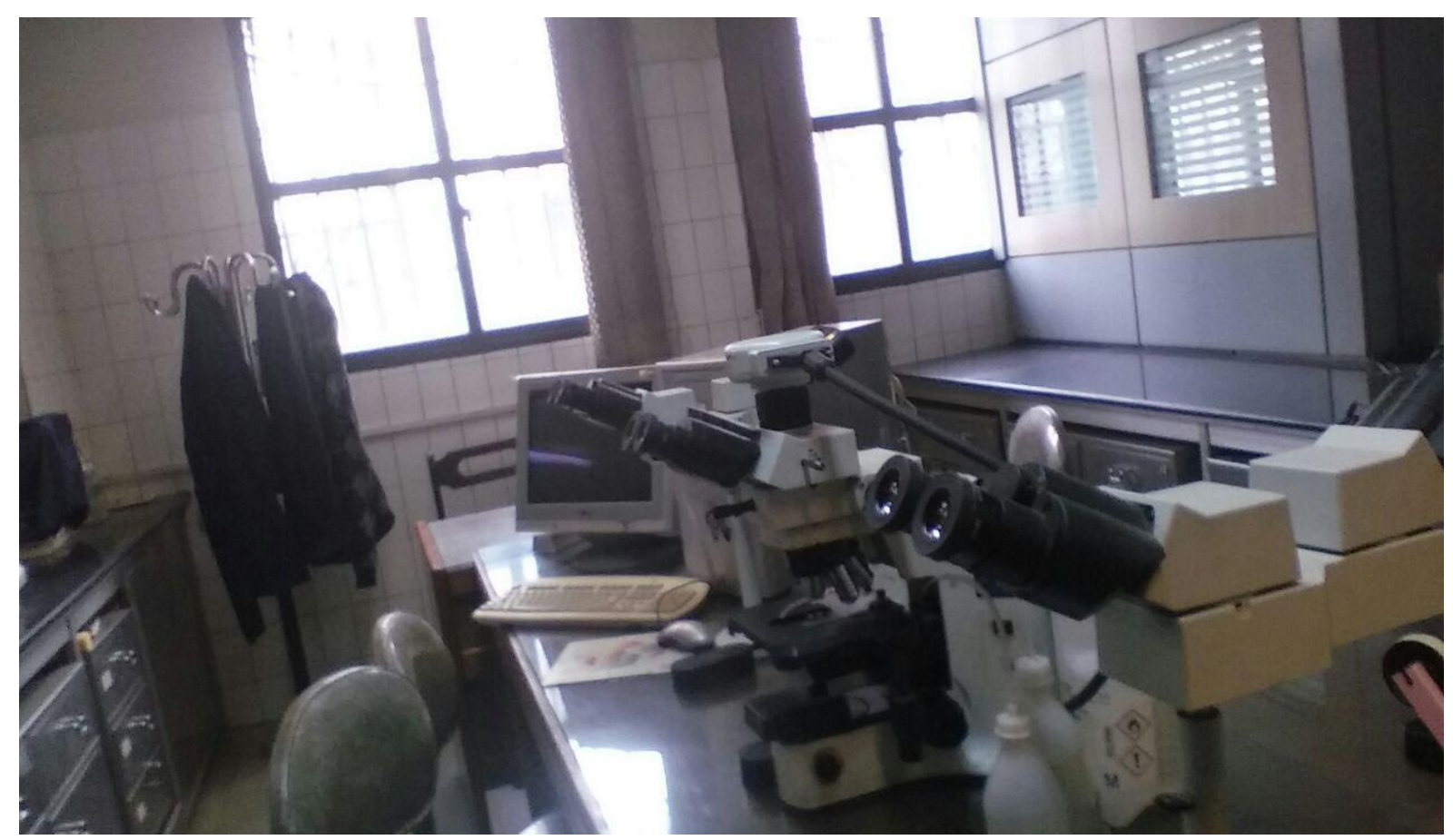

Fig. 10. The microscope and its CCD camera set used for the histological analyses.

The Olympus Stream Image Analysis Software (OSIAS) was also used to measure the fiber angles. 


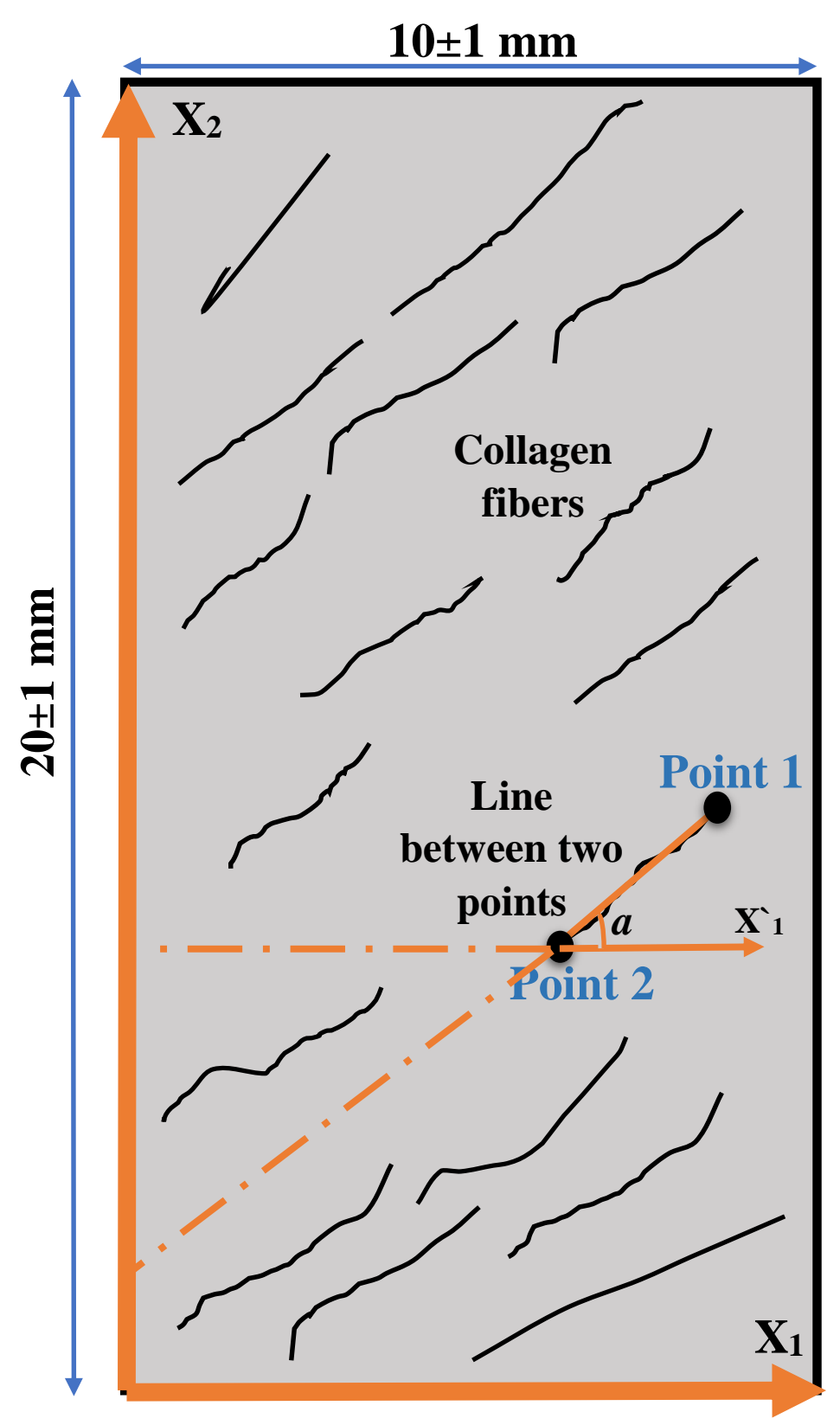

Fig. 11. The histological image was analyzed using the OSIA software. An specific fiber was selected on the tissue wall. Manually the initial and end points of the fibers were selected. The software automatically draws a line between these two points and also provides an imaginary axis parallel to the main axis. The angle between these the line and the axis was calculated as the $a$ and reported. Whole process carried out by an expert histopathologist. 


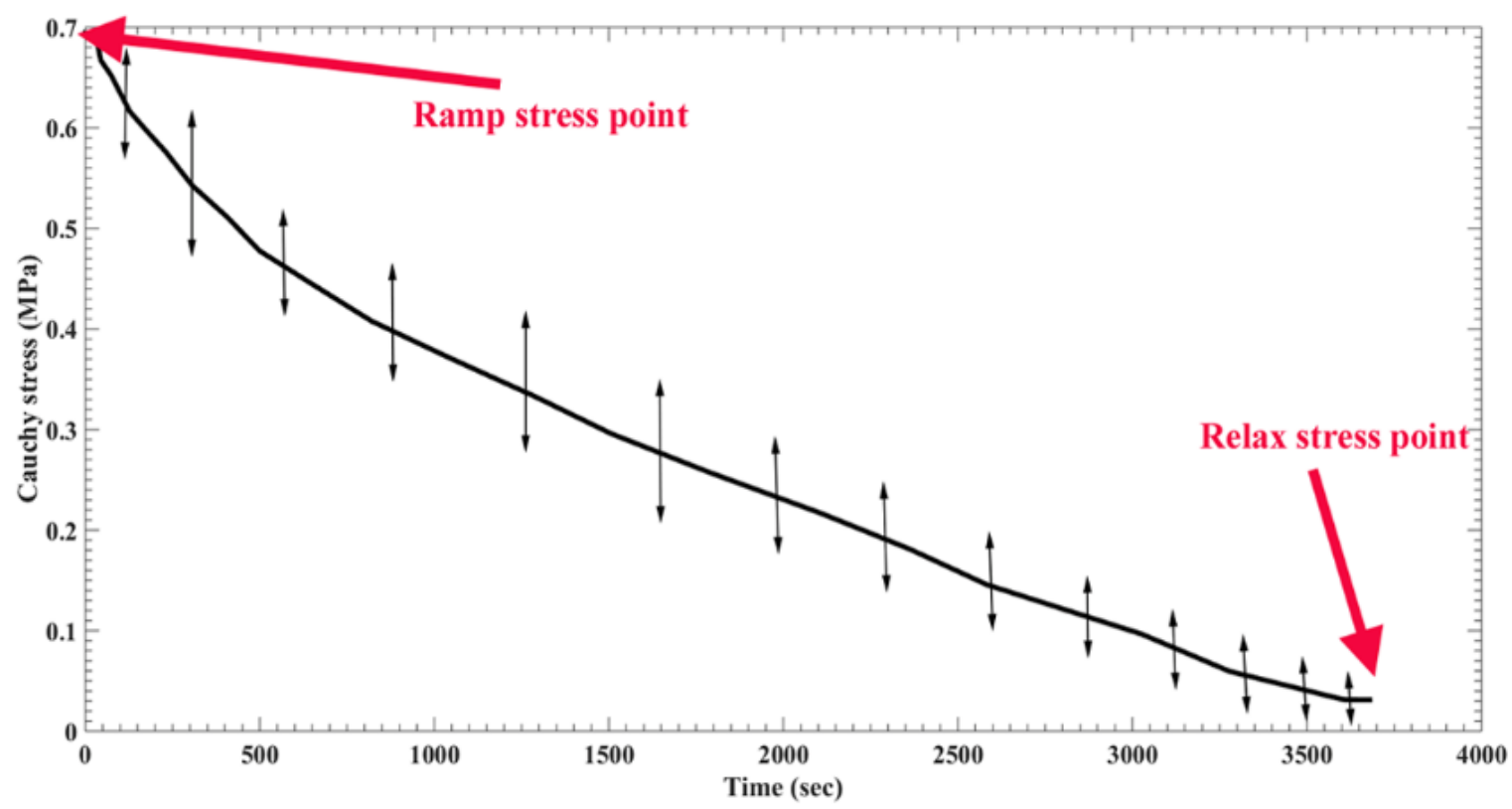

Fig. 12. The ramp, relaxation, and recovery. The load was applied on the tissue and allowed to be released for 60 minutes. The time of releasing was chosen according to the least amount of difference compared to the horizontal line in the tissue. 


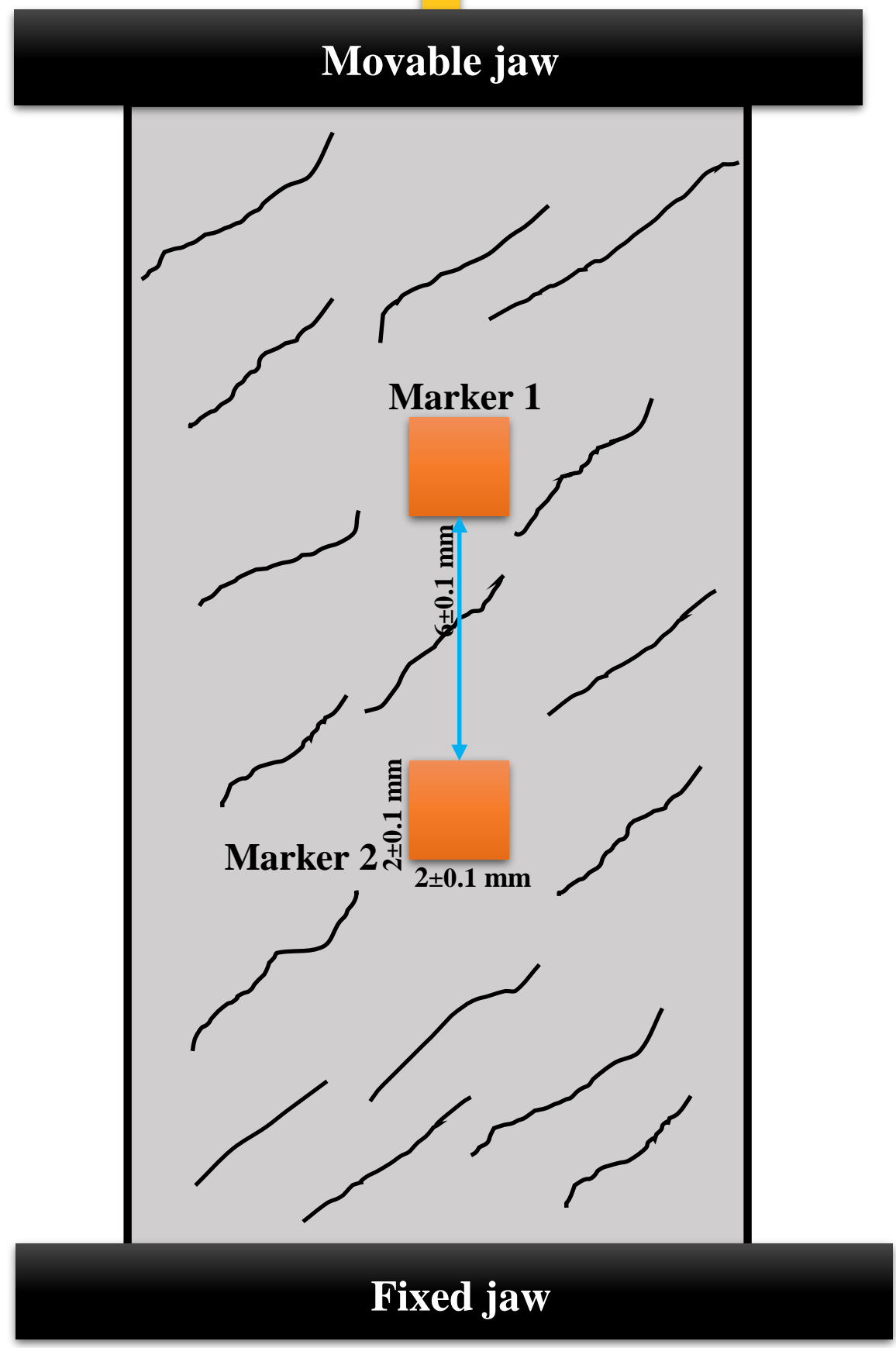

Fig. 13. The schematic of the sclera tissue under the applied load. The markers were placed on the tissue wall and their relocation comparing to their initial distance were measured lively. 


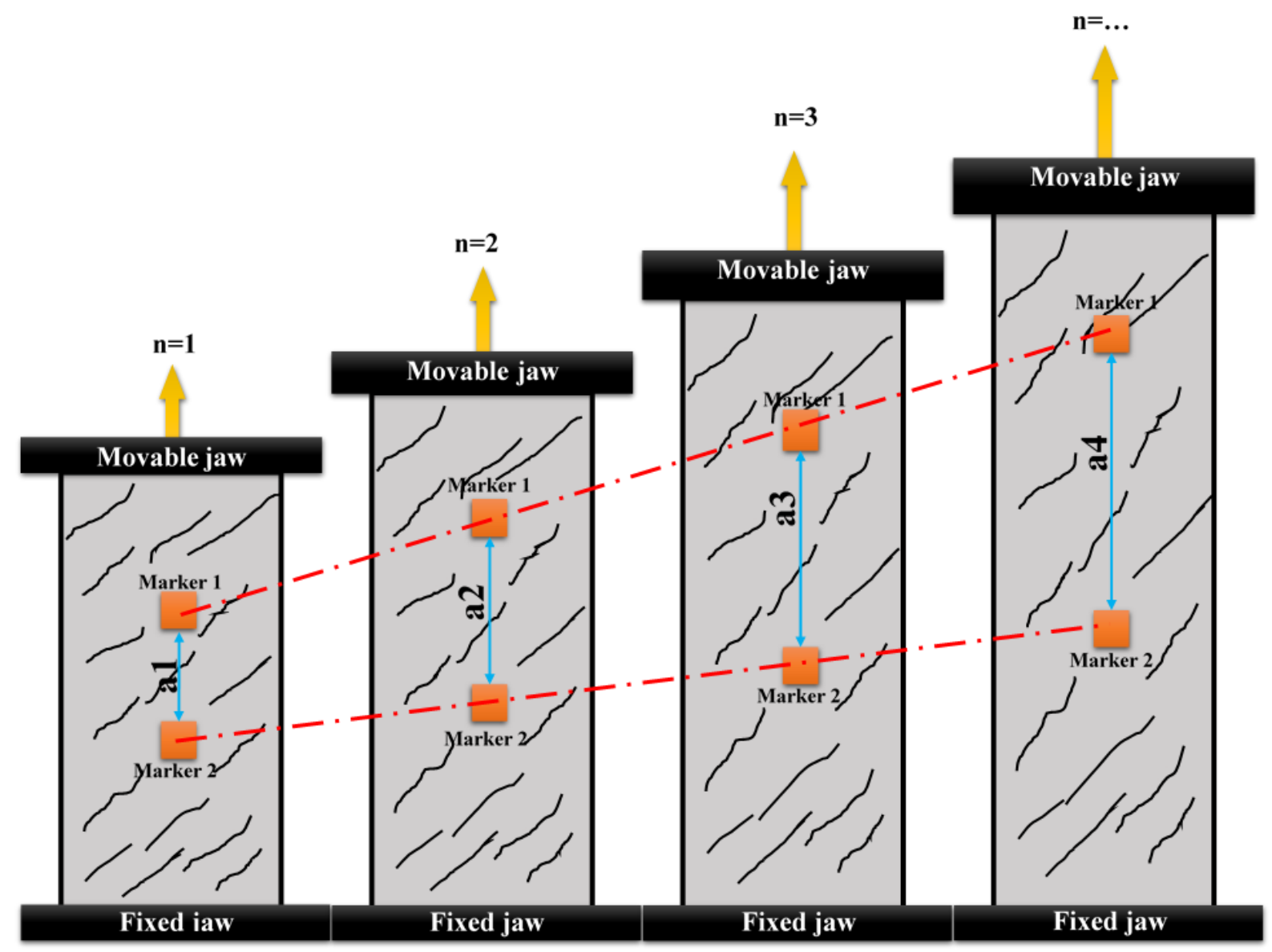

Fig. 14. The schematic of the marker's movement on the tissue wall. The ' $n=1$ ' is the initial stage and this stage can continue up to the tissue failure point $\left(\mathrm{n}_{\text {final }}\right)$. The ' $a$ ' stands for the distance between the markers which is changing during load applying on the tissue wall. The $\mathrm{a}_{1}$ is the initial distance while the $\mathrm{a}_{\text {final }}$ is the distance between the markers at the final point. 


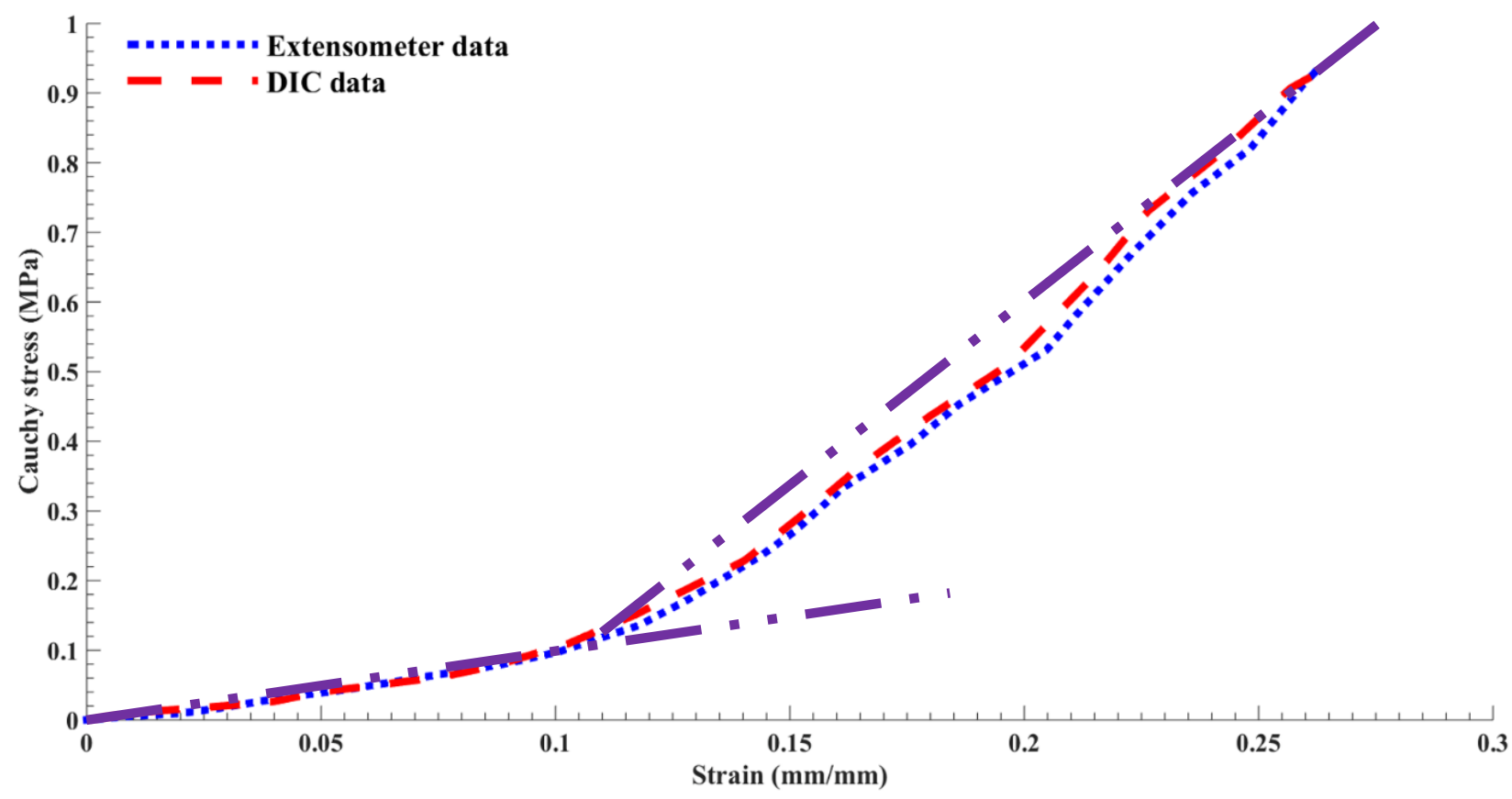

Fig. 15. The comparative stress-strain diagrams between the extensometer and DIC methods. 


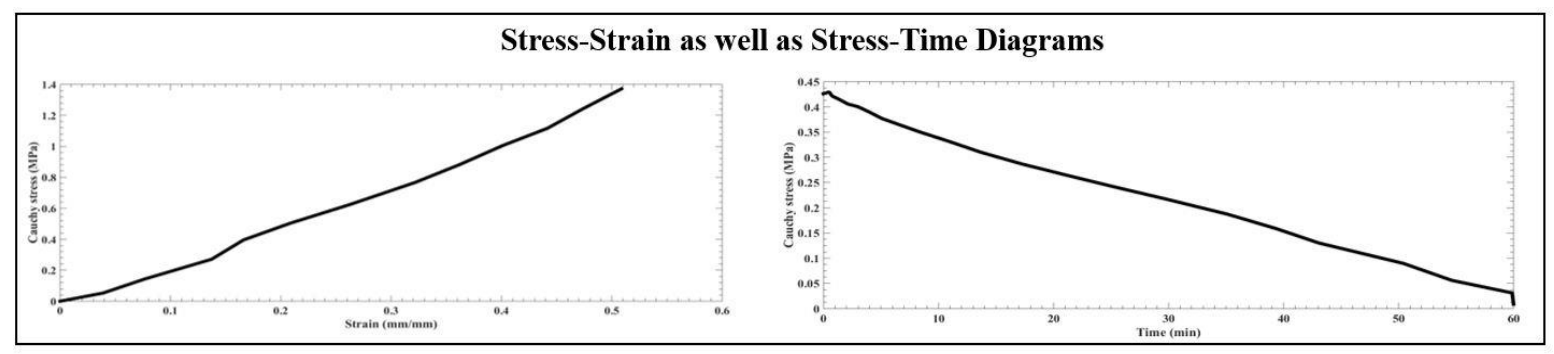

The amount of elastic modulus in the stress-strain data was measured by stress/strain value. The amount of $G=E / 2(1+v)$. Thereafter, the amount of G-Time was plotted.

In order to calculate the coefficients of the reduced relaxation function, the normalized curve of the G-Time was plotted. Using the normalized G-Time, the coefficients using the simple curve fitting were calculated.
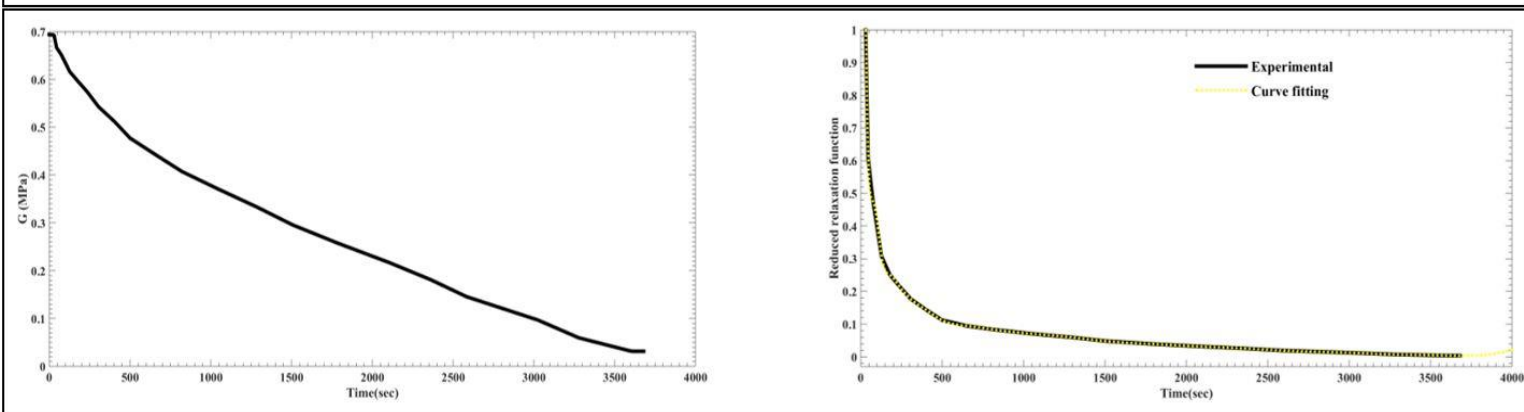

Fig. 16. The process of linear viscoelastic calculation from the stress-strain as

well as stress-time of the tissue under the stress-relaxation load. 


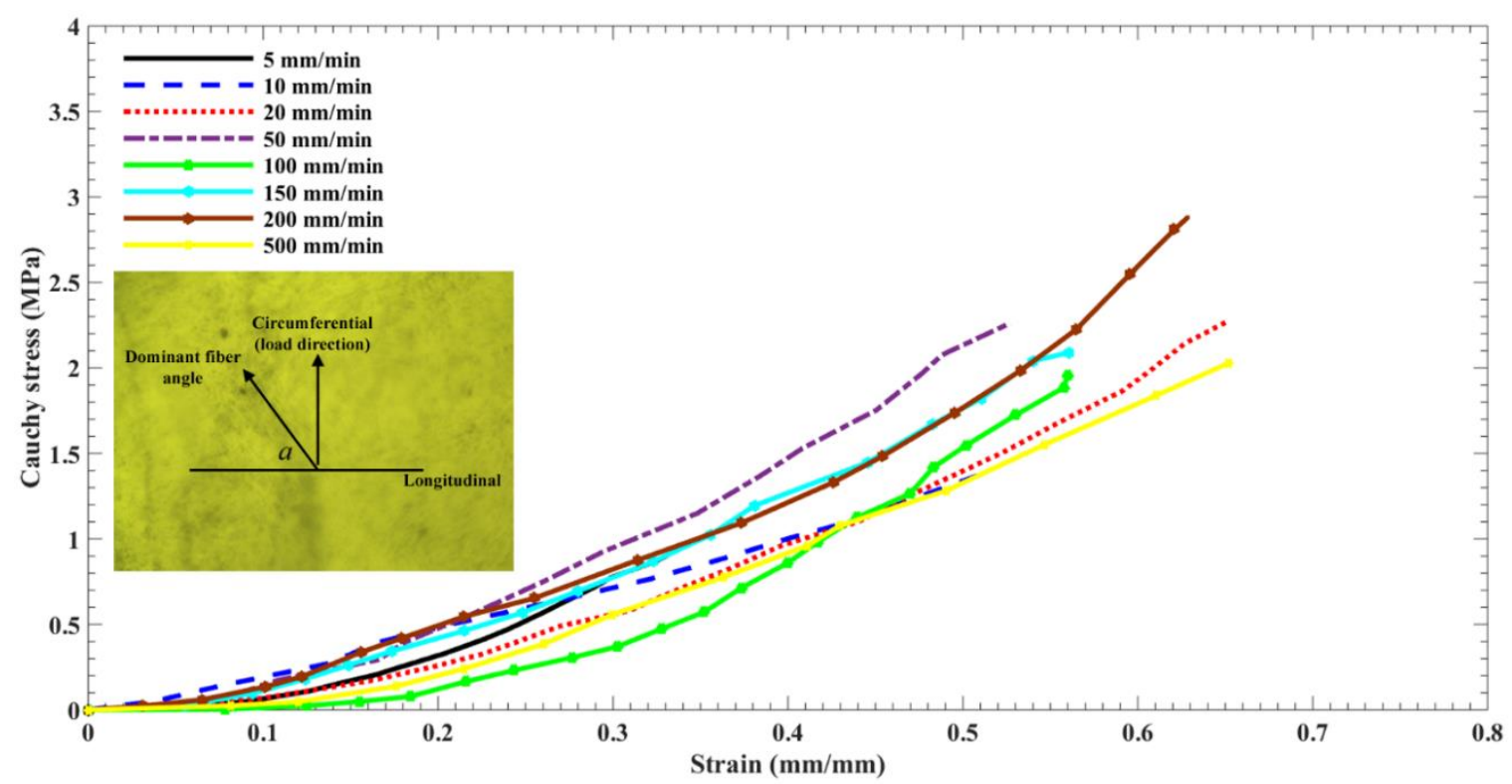

Fig. 17. The mean stress-strain diagrams (each curve is the average of three different sample curves) of the sclera tissue under various strain rates. Moreover, the histological image of the sclera (the inset of the figure) before applying the load is demonstrated. 


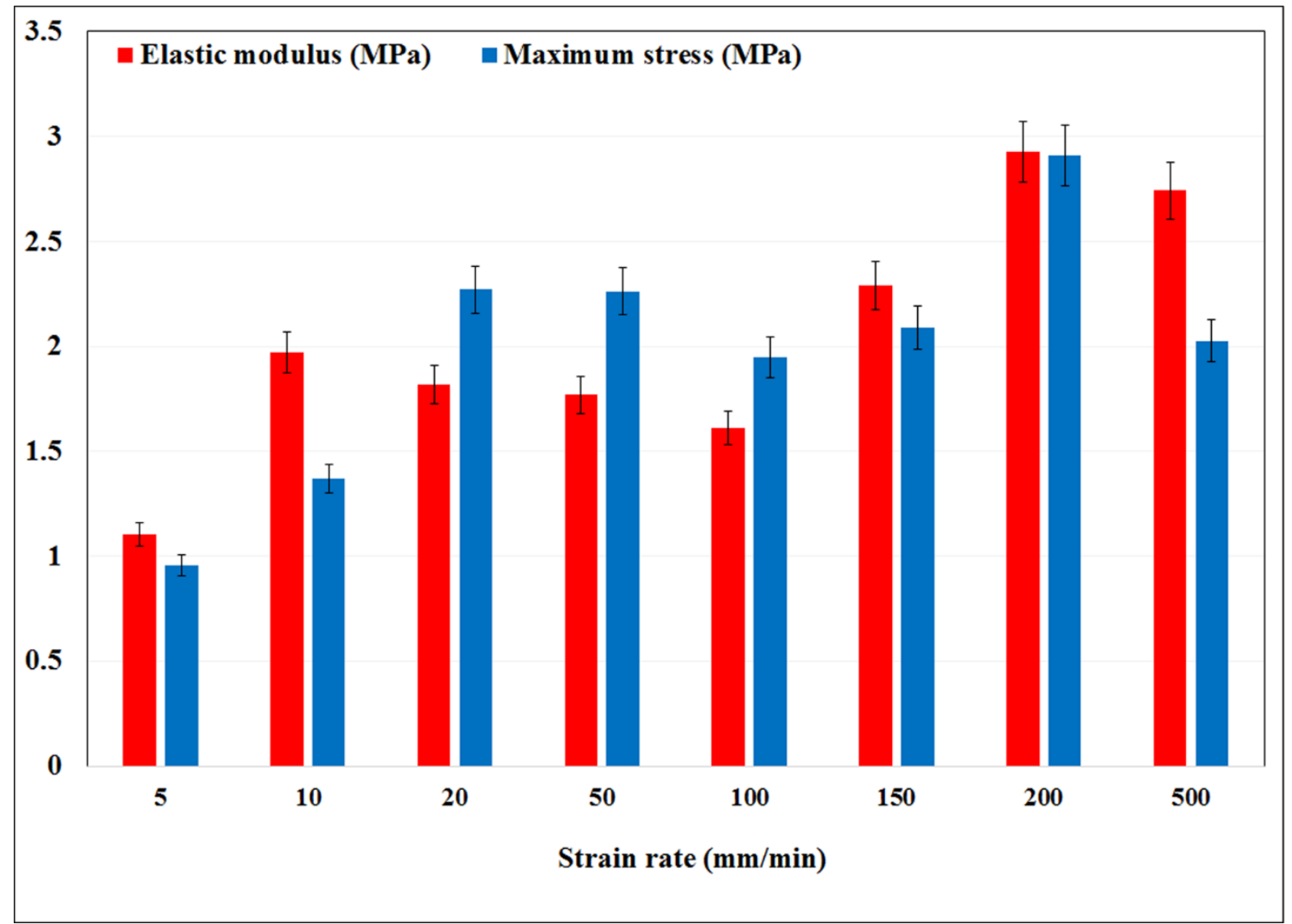

Fig. 18. A comparative bar chart of the elastic modulus as well as the maximum stress of the sclera tissue at various strain rates. The amount of elastic modulus and maximum stress at the rate of $200 \mathrm{~mm} / \mathrm{min}$ are significantly higher than that of the other rates of loading $(p<0.05)$. In addition, these amount at the strain rates of the $10,20,50,100$, and $150 \mathrm{~mm} / \mathrm{min}$ are higher than that of the $5 \mathrm{~mm} / \mathrm{min}$. The amount of elastic modulus at the strain rate of $200 \mathrm{~mm} / \mathrm{min}$ is not significantly higher $(p>0.05)$ than that of the $500 \mathrm{~mm} / \mathrm{min}$ while the amount of maximum stress is significantly higher $(p<0.05)$. 

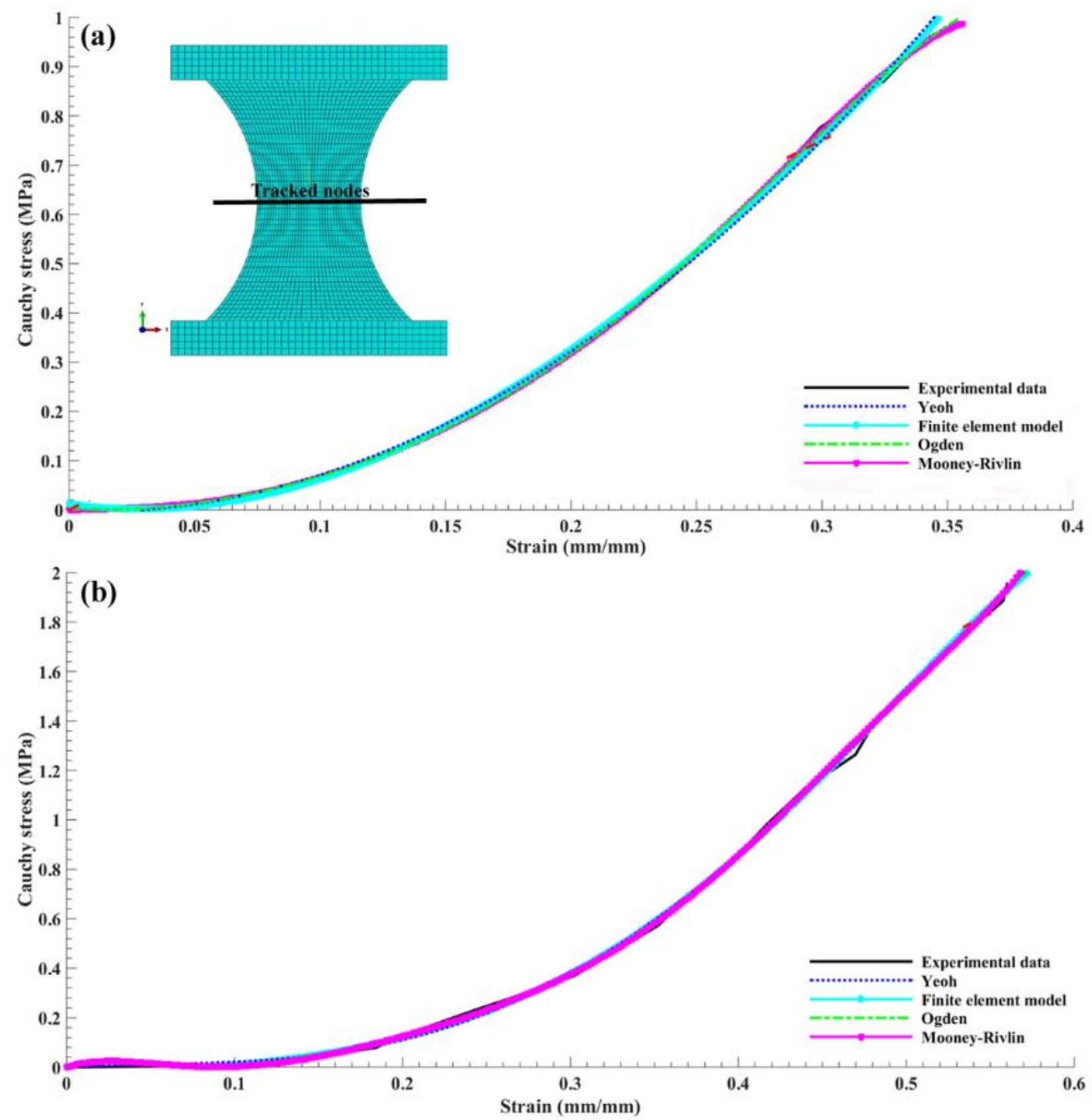

Fig. 19. The uniaxial stress-strain responses of the sclera tissue at the strain rates of (a) 5 and (b) $100 \mathrm{~mm} / \mathrm{min}$ from the examined strain energy density functions compared to the experimental as well as the finite element results. The number of subjects $(N=15)$ and the number of specimens $(N=30)$. A finite element model of the sclera tissue under the uniaxial tensile load was also established to compare the numerical and constitutive findings with that of the experimental ones (the inset of panel a). 

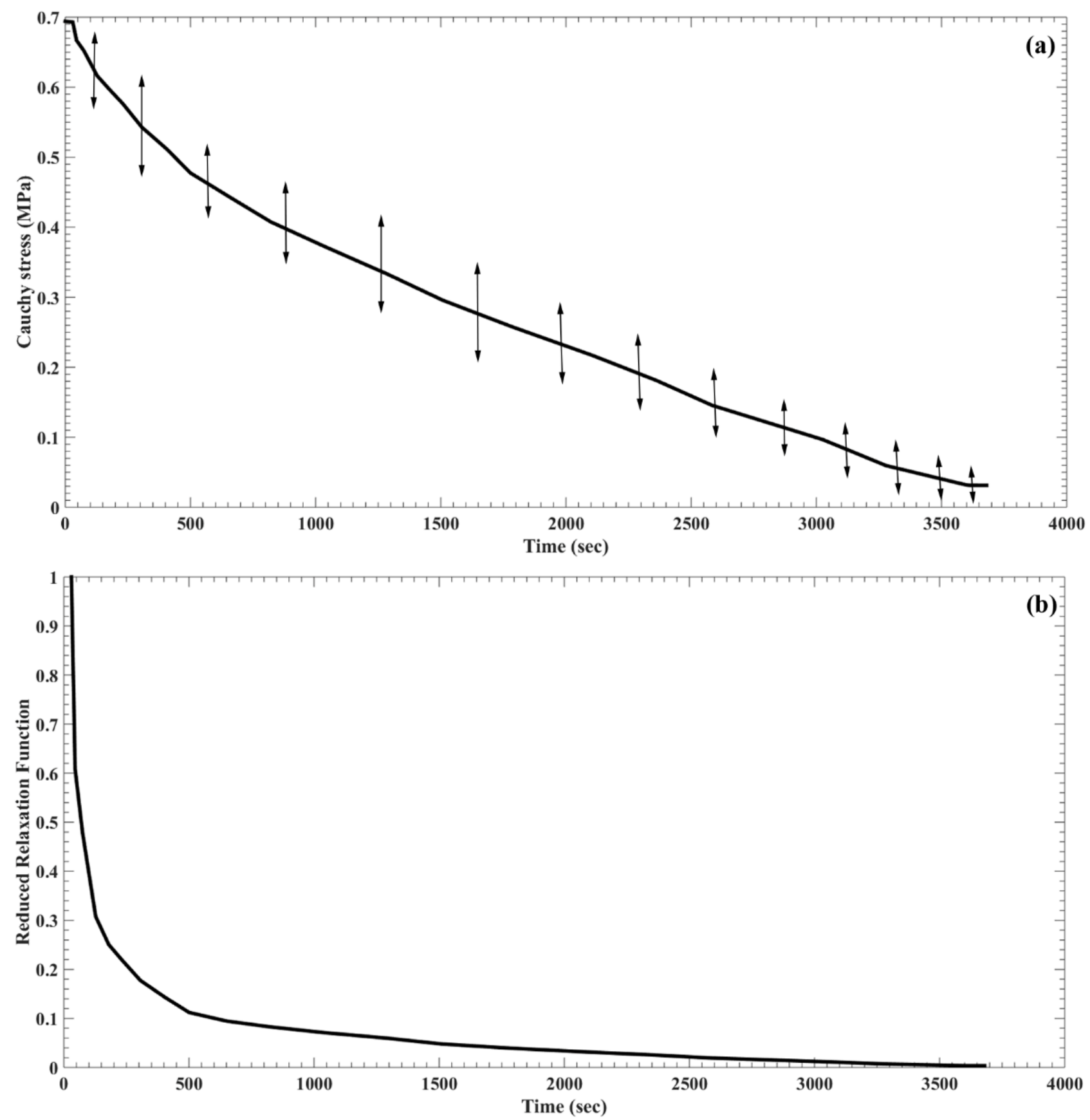

Fig. 20. The (a) stress-time response of the sclera tissue was measured through the stress-relaxation test. The (b) normalized reduced relaxation function versus time was also quantified and plotted. The number of subjects $(N=3)$ and the number of specimens $(N=6)$. 


\section{Chapter 3 \\ Computational Model of the Eye}


In this chapter, after knowing the mechanical properties of the sclera as the most important component of the eye, it is aimed at establishing an eye model on a basis of experimental $\mathrm{CT} / \mathrm{MRI} /$ literature data as well as experimental measurements on the sclera tissue.

\subsection{Eye model}

The morphological characteristics of the human eye (a normal/healthy male with the age of 59 year-old) were obtained from CT via 16 multislice CT/MRI System (Activion, Toshiba Medical Systems Corporation, Tokyo, Japan). Because of the incorporated $0.5 \mathrm{~mm}$ Quantum detector in this device with 350micron isotropic spatial resolution along with the best contrast resolution of $2 \mathrm{~mm}$ @ $0.3 \%$, the system ensures outstanding image quality in every examination at lowest X-ray dose. In addition, extremely low-dose scanning in combination with a fast scan protocol and Quantum De-noising software, enabled an entire head of $30-40 \mathrm{~cm}$ to be examined in just 10 seconds resulting in crystal clear, highest resolution images, further expanding diagnostic capabilities which is suitable for patients, specifically children. The donor declared his agreement to employ the CT images of his head for medical research purposes under the ethical rules of Tehran University of Medical Sciences based on the 2008 Declaration of Helsinki. $461 \mathrm{CT}$ images were attained with an especial focus on the eye. The images were then analyzed and transferred into the Digital Imaging and 
Communications in Medicine (DICOM) format. The CT images in this regard is presented in Fig. 21 with a focus on the eye.

The CT images then imported into the Mimics software. We had 461 image from one human head with a focus on the eye. Each image was analyzed in the software and using the segmentation in each stage the boundary of the globe was separated at each of 461 images. That is, the first image/slice was appeared on the screen of the software. According to the depth of the image from the head size, the elements of the eye including the lens, sclera/retina/vitreous body, cornea, and iris/aqueous body were divided. This process is shown in Fig. 22.

After segmentation on all the 461 CT images, the boundary of the eye for four components of the eye, including the cornea, aqueous body/iris, lens, sclera/retina/vitreous body. The images then using the Make Three-Dimensional (3D) model was combined and the 3D shape of the eye globe out of the Mimics software was constructed as illustrated in Fig. 23.

The model out of the Mimics software although seems to be similar to the real human eye in terms of the general structure, since its surfaces are not smooth enough, a FE software cannot provide a suitable meshing for that. To have smoother surfaces, from the CMF/Simulation of the Mimics software and choosing the smoothing model, the output model is prepared to be imported into the FE Abaqus software. It was almost impossible to recognize the components of the eye excluding the cornea, aqueous body, iris/aqueous body, lens, and sclera/retina/vitreous body, and incorporate them into the model. In the other 
words, the intra components of the eye, such as the vitreous body, ciliary body, retina, sclera, iris, and aqueous body by itself were totally not plausible to be distinguished in the DICOM images. This is why, other components were added into the model using the anatomical data of the eye reported in Vinegar [50] and Stitzel [12]. The anatomical model of the Stitzel is displayed in Fig. 2.

The CT/MRI and FE models of the eye components are displayed in Fig. 24. To make the exterior dimensions of the globe, two horizontal and vertical diameters along with the thickness of and length of the cornea separately were measured. In this figure, these two diameters for the CT/MRI data globe are presented and then incorporated into the FE model of the globe. However, there is a curvature in the anterior part of the cornea which its radius needs to be measured to be able to construct its shape. This shape in terms of the thickness in the apex of the cornea was determined via the CT/MRI data, while the radius of curvature in the side of the cornea was measured and constructed according to the model proposed by Stitzel. Thereafter, the next component in here is the cornea which its structure partly, excluding its curvature in the side area, was determined using the CT/MRI data. The thickness of $0.52 \mathrm{~mm}$ in the apex of the cornea is provided according to the thickness of the cornea reported by Vinegar/Stitzel data. The thickness and length were determined via the CT/MRI data. However, the curvature was defined according to the Stitzel data owing to the lack of accurate data in the CT/MRI data. The next components in the posterior of the cornea are the aqueous body and iris. Unfortunately, the CT/MRI data could not 
separate these two parameters and a unique component out of the imaging data was provided for us. This component was a combination of the iris and aqueous body. Since the Stitzel model also could not capture the dimension/structure of the iris, we used the literature which stated the thickness of $\sim 1.85 \mathrm{~mm}$ for the iris [51]. Since the thickness of this component was $2.98 \mathrm{~mm}$, therefore, $1.14 \mathrm{~mm}$ was dedicated to the aqueous body. That is, for the aqueous body since it is in direct contact to the cornea, the intra curvature of the cornea was used as a basis of the aqueous body's exterior curvature. Similarly, since the iris is in direct contact with the aqueous body from the anterior side, its curvature was made according to that. The next component in here is lens, which its structure was obtained from the CT/MRI data. Due to the suitable structure of the anterior part of the lens, its curvature was defined using the CT/MRI data, however, the posterior side of that was determined thru the symmetrical assumption of the lens.

The rest of the component of the eye in here is the combination of the vitreous body, retina, and sclera. The CT/MRI data in here only could measure the exterior part of the sclera which as we pointed out has the vertical diameter of $24 \mathrm{~mm}$. However, we needed to define the dimension of the sclera and retina. To do that, according to the Stitzel model, the thicknesses of the retina and sclera are almost $\sim 0.24-0.29$ [52] and 0.29-0.73 $\mathrm{mm}$ [53], respectively. However, in here due to the lack of accurate curvature it was considered to be fully symmetric and the model is idealized. The only component in here that we could not have its dimension was the ciliary body. However, we could have the dimension of the 
components which are in direct contact with that, including the lens, vitreous body, sclera, and iris.

The anatomical/image-based structure of our own proposed eye model on the basis of the experimental CT/MRI data and literature is displayed in Fig. 25. The proposed model in here as it was pointed out could not be established using only the CT/MRI data. Therefore, the dimension of some of the components of the eye, such as the vitreous body, aqueous body, ciliary body, retina, and iris, were partly extracted from the literature. It should be pointed out in the model proposed by Stitzel, the iris and retina were not taken into account, however, in the proposed model in here they are incorporated into the model.

Since the aqueous body in the eye is made of a liquid, our model must be a FSI model to be able to consider the interaction of the solid and fluid components of the eye. The FSI approach in brief is explained as following.

\subsection{Fluid-structure interaction coupling/arbitrary lagrangian-}

\section{eulerian}

The interfaces of Euler and Lagrange elements in the eye model were coupled using Arbitrary Lagrangian-Eulerian (ALE) method [54]. The ALE is a FE formulation in which the computational system is not a prior fixed in space (Eulerian) or attached to material (Lagrangian). ALE-based FE simulations can alleviate many of the drawbacks that the traditional Lagrangian-based and 
Eulerian-based FE simulations have. This coupling method could provide a suitable degree of freedom to establish an optimum mesh for the Lagrangian as well as Eulerian fields in comparison with substitute coupling approaches.

The ALE differential form of the conservation equations, for example, for the mass, momentum, and energy are sorted according to the well-known Eulerian forms:

Mass: $\quad \frac{d \rho}{d t}=\left.\frac{\partial \rho}{\partial t}\right|_{x}+v \cdot \nabla \rho=-\rho \nabla \cdot v$

Momentum:

$$
\rho \frac{d v}{d t}=\rho\left(\left.\frac{\partial v}{\partial t}\right|_{x}+(v \cdot \nabla) v\right)=\nabla \cdot \sigma+\rho b
$$

Energy:

$$
\rho \frac{d E^{*}}{d t}=\rho\left(\left.\frac{\partial E^{*}}{\partial t}\right|_{x}+v \cdot \nabla E^{*}\right)=\nabla \cdot(\sigma \cdot v)+v \cdot \rho b
$$

where $\rho$ is the mass density, $\mathrm{v}$ is the material velocity vector, $\sigma$ denotes the Cauchy stress tensor, $\mathrm{b}$ is the specific body force vector, and $\mathrm{E}^{*}$ is the specific total energy. If, for instance, the above energy equation is written in a form of

$\rho \frac{d e}{d t}=\rho\left(\left.\frac{\partial e}{\partial t}\right|_{x}+v \cdot \nabla e\right)=\sigma: \nabla^{s} v$

where in here e is the specific internal energy and $\nabla^{\mathrm{s}} \mathrm{v}$ denotes the stretching or strain tensor, the symmetric part of the velocity gradient $\nabla \mathrm{v}$; is $\nabla^{\mathrm{s}} \mathrm{v}=(1 / 2)\left(\nabla \mathrm{v}+\nabla^{\mathrm{T}} \mathrm{v}\right)$. In order to reach to the ALE form of the above equations, there is a need to replace the material velocity $\mathrm{v}$ with the convective velocity $c=$ $v-\hat{v}$. And the result is:

Mass:

$$
\left.\frac{\partial \rho}{\partial t}\right|_{x}+c \cdot \nabla \rho=-\rho \nabla \cdot v
$$


Momentum:

$$
\rho\left(\left.\frac{\partial v}{\partial t}\right|_{x}+(c . \nabla) v\right)=\nabla \cdot \sigma+\rho b
$$

Total energy:

$$
\rho\left(\left.\frac{\partial E}{\partial t}\right|_{x}+c \cdot \nabla E\right)=\nabla \cdot(\sigma \cdot v)+v \cdot \rho b
$$

Internal energy:

$$
\rho\left(\left.\frac{\partial e}{\partial t}\right|_{x}+c . \nabla e\right)=\sigma: \nabla^{S} v
$$

It is important to note that the right-hand side of equation is written in classical Eulerian form [55].

The contact algorithm exploited as the forces are computed to keep the slave nodes exactly on the master surface (zero penetration). Eroding contact types are recommended whenever solid elements involved in the contact definition are subject to erosion (element deletion) due to material failure criteria. These eroding contacts contain logic which allow the contact surface to be updated as exterior elements are deleted [56].

Regarding the Gruneisen equation of state for the Eulerian fluid, aqueous body, it defines pressure for a material in terms of relative volume and other coefficients as provided as following:

$p=\frac{\rho_{0} C^{2}\left[1+\left(1-\frac{\gamma_{0}}{2}\right) \mu-\frac{a}{2} \mu^{2}\right]}{\left[1-\left(S_{1}-1\right)-S_{2} \frac{\mu^{2}}{\mu+1}-S_{3} \frac{\mu^{3}}{(\mu+1)^{2}}\right]}$

where $C$ is the intercept of the Us-Up curve, $S_{1}, S_{2}$, and $S_{3}$ are the coefficients of the slope of the $\mathrm{U}_{\mathrm{s}}-\mathrm{U}_{\mathrm{p}}$ curve, $\gamma_{0}$ is the Gruneisen gamma, and a is the first order volume correction to $\gamma_{0}$. The compression, $\mu$, is defined in terms of the relative volume, $\mathrm{V}$, by $\mu=1 / \mathrm{V}-1$. Us is the speed of the shock wave through the material, and Up is the speed of the shocked material, both with respect to the rest frame 
of the un-shocked material. Thus, $\mathrm{C}$ is the speed of the sound through the material, or the velocity which a stress wave will propagate through the material at rest. The Jaumann stress rate is used with equation of state in LS-DYNA. In this approach, it computes the deviatoric strain rate tensor, and then propagates to evaluate the amount of stress in the components as a result of the rate of the applied load.

\subsection{Penetrating to the eye globe under blunt loading}

\subsubsection{Penetrating test procedure}

After getting the informed consent forms from each of the eye donors' family, the eyes of the human cadavers (twelve pairs) were enucleated between 5 and 10 hours after death and stored in a refrigerator at $4^{\circ} \mathrm{C}$. The number of eye globes were, therefore, twenty-four. The eyes were then transported to the mechanical measurements laboratory for the experimental procedures. The eye globes were taken out of the stored container and mounted on the testing machine. The experiments were then conducted within 3-5 hours of enucleation. In order to minimize the dislocation of the globe during the loading procedure, an appropriately prepared expanded polystyrene foam stand was employed to hold the eye globe. A measured screw, then fixed to the upper jaw of the machine and moved downward at five different speeds, including 5, 10, 20, 50, and 100 $\mathrm{mm} / \mathrm{min}$, to hit the eyeball. The process was carried out as a screw at five different speeds hit the cornea, which was located in the apex of the cornea, thereafter, the 
amount of the load as well as the displacement on that were recorded via a $50 \mathrm{kgf}$ load cell and a precise extensometer, respectively. Four eye globes were tested at the strain rate of $100 \mathrm{~mm} / \mathrm{min}$, while the rest of the samples were equally distributed to each strain rate. The tip of the screw was exactly located in a way to have an interaction with the apex of the cornea. This would help us to have better experimental measurements as well as lower amount of errors. The resulting displacement as well as the stress were finally calculated and reported. The fresh eye globe, experimental set-up, and failed eye globe are presented in Fig. 26. The elastic modulus is the ratio of alteration in the stress values in respect to the strain and, therefore, stands for the strength of a material. It defined as the initial linear/Hookean section of the stress-strain diagrams of the tissue responses to the applied load. The elastic modulus of the eyeball in here, as it is considered as a soft biological tissue, also calculated according to this law in the initial linear part of the stress-strain diagram up to the nonlinear region of that.

\subsubsection{Finite element model of the eye}

As it was pointed out, since the eye is composed of various components, different studies ignore some of the components of the eye in their proposed eye models. In order to figure out whether ignoring some of the components of the eye affect the results or not three different FE models of the human eye were established. The models were divided into four groups, including our model, $1^{\text {st }}$ 
model [57] (Fig. 27), the $2^{\text {nd }}$ model [58] (Fig. 28), the $3^{\text {rd }}$ model (based on the Stitzel Virginia Tech Eye Model (VTEM)) [12].

The models were established on a basis of the simplest ( $1^{\text {st }}$ model) up to the most advanced ones (our model and $3^{\text {rd }}$ model). The components of the eye models, including (a) our model, (b) the $1^{\text {st }}$ model, (c) the $2^{\text {nd }}$ model, and (d) the $3^{\text {rd }}$ model, are also introduced in Fig. 29.

The element type, material model, density, and material parameters of the human eye for our model, $1^{\text {st }}, 2^{\text {nd }}$, and $3^{\text {rd }}$ models are summarized in Tables 4, 5, 6, and 7, respectively. Six different mechanical properties were assigned to our model. The mechanical properties of the sclera were selected according to the mean mechanical properties under different strain rates. In addition, since we already performed a set of mechanical measurement on the human sclera tissue under different strain rates, i.e., 5, 10, 20, 50, and $100 \mathrm{~mm} / \mathrm{min}$, the calculated elastic modulus at each strain rate was assigned to the model with a related strain rate accordingly [59]. Assigning the related mechanical properties to the eye model at each screw/loading rate would help to have a better outlook on the deformation of the cornea. In our model, the cornea was posteriorly and anteriorly loaded with the IOP of $15 \mathrm{mmHg}$ and atmospheric pressure, respectively, ignoring the loading of the eyelids. The boundary of the IOP in that was set as an initial pressure which is acting on the components which are in direct contact with the aqueous body. In the $3^{\text {rd }}$ model, which was made according to the Stitzel model, the iris and aqueous body as well as the retina and sclera were combined 
(Fig. 29). Since in this section of our study it is objective to compute the amount of stresses and deformations as a result of blunt trauma impact, the components which are located in the anterior side of the side are deemed important.

Among the eye components, the ciliary body, lens, cornea, and sclera are ranked among the high strength eye components. However, the ciliary body was placed in the circumference and do not have a substantial role in load bearing of the eye from the anterior side. In the $1^{\text {st }}$ model, the eyeball was all made of a unique component with the elastic modulus as close as that of the cornea. Therefore, it is expected to see almost the same mechanical behavior in the initial zone of the numerical stress-strain diagrams. In the $2^{\text {nd }}$ model, the cornea includes the aqueous body and the iris, the ciliary body was replaced by the sclera, and the vitreous body was replaced with the elastic material instead of a viscoelastic material. With these considerations in mind, the $2^{\text {nd }}$ model in here was just made of the cornea, lens, vitreous body, and sclera. The reason why the $2^{\text {nd }}$ model only consisted of such components is that, these components of the eye due to their higher mechanical strength seem to have the most crucial role in bearing the blunt applied load. This is why this model was only benefitted from these components and the rest of the components owing to their insignificant mechanical properties were ignored. It is also helped us to shed light on the importance of having an almost a complete eye model, namely our model, comparing to the $1^{\text {st }}$ and $2^{\text {nd }}$ models, which are considered as the models with lower components or incomplete. The intra pressure as well as the vitreous part were suitably 
incorporated into the $1^{\text {st }}$ model. In our model, some simplifications were also unified into the current model of the human eye which may bound the model to provide the most accurate results we were expected to receive. The cornea was considered to be symmetric which enabled us to mirror the shape of a real healthy cornea $[29,60]$. The mechanical properties of the cornea were assumed to be nearly incompressible and linearly elastic [61]. In the $3^{\text {rd }}$ model, from the anterior side of the eye, the role of the iris, and from the posterior side, the roles of the retina and optic nerve, were all ignored. Such ignoring in the components of the eye model can confine the application of the model in blunt trauma impact as well as IOP alteration injuries simulations. Hence, it is believed that our own anatomical-based eye model due to considering the anterior and posterior components of the eye can have a broader application in such injuries simulations.

The model was smoothed in the SolidWorks, then meshed thru the FE-MAP, afterward incorporated into the Abaqus for assembling and remeshing. Finally, the model was inputted into the LS-DYNA software for the contact and material assignments as well as simulation.

\subsubsection{Numerical simulation}

The 8-node hexagonal elements were assigned to the eye components to be able to deliver us an in-plane mechanical properties for the shear modulus. The dynamic FE codes were solved using the nonlinear FE package and the obtained results in terms of the stresses and deformations were compared and reported. 
Regarding the boundary condition of the models, since the globe was hold by a structured expanded polystyrene (EPS) foam, in the numerical modeling as well the circumferential of the eye globes, which is the sclera, almost up to lower site of the cornea all three models were fixed in both planes upright to the screw's movement. Increasing the number of elements is supposed to improve the accuracy of the FE outcome. However, we need to make the elements as small as possible and get the best accuracy. The procedure had been carried out in a way that in order to have precise numerical results which have the lowest dependency to the number of elements and nodes, mesh convergence analyses have been carried out at three different numbers of elements excluding the coarse mesh at the strain rate of $100 \mathrm{~mm} / \mathrm{min}$. Thereafter, the outcomes in terms of the highest stress in the eye components, i.e., the cornea, lens, and sclera, were compared and the suitable number of elements was selected for the final modeling procedure. The number of elements for the eye model is provided in Table 8.

Thereafter, the suitable number of elements were selected in a way that the error of the FE solver with respect to its previous stage (mesh number) is lower than $2 \%$, the mesh density analysis stopped and the present ones considered as the most suitable number of elements. The amount of von Mises stress of these three eye components is reported in Fig. 30. It is obvious that, increasing the density of FE meshes although would lead to a better accuracy, it would also be accompanied with higher expense of longer analysis time due to higher number of nodes and elements. The number of elements and nodes for the eye model 
components finally have been selected as the $2^{\text {nd }}$ try (Table 8). Regarding the boundary condition, the nodes, where located in the bottom of the eye model, were fixed in all directions to be able to have a better establishment as close as possible to that of the real experimental procedure. In the experimental process the eye was hold by an expanded polystyrene foam container, however, they were not in full contact with each other excluding at the bottom side of the eye and, as a result, the eye was able to inflate in the circumference.

The simple elastic material model was used to address the mechanical behavior of the linear elastic eye components. This material type is defined based on the linear Hookean behavior in solid mechanics which the Young's modulus of the material connects the tensor of stress and strain together. The aqueous body was the only material model which its behavior was defined according to the MieGruneisen Equation of State (EOS) [62]. The contact algorithm utilized in this study was considered to be a coupling of the nodes to the surface while eroding or distortion is allowed between the contacted components. In the other words, this contact type lets the elements interfere with each other in the interaction site to be able to consider the role of element distortion in blunt trauma impact studies. As with previous studies [10], the vitreous body in the simple model was considered as a solid element with the pressure of $20 \mathrm{mmHg}(2.7 \mathrm{kPa})$ and the density of $1002 \mathrm{~kg} / \mathrm{m}^{3}$. So far two ways have been proposed to address the mechanical behavior of the vitreous body. They are including the simple pressure in an elastic solid ( $2^{\text {nd }}$ model) and the second approach is a viscoelastic model 
(our model and $3^{\text {rd }}$ model). In here, both approaches have been used and their values for the models are listed in Tables 4-7. Considering these three approaches also enabled us to have a comparative analysis on the role of eye components and their importance in the deformation of the cornea under the blunt loading. In our model and the $3^{\text {rd }}$ model, the IOP and atmospheric pressure were applied to the posterior and anterior sides of the cornea, respectively. In the $2^{\text {nd }}$ model, the intra pressure was acted on the sclera surface from the posterior and anterior sides as well as the lens from the anterior side (Fig. 29b). The nature of this pressure in here is load, hence, it could act freely on any part or surface. Thereafter, by applying the load on the cornea by the screw, the intra pressure could react and deform accordingly.

\subsubsection{Results}

\subsubsection{Experimental results}

The eye was subjected to a dynamic blunt screw at five different rates, including $5,10,20,50$, and $100 \mathrm{~mm} / \mathrm{min}$. The amounts of forces were found to be $31.37 \pm 1.10,50.21 \pm 1.39,38.94 \pm 1.47,70 \pm 2.03$, and $72 \pm 2.26 \mathrm{~N}(\mathrm{Mean} \pm \mathrm{SD})$ at the strain rates of $5,10,20,50$, and $100 \mathrm{~mm} / \mathrm{min}$, respectively. Similarly, the displacements of $10.32 \pm 0.55,9.81 \pm 0.39,9.65 \pm 0.62,10.34 \pm 0.73$, and $10.79 \pm 0.69$ $\mathrm{mm}$ at the strain rates of $5,10,20,50$, and $100 \mathrm{~mm} / \mathrm{min}$, respectively. The diameter of the screw was $5.44 \mathrm{~mm}$, therefore, the ranges of the force was found to be $31.37-72 \mathrm{~N}$ according to the rates of the applied load (5 to $100 \mathrm{~mm} / \mathrm{min}$ ). 
Recently, Lovald et al. [14] performed a FE analysis and experimental evaluation of penetrating injury through the cornea. They found that the eye globe fails under the force and displacement ranges of $30.50-58.20 \mathrm{~N}$ and $10.10-12 \mathrm{~mm}$, respectively, according to the size of the screw (1 and $2 \mathrm{~mm})$. Regardless of the differences among the experimental procedures of these two studies, our results show very good agreement comparing to their findings.

The mean stress-strain diagrams of the cornea under various strain rates were calculated and plotted in Fig. 31. The initial and failure condition of the eye under the applied load are also presented as the inset of Fig. 31. The initial refers to the globe before any load applied on that while the term failure expresses the final or last stage of load bearing which the eye globe yields under the applied load. The results enabled us to measure the macro static elastic modulus of the eye according to their stress-strain responses under various strain rates. The results in this regard are presented in Fig. 32. They showed that increasing the rate of the applied load can lead to a higher elastic modulus for the sclera tissue samples. That is, the elastic modulus of the sclera under the strain rate of $100 \mathrm{~mm} / \mathrm{min}$ was $49 \%$ higher than that of $5 \mathrm{~mm} / \mathrm{min}(p<0.05$, post hoc Scheffe method). The results, in addition, showed that by increasing the rate of the load up to 50 $\mathrm{mm} / \mathrm{min}$ the elastic modulus is also increasing accordingly. Yet, by increasing the rate of the load to $100 \mathrm{~mm} / \mathrm{min}$, no statistical difference was observed comparing to the elastic modulus under the strain rate of $50 \mathrm{~mm} / \mathrm{min}$. Since the current study was carried out at various rates of loadings, such as 5, 10, 20, 50, and 100 
$\mathrm{mm} / \mathrm{min}$, the results revealed the range of 1.33-2.13 MPa (Figs. 31 and 32) with the mean value of $1.66 \pm 0.35 \mathrm{MPa}(\mathrm{Mean} \pm \mathrm{SD})$. Although the difference among the elastic modulus at the strain rates of 5 to $20 \mathrm{~mm} / \mathrm{min}$ almost looked the same with $1.33-1.45 \mathrm{MPa}$, after the rate of $50 \mathrm{~mm} / \mathrm{min}$ a significant statistical increasing ( $p<0.05$, post hoc Scheffe method) was observed comparing to those of the low strain rates $(5,10$, and $20 \mathrm{~mm} / \mathrm{min})$. Regarding the maximum stress in the eyeball, an exactly the same trend was observed. In the other words, by increasing the rate of the applied load, a higher stress in the cornea was seen. This amount was $1.29 \pm 0.12 \mathrm{MPa}(\mathrm{Mean} \pm \mathrm{SD})$ at the strain rate of $5 \mathrm{~mm} / \mathrm{min}$ while $3.05 \pm 0.14 \mathrm{MPa}(\mathrm{Mean} \pm \mathrm{SD})$ at the strain rate of $100 \mathrm{~mm} / \mathrm{min}$ (Fig. 32) which is significantly ( $p<0.05$, post hoc Scheffe method) higher than that of the $5 \mathrm{~mm} / \mathrm{min}$. However, no statistical difference was observed between the strain rates of 50 and $100 \mathrm{~mm} / \mathrm{min}$ for the maximum stress. The mean value of these maximum stresses regardless of the strain rate was $2.31 \pm 0.75 \mathrm{MPa}(\mathrm{Mean} \pm \mathrm{SD})$. Thereafter, four totally different globe models were employed to compare the FE modeling results with that of the experimental ones. In here, the models called, our model, the $1^{\text {st }}, 2^{\text {nd }}$, and $3^{\text {rd }}$ (based on the Stitzel model) models, which were discussed earlier and illustrated in Fig. 29.

\subsubsection{Finite element results}

Using the proposed eye models, the experimental procedure was simulated using the nonlinear dynamic FE code. The results were then interpreted as the 
amount of stress at $1 \mathrm{~mm}$ deformation in the cornea in the element right under the screw and were, then, plotted and displayed in Fig. 33. The $1^{\text {st }}$ model in here was made of the unique component which its mechanical properties were extracted out of the experimental results of this study. The $2^{\text {nd }}$ model was made of the cornea, lens, sclera, and vitreous body while our model was consisted of the cornea, aqueous body, iris, ciliary body, lens, vitreous body, retina, and sclera. In order to be able to have a comparative analysis among the globe models in terms of their deformations under the applied blunt load, the deformed configurations of the eye models up to the deformation of $1 \mathrm{~mm}$ are presented in Fig. 29. These models well illustrated the deformation of the $1^{\text {st }}$ model which is consisted of a unique component. In addition, the $2^{\text {nd }}$ and our eye models, which in the $2^{\text {nd }}$ one the deformation was just involved with the cornea while in our model one the other anterior components of the eye were also involved. In the $3^{\text {rd }}$ model also almost all the components of the eye, excluding the iris and retina, were considered. However, since in here the blunt trauma impact was experimentally performed on the cornea and thereafter the same numerical simulations were conducted on the eye models, the anterior components of the eye play important roles. Since the assigned material properties into three models in here were all only could address the behavior of the eye components under the small deformation due to their linearly elastic nature, the proposed model could not well simulate the deformation of the eye under the large deformation. 
The resulted numerical stresses at $1 \mathrm{~mm}$ deformation in the apex of the cornea for the $1^{\text {st }}$ model were found to be $0.1 \mathrm{MPa}$ at all strain rates. However, the experimental data revealed the range of 0.03-0.06 $\mathrm{MPa}$ at all strain rates. The averaged value of the numerical stresses was $0.1 \mathrm{MPa}$ while for the experimental data it was $0.049 \pm 0.010 \mathrm{MPa}(\mathrm{Mean} \pm \mathrm{SD})$ which shows a significant difference. The $2^{\text {nd }}$ model showed the stress values of $0.27 \mathrm{MPa}$ at all the strain rates.

\subsubsection{Discussions}

In this study, the response of the eye globe to the applied blunt load was experimentally as well as numerically investigated. The anatomical based eye models were proposed for the numerical simulation, and then compared to the stress-displacement relationship among the experimental and numerical results. The results indicated that a simplified eye model is not suitable enough to address the mechanical response of the cornea under the blunt load and, consequently, all the components of the eye should be incorporated into the eye model even for a small deformation. To address our raised questions in the introduction section, in the latter sections the reasons of these differences are well discussed.

\subsubsection{Finite element results}

Regarding the FE models of the eye, the $1^{\text {st }}$ model was only made of a unique component which its mechanical properties were measured from the stress-strain response of the human eye globes under various strain rates. The mechanical 
properties of this model were considered to be simply linearly elastic. Therefore, it includes only the elastic modulus, maximum stress and strain, and Poisson's ratio. The elastic modulus of the eye globe is as close as that of the cornea and, as a result, it is expected to observe small difference among the $1^{\text {st }}$ and $2^{\text {nd }}$ eye models. Because $1 \mathrm{~mm}$ deformation in the cornea in the $1^{\text {st }}$ and $2^{\text {nd }}$ eye models almost exclusively concentrated in the initial or the most anterior component of the eye, which is the cornea. According to Fig. 33 , the $1^{\text {st }}$ model, which is the simplest model of the eye, showed a disagreement between the resulted experimental data. The resulted average stress at $1 \mathrm{~mm}$ deformation in the apex of the cornea was reported to be $0.10 \mathrm{MPa}$ while the experimental one was $0.049 \pm 0.010 \mathrm{MPa}(\mathrm{Mean} \pm \mathrm{SD})$. Regardless of a lower or higher deformation, the mean stress in the $1^{\text {st }}$ model was $104 \%$ higher than that of the experimental data (Fig. 33). The mechanical properties of the eyeball in here were all experimentally measured and assigned to the FE model. However, it should also be noted that why there is a difference among the experimental data and that of the $1^{\text {st }}$ model while the same mechanical properties have incorporated into the model. In the $1^{\text {st }}$ model only a simple elastic component was represented while the eye is a very complex organ with different intricate components that even in a small deformation of the eyeball contribute in load bearing. In addition, the proposed model in here was compared to the $3^{\text {rd }}$ model as presented in Fig. 29 with the material properties of Table 7 . The results revealed that the amount of stresses at the determined displacements up to $1 \mathrm{~mm}$ are lower than that of the 
experimental data. Although the stress-strain results showed the same trend as the experimental data, due to not considering the iris in their model and combining the iris and the aqueous body together it could not bear the same load as a combination of solid (iris) and fluid (aqueous body) bear. This is why such disagreement in their stress-strain results compared to the experimental data was found.

The $2^{\text {nd }}$ and our models have so many similarities while the number of components in their structures is different. Their cornea and lens both have the same mechanical properties while the mechanical properties of the sclera, vitreous body, and sclera are different. The sclera in the $1^{\text {st }}$ model was obtained from the literature while in our model it was measured experimentally by the authors. At first, in order to figure out why there is a difference among the models' response to the applied load and their differences compared to the experimental data, the stresses and deformations of three components of the models were compared under the strain rate of $100 \mathrm{~mm} / \mathrm{min}$ as a representative (Fig. 34 and Table 9).

In the $2^{\text {nd }}$ model, the lens from the top side is in direct contact with the cornea while in our model it is in contact with the iris. The results in here showed that for these two models the displacement and stress in the lens for the $3^{\text {rd }}$ model was higher compared to the $2^{\text {nd }}$ one (Table 9). The reason as discussed is related to the upper component in the $3^{\text {rd }}$ model which is the liquid (aqueous body) and then a solid (iris with the elastic modulus of $4 \mathrm{kPa}$ ). Due to the hydrostatic pressure in 
the aqueous body, the cornea in our model contacted with a liquid which is softer to deform compared to the iris in the $2^{\text {nd }}$ model. As a result of a higher deformation in the aqueous body of the $3^{\text {rd }}$ model as well as according to the law of solid mechanics, a higher deformation in the lens of our model in comparison to the lens of the $2^{\text {nd }}$ model is expected. These structural differences may also affect the ciliary body. The ciliary body in the $2^{\text {nd }}$ model was made of the sclera's material while in our model it is made by a real ciliary body material. The ciliary body in our model showed the deformation $(0.045 \mathrm{~mm})$ which is higher than that of the $2^{\text {nd }}$ model $(0.011 \mathrm{~mm})$. Although the mechanical property of our model is stiffer than that of the $2^{\text {nd }}$ model, the displacement is larger in our model (Table 9). That is, the ciliary body in the $2^{\text {nd }}$ model both because of its location and mechanical properties could relatively bear a smaller deformation compared to our model's ciliary body.

The displacement and stress of the vitreous body as the most posterior component of the eyeball for the $2^{\text {nd }}$ and our models were also investigated and revealed a higher deformation in the vitreous body for our model $(0.08 \mathrm{~mm})$ comparing to the $2^{\text {nd }}$ one $(0.0018 \mathrm{~mm})$. The mechanical properties of these two components were different in these two models, as the $2^{\text {nd }}$ model benefitted from the elastic while our model from the viscoelastic materials. As a result of this difference, the resulted mechanical deformations as well as the stresses are different. As it is known, the nature of the vitreous body is viscoelastic, however, in the $2^{\text {nd }}$ model it was considered to behave as an elastic material [10]. As a result 
of an elastic vitreous body incorporation into the model, it could not well react to the applied blunt load in the cornea and, subsequently, it led to the disagreement which is visible in the $2^{\text {nd }}$ model of Fig. 33. In addition to that, the expansion or any type of deformation in the vitreous body also contributes to the deformation of the sclera. The mechanical properties of the sclera in the $2^{\text {nd }}$ and our models are different (Tables 6 and 4). In the $2^{\text {nd }}$ model the sclera's properties taken from the literature while in the $3^{\text {rd }}$ model it was extracted from the chapter 3 . Furthermore, the structure of the sclera in these two eye models are different as in the $2^{\text {nd }}$ model it is also cover the ciliary body whereas in the our model it is a separate component. Sclera is the most outer component of the eye and its role is a holder of intra components of the eye. Therefore, it is of vital importance to figure out the amount of variation in its diameter after $1 \mathrm{~mm}$ deformation in the cornea. The initial diameter of the eye globe as displayed in Fig. 34 was set to $24.0076 \mathrm{~mm}$. After applying the load in the cornea up to $1 \mathrm{~mm}$ deformation in the $2^{\text {nd }}$ and our models, it reached to the values of 24.0079 and $24.0250 \mathrm{~mm}$, respectively. In fact, the displacement at vitreous body in $2^{\text {nd }}$ and our models are $0.0018 \mathrm{~mm}$ and $0.08 \mathrm{~mm}$ (Table 9), respectively, resulting in that the diameter might be larger in our model. The diameter of the globe in here was not considerably $(0.07 \%)$ affected by the mechanical properties of the sclera which is the holder or container of the eye components inside the globe. Since the mechanical properties of the eye globe in the $2^{\text {nd }}$ model is 5 times higher than that of our model, such small difference was observed. However, it should be noted 
that our results only reported under the small deformation of the cornea $(1 \mathrm{~mm})$, and there is no question that a higher deformation in the cornea would substantially affect the diameter change in the eye globe. The mechanical properties of the sclera in our model was benefitted from the experimental ones of the human eye at various loading rates according to the rate of the blunt loading in contrast to the $2^{\text {nd }}$ model. Furthermore, it should note that considering the vitreous body as an elastic material would trigger unsuitable numerical findings for the $2^{\text {nd }}$ model according to our outcomes about the diameter of the sclera in this model. Therefore, there is a need to compensate this disagreement by incorporating the suitable material properties of the vitreous body into the eye models.

Regarding other components, such as the cornea, aqueous body, and iris, they reacted to the applied load and, consequently, due to the fluidic nature of the aqueous body it could move freely in any direction. In here, according to Fig. $29 \mathrm{c}$, it is observed that after the interaction of the screw with the cornea and applying the load, the cornea was well deformed and also part of the aqueous body was also involved in this deformation. Because it is in direct contact with the cornea from its posterior side, the aqueous body was also distorted in addition to the cornea (Fig. 29c). This may also reflect the reason of disagreement at the initial of the stress-displacement diagrams between the $1^{\text {st }}$ and $2^{\text {nd }}$ models and that of the $3^{\text {rd }}$ model. That is, in the $3^{\text {rd }}$ model, the screw should penetrate into a cornea which is being supported by a liquid type material from its posterior side, and that 
is why its result is different comparing to the other two models. The cornea in our model was posteriorly supported by the aqueous body while in the $2^{\text {nd }}$ model it was supported by a solid component (Figs. 29b and c). The same definition can be provided for the $3^{\text {rd }}$ model, as in that due to not considering the iris which is located in the posterior side of the aqueous body, the amount of stress at the same displacement was a bit lower than that of our model. In this case, which is the example of blunt trauma impact, the important role of the iris in load bearing was obviously observed.

The results of the current study revealed that it is crucial to consider all the component of the eye into a FE model since the results were affected by the number of components. As it was pointed out, so far almost four different models, including Uchio et al. [10, 11], Stitzel et al. [12], Rossi et al. [13], and Lovald et al. [14], have been proposed and employed in the FE simulations of the eye. Although each of which has its own advantages and disadvantages, one thing is common among them which is the lack of considering all the components of the eye into their model. The question that we raised was that do their numerical results affect by not considering some of the eye components or not?. To answer this question three eye models as the simplest ( $1^{\text {st }}$ model) up to the most complete one (our and $3^{\text {rd }}$ models) were proposed. In the $1^{\text {st }}$ and $2^{\text {nd }}$ eye models some of the components of the eye were ignored whereas our and $3^{\text {rd }}$ models were benefitted from almost all the components of the eye. The stress in the cornea after $1 \mathrm{~mm}$ deformation were then compared and, a disagreement was observed for the $1^{\text {st }}$ 
and $2^{\text {nd }}$ eye models while our model presented the most suitable agreement. According to the results it is obvious that each component of the eye has its own role in the contribution of load bearing even under a very small deformation (1 $\mathrm{mm})$. In the model proposed by the Uchio, for example, the aqueous and vitreous bodies were considered to be elastic materials while our results in the $2^{\text {nd }}$ and our models indicated that the aqueous and vitreous bodies have substantial roles in the initial and later deformation of the eye globe. Stitzel did not consider the iris and retina in the model due to their small elastic modulus, while our results in the $2^{\text {nd }}$ model showed that not considering these components of the eye affect the numerical findings. In here also we could show that the $3^{\text {rd }}$ model due to not considering the role of iris failed to address the deformation of the cornea under even small deformation. Although the mechanical properties of the aqueous and vitreous bodies in the Rossi's model were considered to be liquid with EOS function as well as Eulerian element type, the roles of iris and ciliary body were ignored in their model. For instance, our results in terms of the displacement and stress of the ciliary body in both the $2^{\text {nd }}$ and our eye models showed more than 3 times difference. It implies that even different mechanical properties for this component of the eye can substantially affect the results and, this is why, it is inferred that the role of ciliary body in load bearing of the eye under even a small mechanical deformation is deemed important. Regarding the latter model proposed by Lovald, it is shown that the roles of the aqueous body as well as vitreous body were ignored; and the sclera and retina were considered to be made 
of a unique component. The comparative results among the $2^{\text {nd }}$ and our eye models in the current research revealed that the aqueous and vitreous bodies have pivotal roles in the initial and latter deformation of the cornea under the penetrating load. For example, our results exhibited that the displacement of the vitreous body in the $2^{\text {nd }}$ model is 4 times higher than that of our model. This is why their roles have to be considered into an eye model.

\subsubsection{Experimental results}

Although there are some scattering effects due to the nature of the eye globe, the results illustrated that a higher strain rate can almost lead to a higher stress. For example, the stress of $1.29 \mathrm{MPa}$ was observed at the strain rate of $5 \mathrm{~mm} / \mathrm{min}$ while the stress of $3.05 \mathrm{MPa}$ was seen at the strain rate of $100 \mathrm{~mm} / \mathrm{min}$. This mechanical behavior seems to be common among the soft biological tissues since, for instance, the coronary arteries also showed a higher stress and lower strain failures by increasing the rate of the applied load [63]. Moreover, all the curves in here showed a parabolic shape with an increasing rate. This phenomenon is also common among the soft biological tissues and defined as softening, which is by increasing the strain, the stress is increasing accordingly. Elastic modulus of the eye globe may have so many applications in the modeling studies since it can be used as a simple material model that can be assigned to the human eye for different types of numerical/mechanical simulations [64, 65]. In addition, existing models lack of accurate stress-strain properties (mechanical properties) for ocular 
globe under various loading rates. The current study carried out at various rates of loadings, such as $5,10,20,50$, and $100 \mathrm{~mm} / \mathrm{min}$, the results revealed the range of 1.33-2.13 MPa (Figs. 31 and 32) with the mean value of $1.66 \pm 0.35 \mathrm{MPa}$ (Mean $\pm \mathrm{SD})$. Recent studies reported the average range of $1.76-11.17 \mathrm{MPa}$ as the elastic modulus of the human globe under static loading [66]. The results of this section suggested that if the aim of a study is to have a macroscopic deformation of the cornea regardless of the exact stress in each component of the eye, the $1^{\text {st }}$ model due to its simplicity can be preferable. However, if the objective is to find the amount of stress in all the components of the eye in detail, then, our model is deemed necessary. These results may have associations not only for knowing the amount of von Mises stress brings about globe rupture in the eye but also for providing a wide range of information regarding that under various loading rates.

So far we could figure out which eye model is suitable for injury simulation under the blunt impact load and can follow the pattern of experimental results. Now it is aimed at understanding the result of IOP alteration on the amount of stress and deformation in other components of the eye. In addition, since the optic nerve/sclera made of a single material properties, the role of the sclera's mechanical properties in an eye model when it subjects to an IOP over than the normal range was investigated. This will have implication not only for knowing the importance of the sclera's mechanical properties in the eye model but also for understanding the $\mathrm{ONH}$ biomechanics as a result of IOP increasing. 
Table 4. The element type, material model, density, and material parameters of our eye model.

\begin{tabular}{|c|c|c|c|c|}
\hline Model component & $\begin{array}{c}\text { Element } \\
\text { type }\end{array}$ & $\begin{array}{c}\text { Material } \\
\text { model }\end{array}$ & $\begin{array}{l}\text { Density } \\
\left(\mathrm{kg} / \mathrm{m}^{3}\right)\end{array}$ & $\begin{array}{c}\text { Material parameters } \\
\text { (Reference) }\end{array}$ \\
\hline Cornea & Lagrangian & Elastic & 1143 & $\begin{array}{c}\mathrm{E}=1.50 \mathrm{MPa}, \mathrm{S}_{\mathrm{y}}=9 \mathrm{MPa}, \\
v=0.47[13]\end{array}$ \\
\hline Aqueous body & ALE & Shock EOS & 1000 & $\begin{array}{c}\mu^{*}(\text { viscosity })=7.50 \times 10^{-4} \\
\text { Pa.s [67], } C=1530 \mathrm{~m} / \mathrm{s} \\
\mathrm{S}_{1}=2.10, \mathrm{~S}_{2}=-0.1744 \\
\mathrm{~S}_{3}=0.010085, \gamma_{0}=1.20 \\
{[12]}\end{array}$ \\
\hline Iris & Lagrangian & Elastic & 1000 & $\mathrm{E}=4 \mathrm{kPa}, v=0.47[68]$ \\
\hline Ciliary body & Lagrangian & Elastic & 1600 & $\mathrm{E}=11 \mathrm{MPa}, v=0.47[69]$ \\
\hline Lens & Lagrangian & Elastic & 1078 & $\begin{array}{c}\mathrm{E}=6.88 \mathrm{MPa}, v=0.47 \\
{[70]}\end{array}$ \\
\hline Vitreous body & Lagrangian & Viscoelastic & 950 & $\begin{array}{c}\mathrm{G}_{0}=10 \mathrm{~Pa}, \mathrm{G}_{\infty}=2 \mathrm{~Pa}, \\
\beta=0.01 \mathrm{~s}^{-1}, \mathrm{~K}=2000 \mathrm{MPa} \\
{[13]}\end{array}$ \\
\hline Retina & Lagrangian & Elastic & 1110 & $\mathrm{E}=20 \mathrm{kPa}, \mathrm{v}=0.47[71]$ \\
\hline Sclera $(5 \mathrm{~mm} / \mathrm{min})$ & Lagrangian & Elastic & 1243 & $\begin{array}{c}\mathrm{E}=1.10 \mathrm{MPa}, v=0.47 \\
{[59]}\end{array}$ \\
\hline Sclera $(10 \mathrm{~mm} / \mathrm{min})$ & Lagrangian & Elastic & 1243 & $\begin{array}{c}\mathrm{E}=1.97 \mathrm{MPa}, v=0.47 \\
{[59]}\end{array}$ \\
\hline Sclera $(20 \mathrm{~mm} / \mathrm{min})$ & Lagrangian & Elastic & 1243 & $\begin{array}{c}\mathrm{E}=1.81 \mathrm{MPa}, v=0.47 \\
{[59]}\end{array}$ \\
\hline Sclera $(50 \mathrm{~mm} / \mathrm{min})$ & Lagrangian & Elastic & 1243 & $\begin{array}{c}\mathrm{E}=1.77 \mathrm{MPa}, v=0.47 \\
{[59]}\end{array}$ \\
\hline Sclera $(100 \mathrm{~mm} / \mathrm{min})$ & Lagrangian & Elastic & 1243 & $\begin{array}{c}\mathrm{E}=1.61 \mathrm{MPa}, v=0.47 \\
{[59]}\end{array}$ \\
\hline Rigid object (screw) & Lagrangian & Elastic & 7800 & $\mathrm{E}=210 \mathrm{GPa}, v=0.30[72]$ \\
\hline Optic nerve & Lagrangian & Elastic & 1243 & $\mathrm{E}=1.65 \mathrm{MPa}, v=0.47$ \\
\hline Muscle & Lagrangian & Elastic & 1060 & $\mathrm{E}=40 \mathrm{kPa}, v=0.47[57]$ \\
\hline Intraconal fat & Lagrangian & Elastic & 970 & $\begin{array}{c}\mathrm{E}=0.30 \mathrm{kPa}, v=0.48[73, \\
74]\end{array}$ \\
\hline Extraconal fat & Lagrangian & Viscoelastic & 970 & $\begin{array}{c}\mathrm{G}_{0}=0.90 \mathrm{kPa}, \mathrm{G}_{\infty}=0.50 \\
\mathrm{kPa}, \beta=50 \mathrm{~s}^{-1}, \mathrm{~K}=2000 \\
\operatorname{MPa}[73,74]\end{array}$ \\
\hline Tennis ball & Lagrangian & Hyperelastic & 1060 & $\begin{array}{c}\mu_{1}=-15.77 \mathrm{MPa} \\
\mu_{2}=7.84, \mu_{3}=8.04, \alpha_{1}=- \\
3.47, \alpha_{2}=-2.33, \alpha_{3}=-4.65 \\
{[75]}\end{array}$ \\
\hline
\end{tabular}

Note: $E$, elastic modulus; $\mathrm{S}_{\mathrm{y}}$, Yield stress; $v$, Poisson's ratio; $A L E$, Arbitrary Lagrangian Eulerian; $\mu$, viscosity; $C$, speed of sound through the material; $s_{1}$, the coefficient related to the speed of the shocked material; $\gamma_{0}$, the Grüneisen gamma; e, the internal energy; $G_{0}$, initial shear modulus; $G_{\infty}$, long time shear modulus; $\beta$, viscoelastic decay constant; $K$, bulk modulus. 
Table 5. The element type, material model, density, and material parameters of the $1^{\text {st }}$ eye model.

\begin{tabular}{lcccc}
\hline Model component & $\begin{array}{c}\text { Element } \\
\text { type }\end{array}$ & $\begin{array}{c}\text { Material } \\
\text { model }\end{array}$ & $\begin{array}{c}\text { Density } \\
\left(\mathrm{kg} / \mathrm{m}^{3}\right)\end{array}$ & $\begin{array}{c}\text { Material parameters } \\
\text { (Reference) }\end{array}$ \\
\hline Eye globe & Lagrangian & Elastic & 1000 & $\begin{array}{c}\mathrm{E}=2.20 \mathrm{MPa}, v=0.47 \\
\mathrm{E}=210 \mathrm{GPa}, v=0.30\end{array}$ \\
Rigid object & Lagrangian & Elastic & 7800 & {$[72]$} \\
\hline
\end{tabular}

Note: $E$, elastic modulus; $v$, Poisson's ratio. Elastic modulus: it is the mean elastic modulus of all samples at different strain rates; The Poisson's ratio is defined according to the previous results reported for the eye globe since our test was just uniaxial we could not measure this value out of our own experiment. 
Table 6. The element type, material model, density, and material parameters of the $2^{\text {nd }}$ eye model.

\begin{tabular}{|c|c|c|c|c|}
\hline Model component & $\begin{array}{l}\text { Element } \\
\text { type }\end{array}$ & $\begin{array}{c}\text { Material } \\
\text { model }\end{array}$ & $\begin{array}{l}\text { Density } \\
\left(\mathrm{kg} / \mathrm{m}^{3}\right)\end{array}$ & $\begin{array}{c}\text { Material parameters } \\
\text { (Reference) }\end{array}$ \\
\hline Cornea & Lagrangian & Elastic & 1143 & $\begin{array}{c}\mathrm{E}=1.50 \mathrm{MPa}, \mathrm{S}_{\mathrm{y}}=9 \\
\mathrm{MPa}, v=0.47[13]\end{array}$ \\
\hline Lens & Lagrangian & Elastic & 1078 & $\mathrm{E}=6.88 \mathrm{MPa}, v=0.47$ \\
\hline Vitreous body & Lagrangian & Elastic & 1002 & $\begin{array}{c}\tau_{\text {shear modulus }}=4.55 \mathrm{~Pa}, \\
\mathrm{E}=13.61 \mathrm{~Pa}, \mathrm{v}=0.495 \\
{[21]}\end{array}$ \\
\hline Sclera & Lagrangian & Elastic & 1243 & $\begin{array}{c}\mathrm{E}=5.50 \mathrm{MPa}, v=0.47 \\
{[10]}\end{array}$ \\
\hline Rigid object & Lagrangian & Elastic & 7800 & $\begin{array}{c}\mathrm{E}=210 \mathrm{GPa}, v=0.30 \\
{[72]}\end{array}$ \\
\hline
\end{tabular}

Note: $E$, elastic modulus; $\mathrm{S}_{\mathrm{y}}$, Yield stress; $v$, Poisson's ratio. 
Table 7. The element type, material model, density, and material parameters of the $3^{\text {rd }}$ eye model [12].

\begin{tabular}{|c|c|c|c|c|}
\hline Model component & $\begin{array}{c}\text { Element } \\
\text { type }\end{array}$ & $\begin{array}{l}\text { Material } \\
\text { model }\end{array}$ & $\begin{array}{l}\text { Density } \\
\left(\mathrm{kg} / \mathrm{m}^{3}\right)\end{array}$ & $\begin{array}{l}\text { Material parameters } \\
\quad \text { (Reference) }\end{array}$ \\
\hline Cornea & Lagrangian & Elastic & 1000 & $\begin{array}{c}\mathrm{E}=1.50 \mathrm{MPa}, \mathrm{S}_{\mathrm{y}}=9 \\
\mathrm{MPa}, \nu=0.47\end{array}$ \\
\hline Aqueous body & ALE & Shock EOS & 1000 & $\begin{array}{c}\mu^{*}(\text { viscosity })=7.50 \times 10^{-} \\
{ }^{4} \text { Pa.s, } C=1530 \mathrm{~m} / \mathrm{s} \\
\mathrm{s}_{1}=2.10, \mathrm{~S}_{2}=-0.1744 \\
\mathrm{~S}_{3}=0.010085, \gamma_{0}=1.20\end{array}$ \\
\hline Ciliary body & Lagrangian & Elastic & 1600 & $\mathrm{E}=11 \mathrm{MPa}, v=0.47$ \\
\hline Lens & Lagrangian & Elastic & 1078 & $\mathrm{E}=6.88 \mathrm{MPa}, v=0.47$ \\
\hline Vitreous body & Lagrangian & Viscoelastic & 950 & $\begin{array}{c}\mathrm{G}_{0}=10 \mathrm{~Pa}, \mathrm{G}_{\infty}=2 \mathrm{~Pa} \\
\beta=0.01 \mathrm{~s}^{-1}, \mathrm{~K}=2000 \\
\mathrm{MPa}\end{array}$ \\
\hline Zonules & Lagrangian & Elastic & 1000 & $\mathrm{E}=357.78 \mathrm{MPa}, v=0.47$ \\
\hline Sclera & Lagrangian & Elastic & 1400 & $\mathrm{E}=5.50 \mathrm{MPa}, v=0.47$ \\
\hline Rigid object & Lagrangian & Elastic & 7800 & $\begin{array}{c}\mathrm{E}=210 \mathrm{GPa}, \nu=0.30 \\
{[72]}\end{array}$ \\
\hline
\end{tabular}

Note: $E$, elastic modulus; $v$, Poisson's ratio. 
Table 8. The number of elements of the $3^{\text {rd }}$ eye model components at three different mesh densities.

\begin{tabular}{lccc}
\hline Model component & $\begin{array}{c}1^{\text {st }} \text { try } \\
\text { Number of } \\
\text { elements }\end{array}$ & $\begin{array}{c}2^{\text {nd }} \text { try } \\
\text { Number of } \\
\text { elements }\end{array}$ & $\begin{array}{c}3^{\text {rd }} \text { try } \\
\text { Number of } \\
\text { elements }\end{array}$ \\
\hline Cornea & 2135 & 3664 & 4368 \\
Aqueous body & 1956 & 3120 & 3978 \\
Iris & 4112 & 6240 & 7110 \\
Ciliary body & 3294 & 5120 & 6098 \\
Lens & 6234 & 8512 & 9112 \\
Vitreous body & 28512 & 37776 & 42598 \\
Retina & 4529 & 6832 & 8014 \\
Sclera & 4899 & 6176 & 7110 \\
Optic nerve & 2436 & 3840 & 4589 \\
Muscle & 1710 & 2904 & 3614 \\
Intraconal fat & 12415 & 16896 & 18115 \\
Extraconal fat & 15896 & 19472 & 23232 \\
Rigid object & 1525 & 2598 & 3255 \\
\hline
\end{tabular}


Table 9. The amounts of displacement and stress in some of the components of the 2 ns and $3^{\text {rd }}$ eye models.

\begin{tabular}{llcc}
\hline Component & & Displacement $(\mathrm{mm})$ & Stress (MPa) \\
\hline \multirow{2}{*}{ Lens } & $2^{\text {nd }}$ model & 0.0235 & 0.048 \\
& $3^{\text {rd }}$ model & 0.080 & 0.06 \\
\hline \multirow{2}{*}{ Vitreous body } & $2^{\text {nd }}$ model & 0.0018 & $4.1 \mathrm{e}-5$ \\
& $3^{\text {rd }}$ model & 0.08 & $9 \mathrm{e}-7$ \\
\hline \multirow{2}{*}{ Ciliary body } & $2^{\text {nd }}$ model & 0.011 & 0.013 \\
& $3^{\text {rd }}$ model & 0.045 & 0.045 \\
\hline
\end{tabular}

The location of the elements in the lens, vitreous body, and ciliary body, that their displacement and stress were measured, is displayed in Fig. 34. 


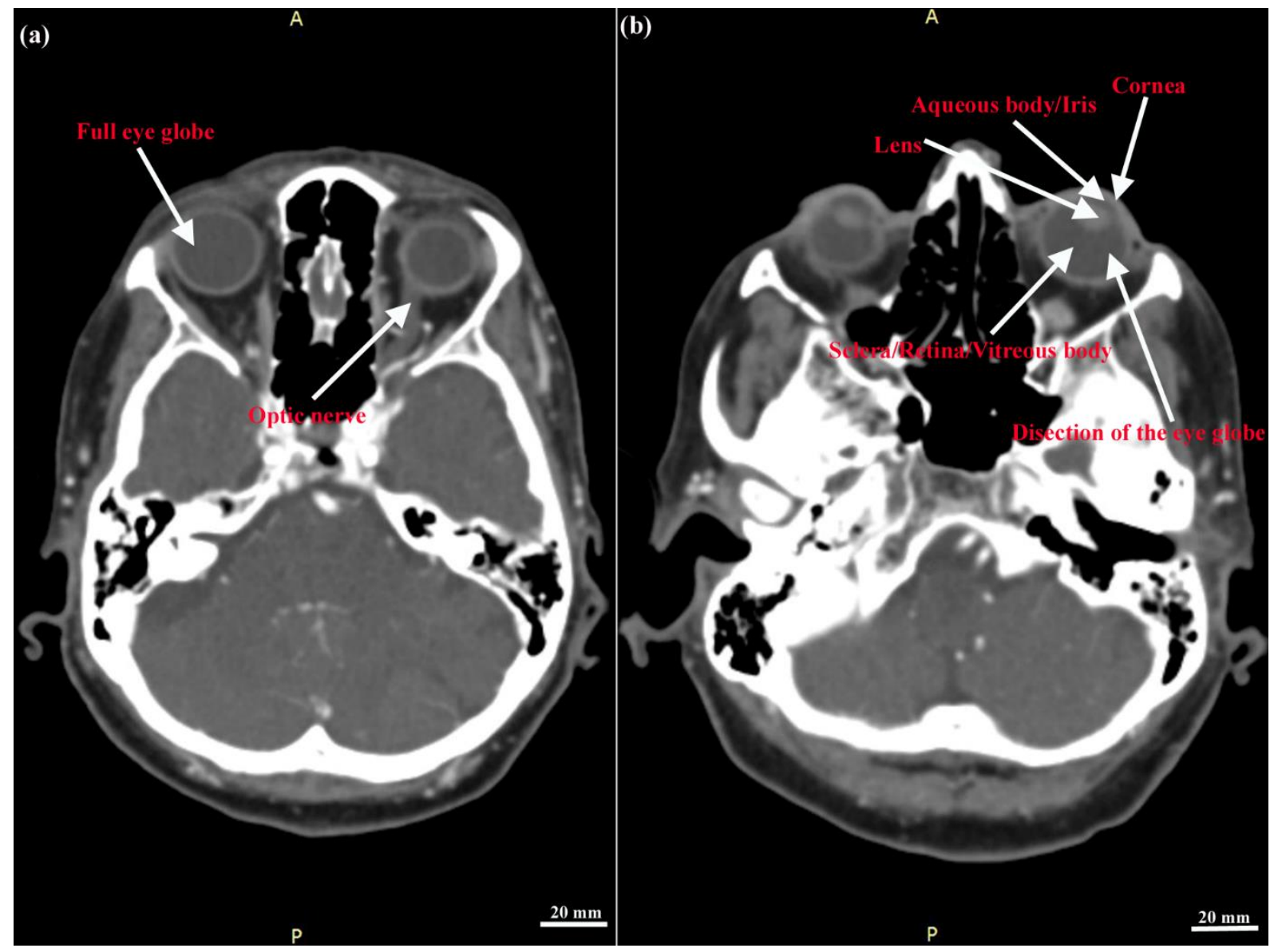

Fig. 21. The CT/MRI data of the human head. (a) the full eye globe as well as the

(b) dissected eye globe. In addition, some components of the eye, such as the optic nerve, cornea, aqueous body/iris, sclera/retina/vitreous body, and lens are displayed in this images. 


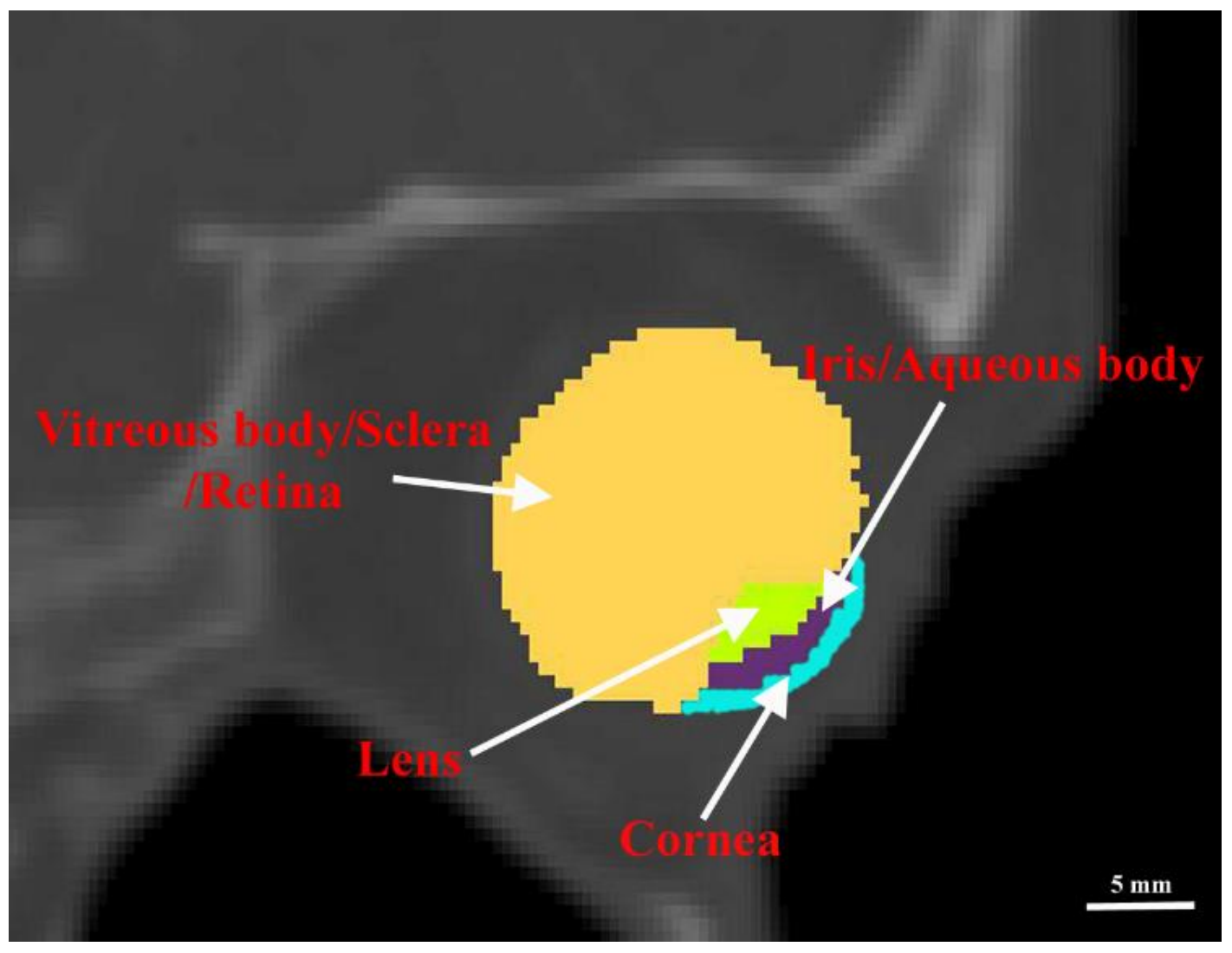

Fig. 22. The CT/MRI data of the human eye. The eye components in this regard then separated at each of 461 images. This image shows one slide out of 461 ones and the same process was done on each image via segmentation. 


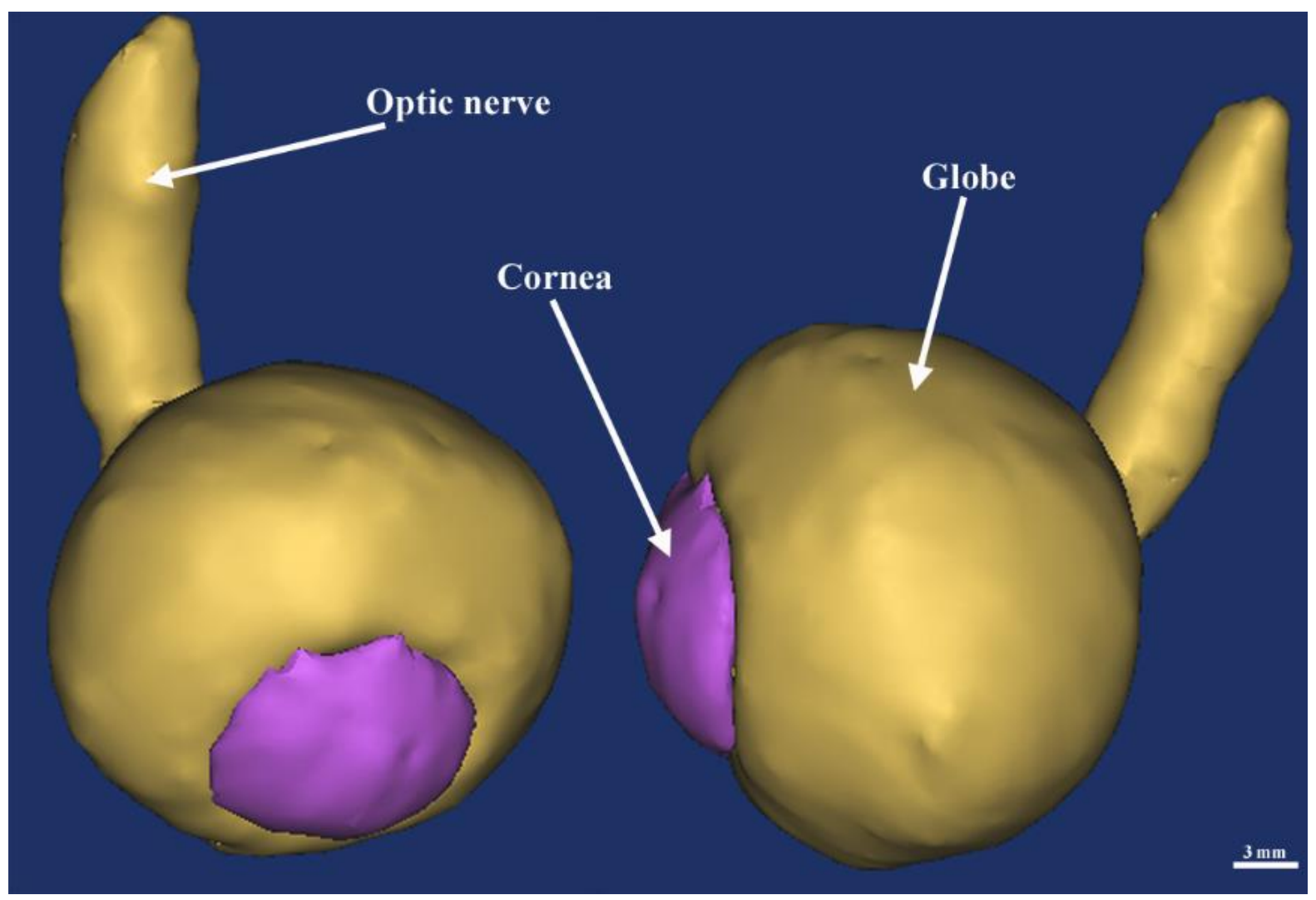

Fig. 23. The 3D model of the eye outputted from the Mimics software from the front and lateral views. 


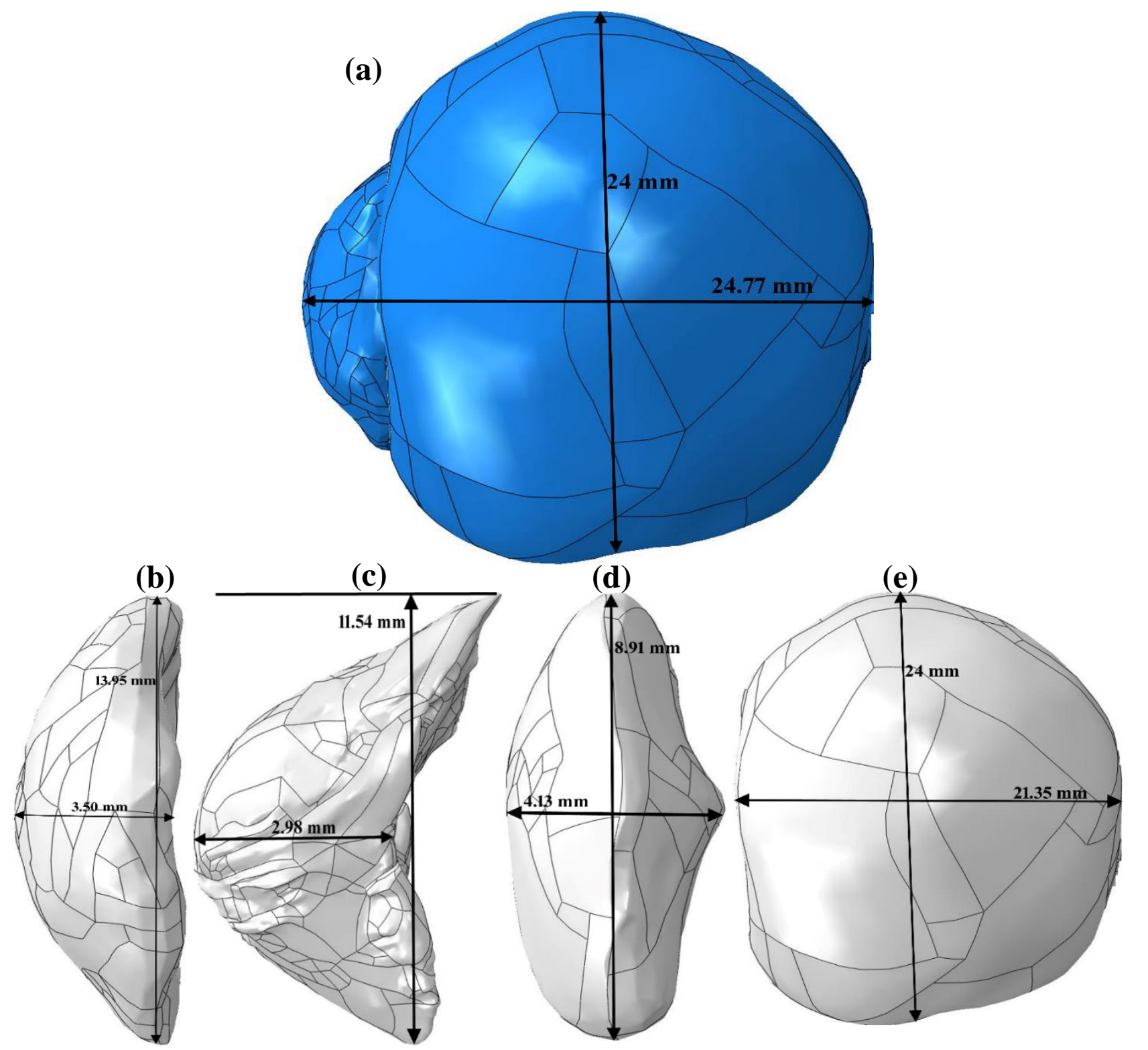

Fig. 24. The CT/MRI and FE models of the eye globe, including (a) the whole globe, (b) cornea, (c) iris/aqueous body, (d) lens, (e) sclera/retina/vitreous body. 

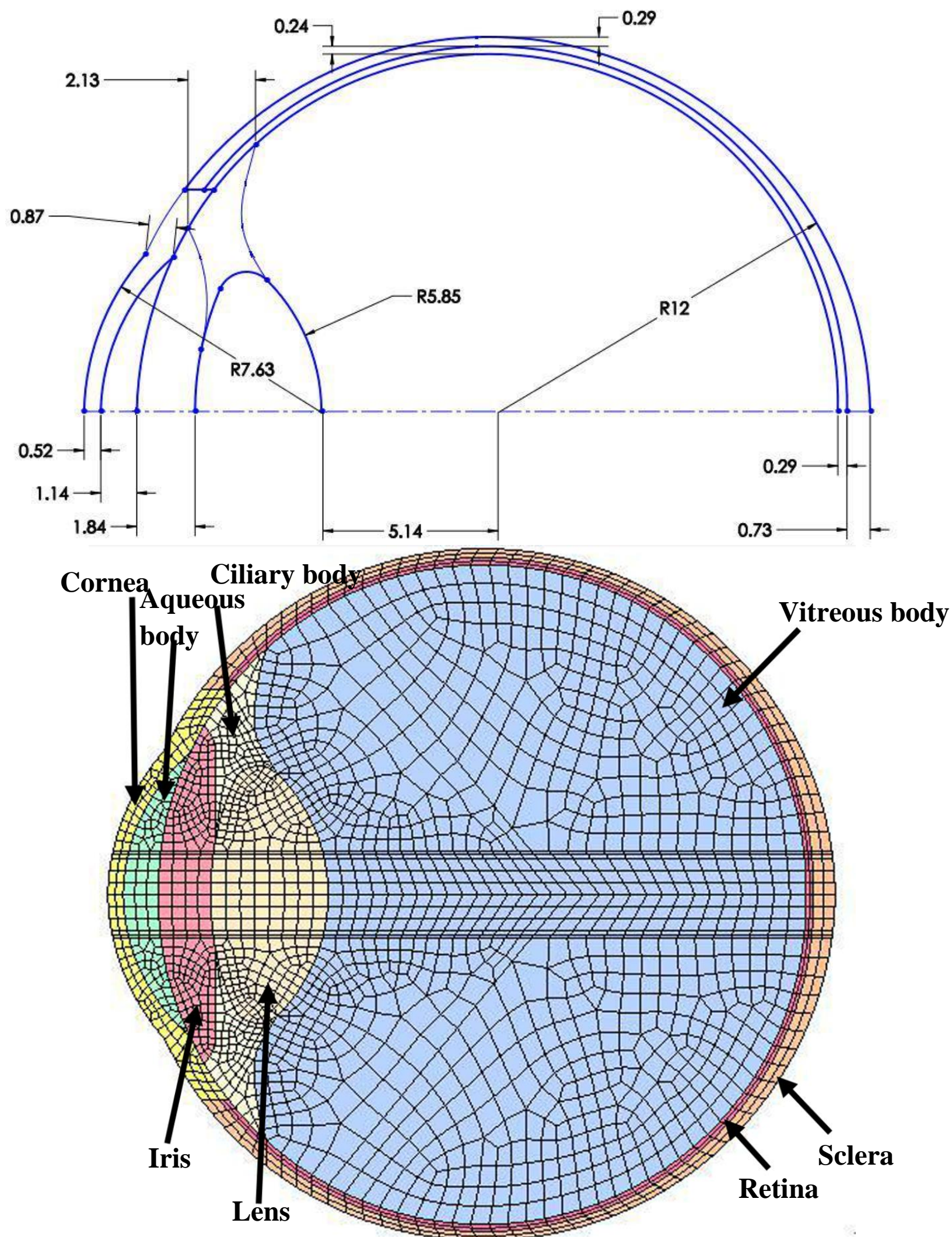

Fig. 25. The anatomical-based model of our own proposed eye model along with the meshed 3D form of the eye globe. 


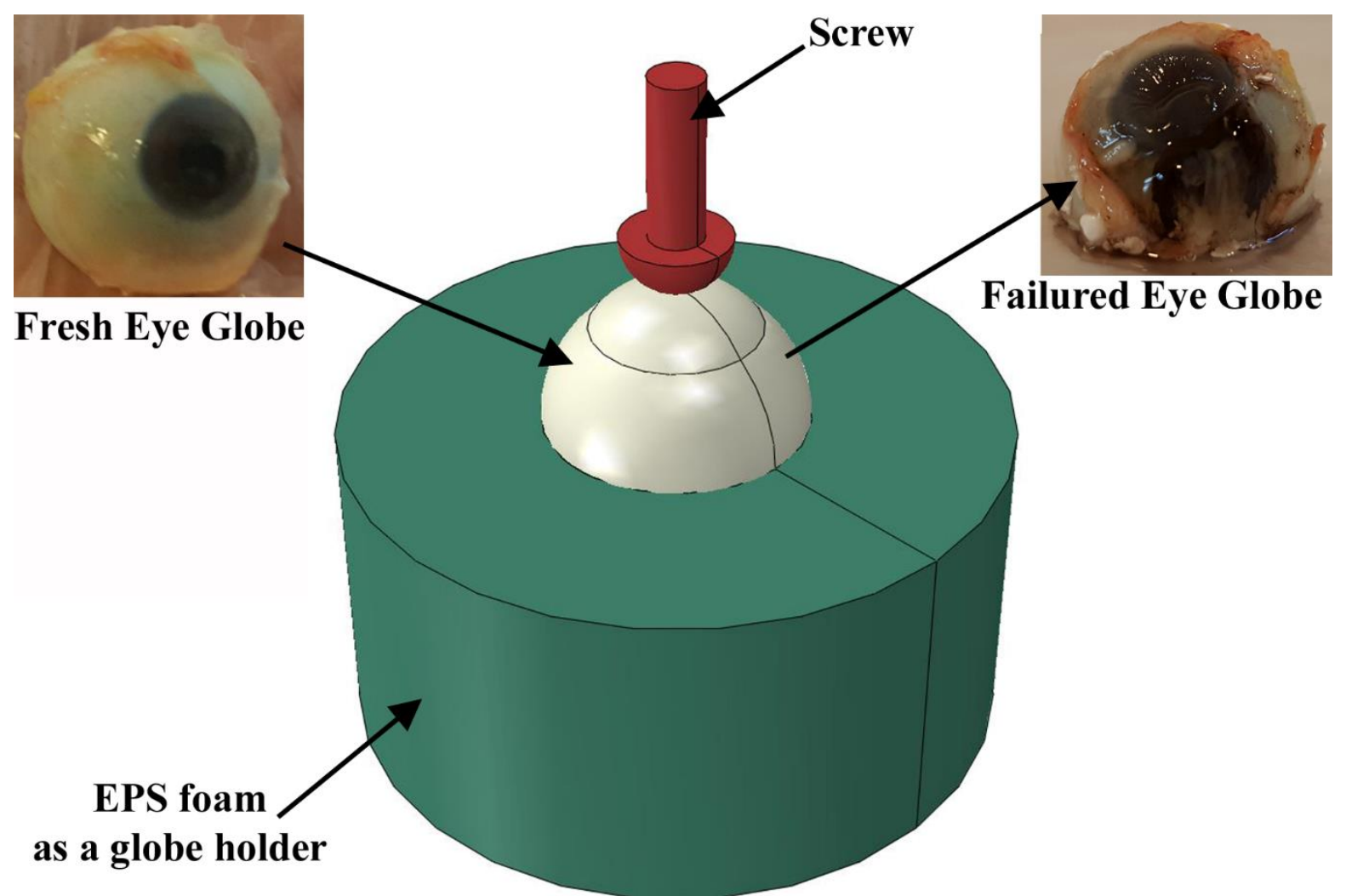

Fig. 26. A fresh eye globe was extracted from the human cadaver. Therefater, the globe was mounted inside a provided EPS and subjected to a loading at various rates using a fixed screw. The mechanically failured globe under the applied load. 

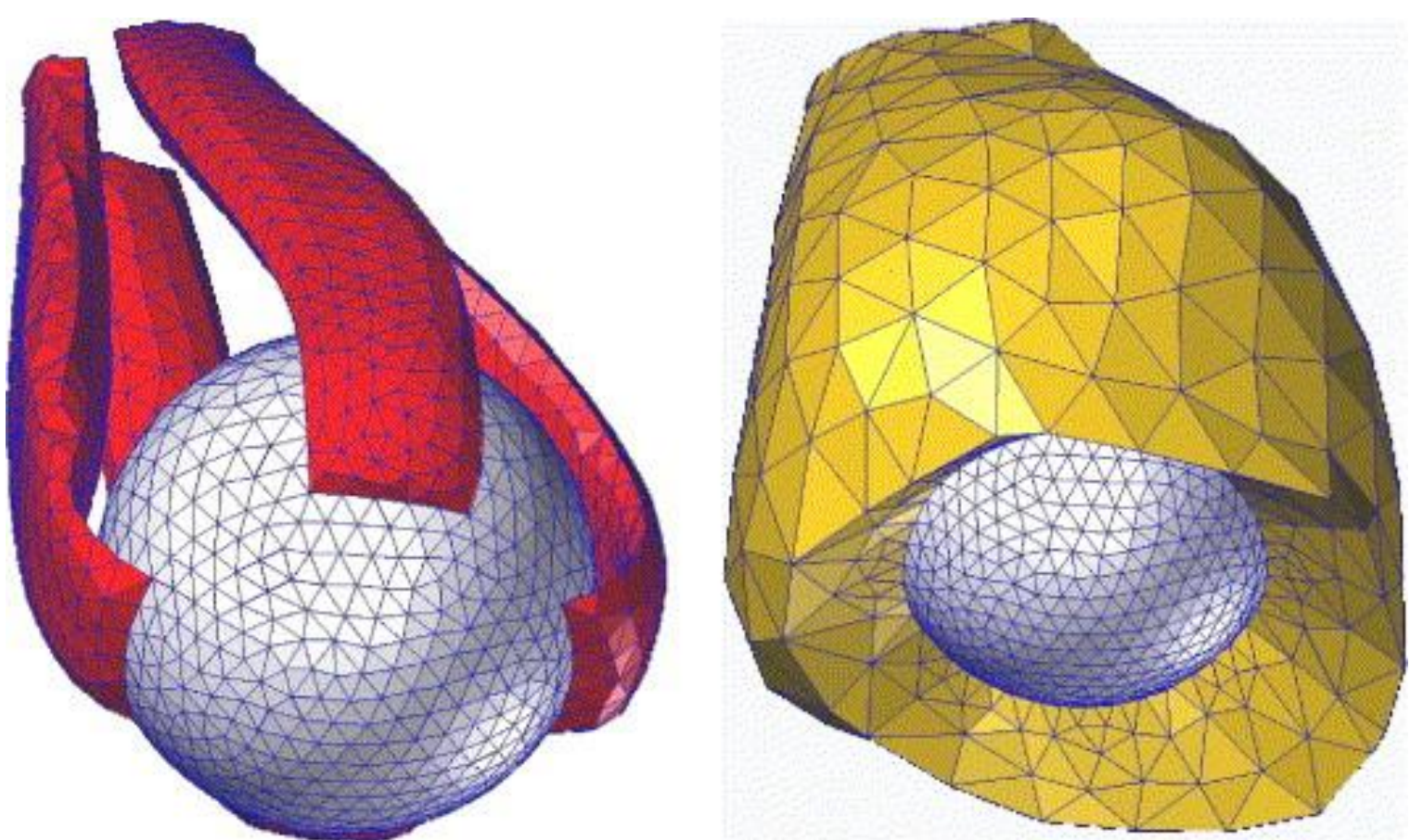

Fig. 27. The FE model of [57]. In this model, the globe was only modeled as an unique globe with single mechanical properties. License Number: 4034621357041. 


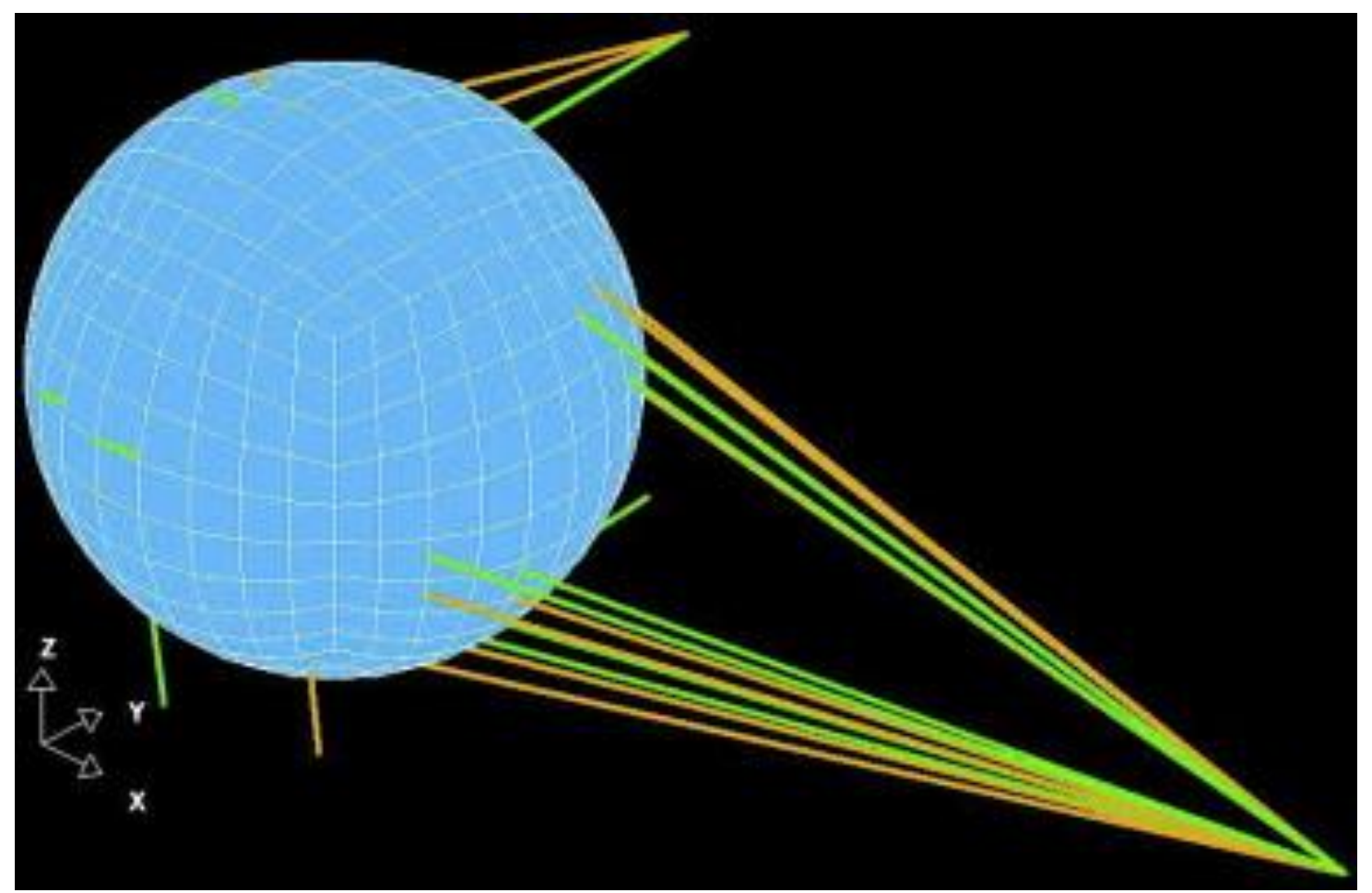

Fig. 28. The FE model of [58]. In this model, the eye globe was consisted of the cornea, sclera, vitreous body, and lens. License Number:4034621481812 

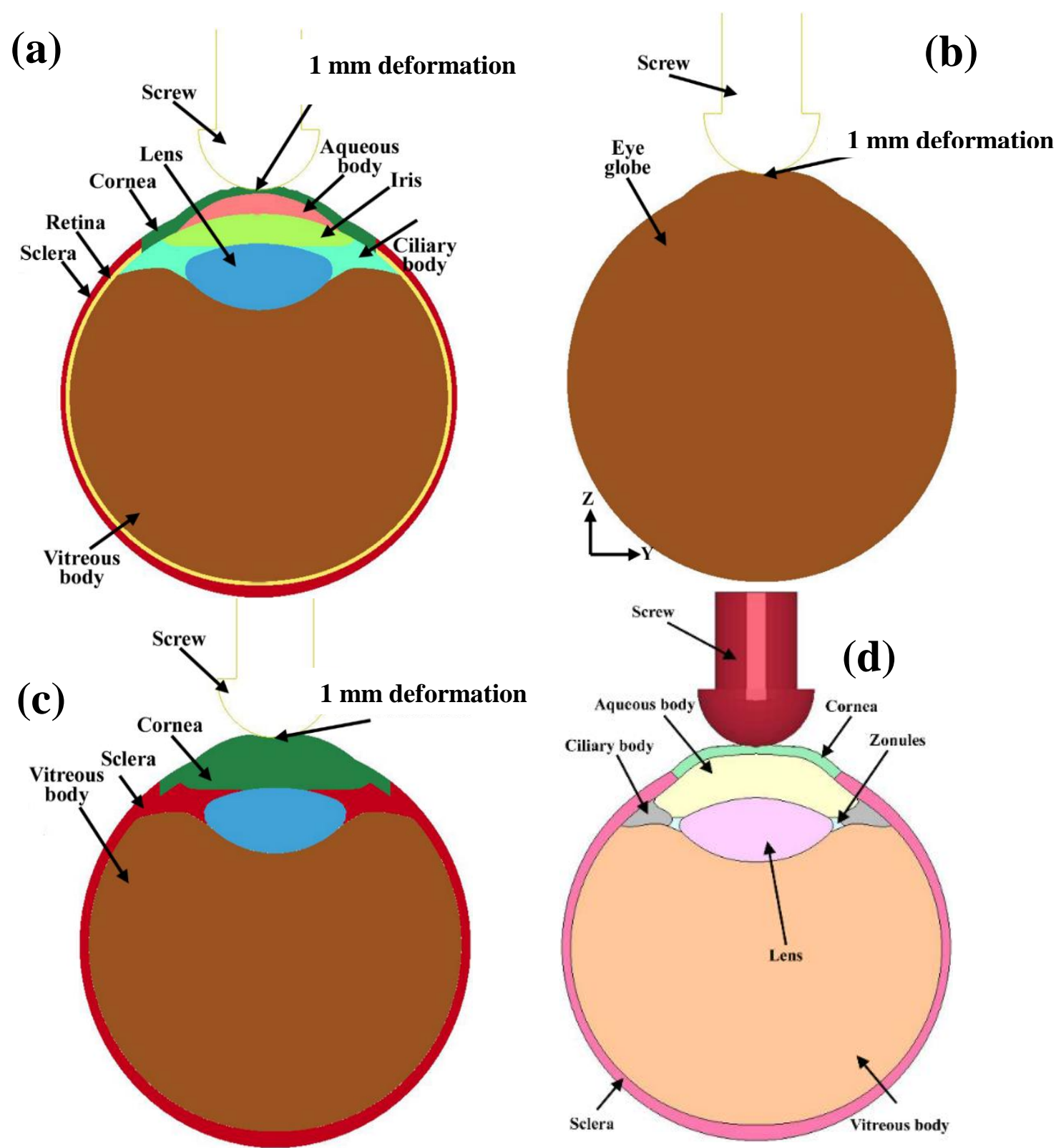

Fig. 29. The finite element models of the human eye. The MRI/CT data of the eye was obtained and employed to establish a solid surface of the eye from the DICOM images using MIMICS software. Four different models were selected as (a) our model, (b) $1^{\text {st }}$, (c) $2^{\text {nd }}$, and (d) $3^{\text {rd }}$ models. The cornea was subjected to a moving screw at 5 different strain rates, including 5, 10, 20, 50, and $100 \mathrm{~mm} / \mathrm{min}$. In order to simulate the experimental procedure and compare the results, the same process was simulated thru the established FE model. 


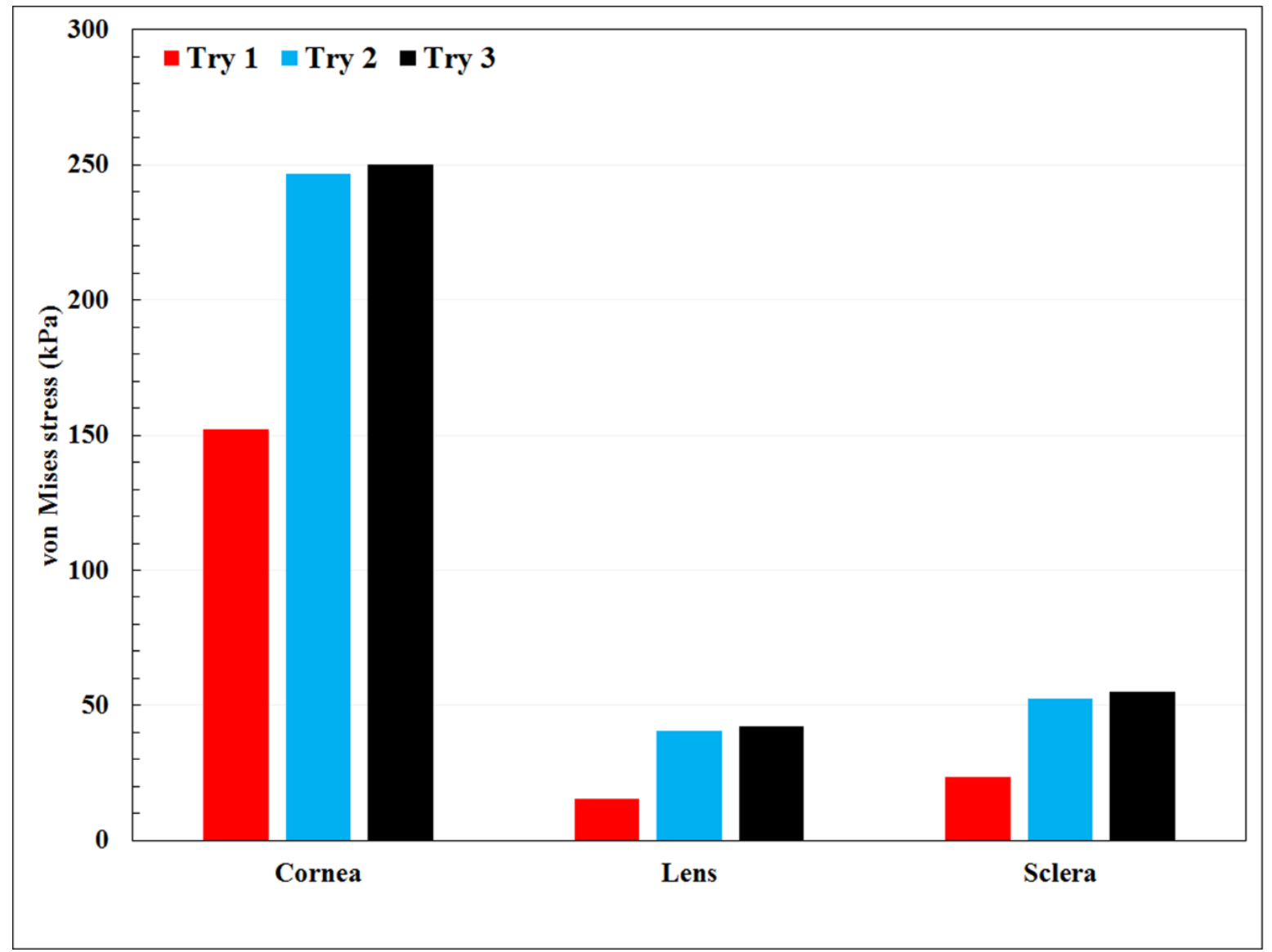

Fig. 30. The amount of von Mises stress at three different mesh densities for the cornea, lens, and sclera under the strain rate of $100 \mathrm{~mm} / \mathrm{min}$. 


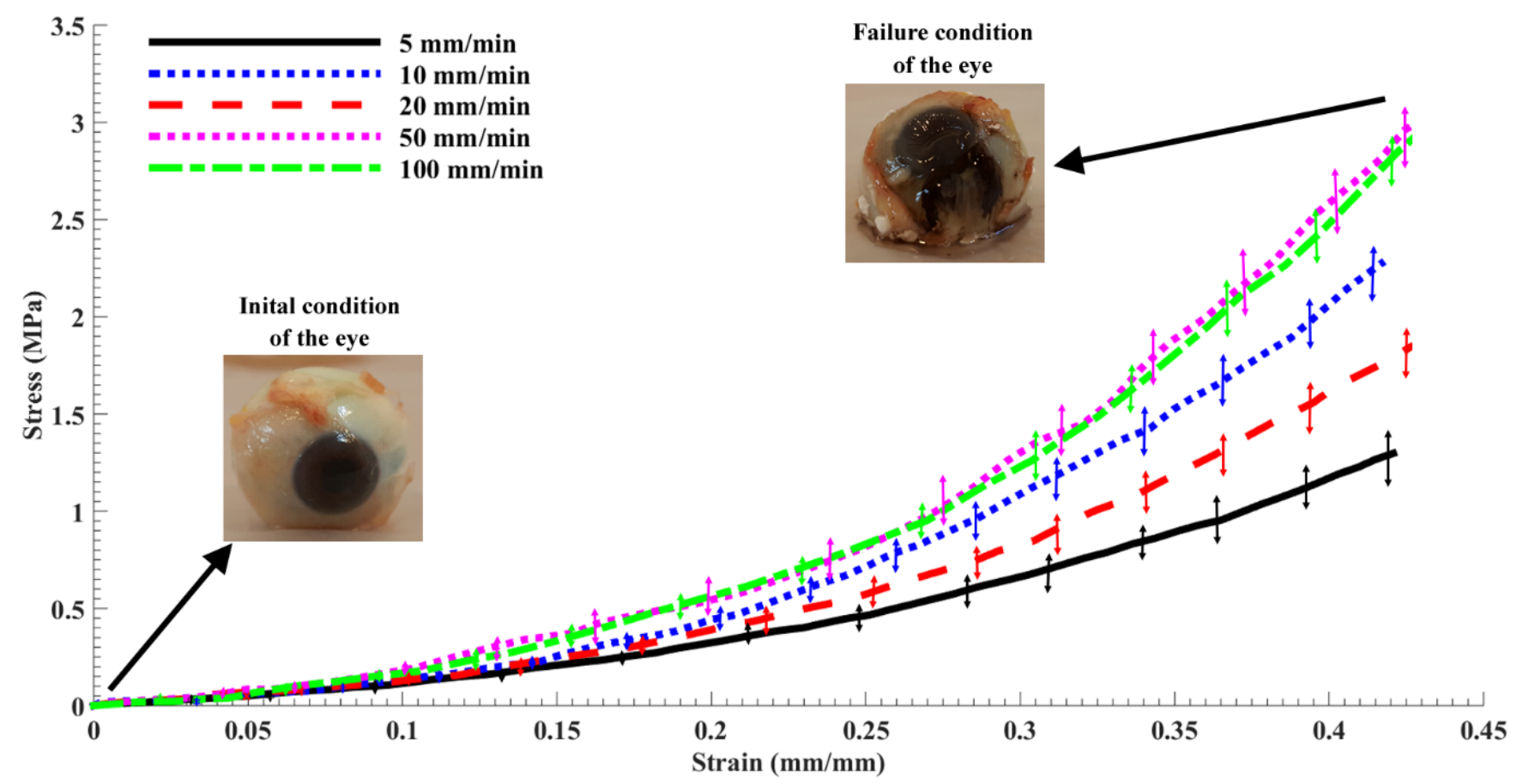

Fig. 31. The stress-strain diagrams of the eye globe under different strain rates.

Each curve is the mean representative of at least four curves and in here the mean one only is displayed. The condition of the eye globe both in the initial and failure conditions/configurations are also represented in the initial and final locations of the curves. 


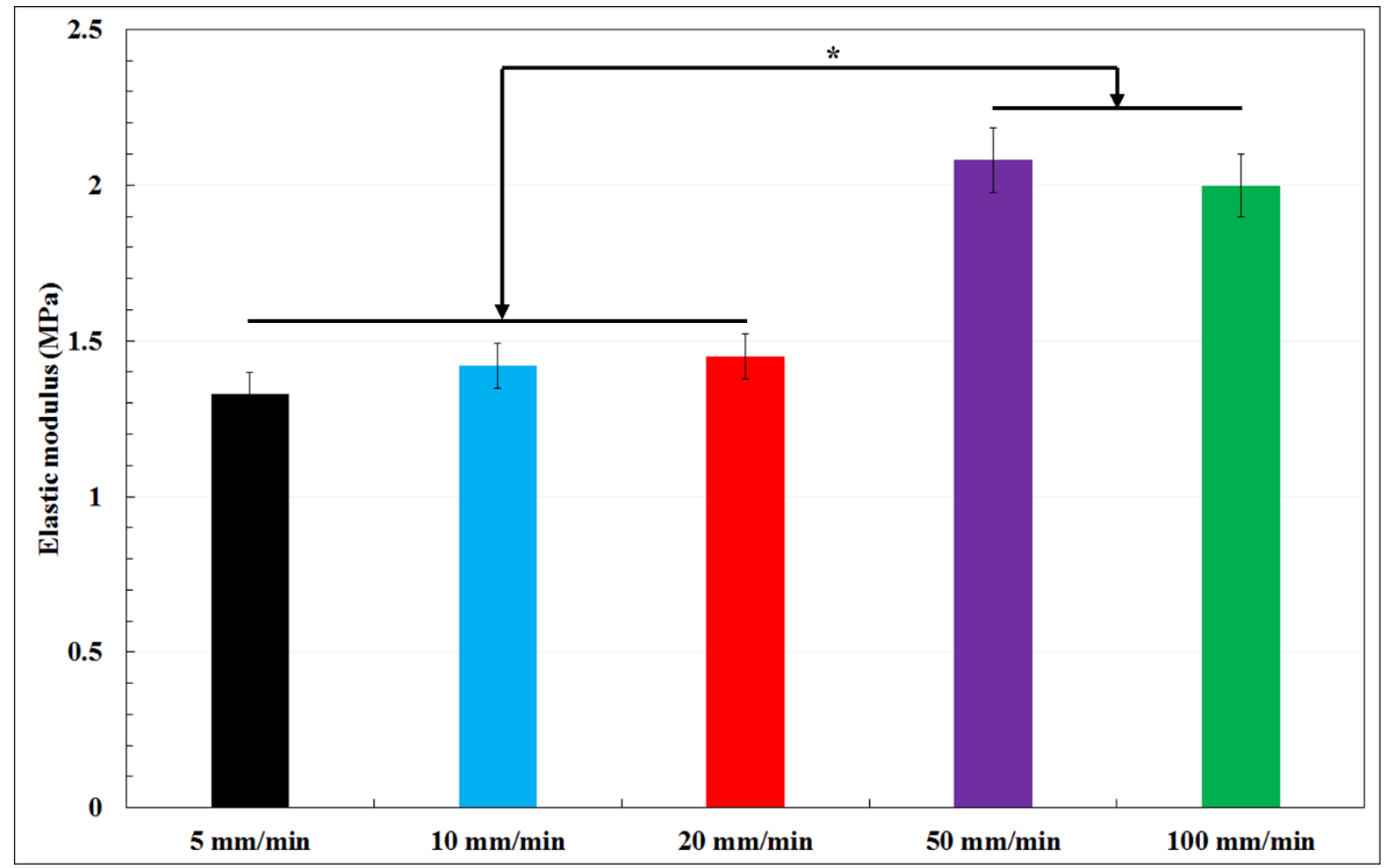

Fig. 32. The bar chart representation of the elastic modulus under different

strain rates for the eye globe. 


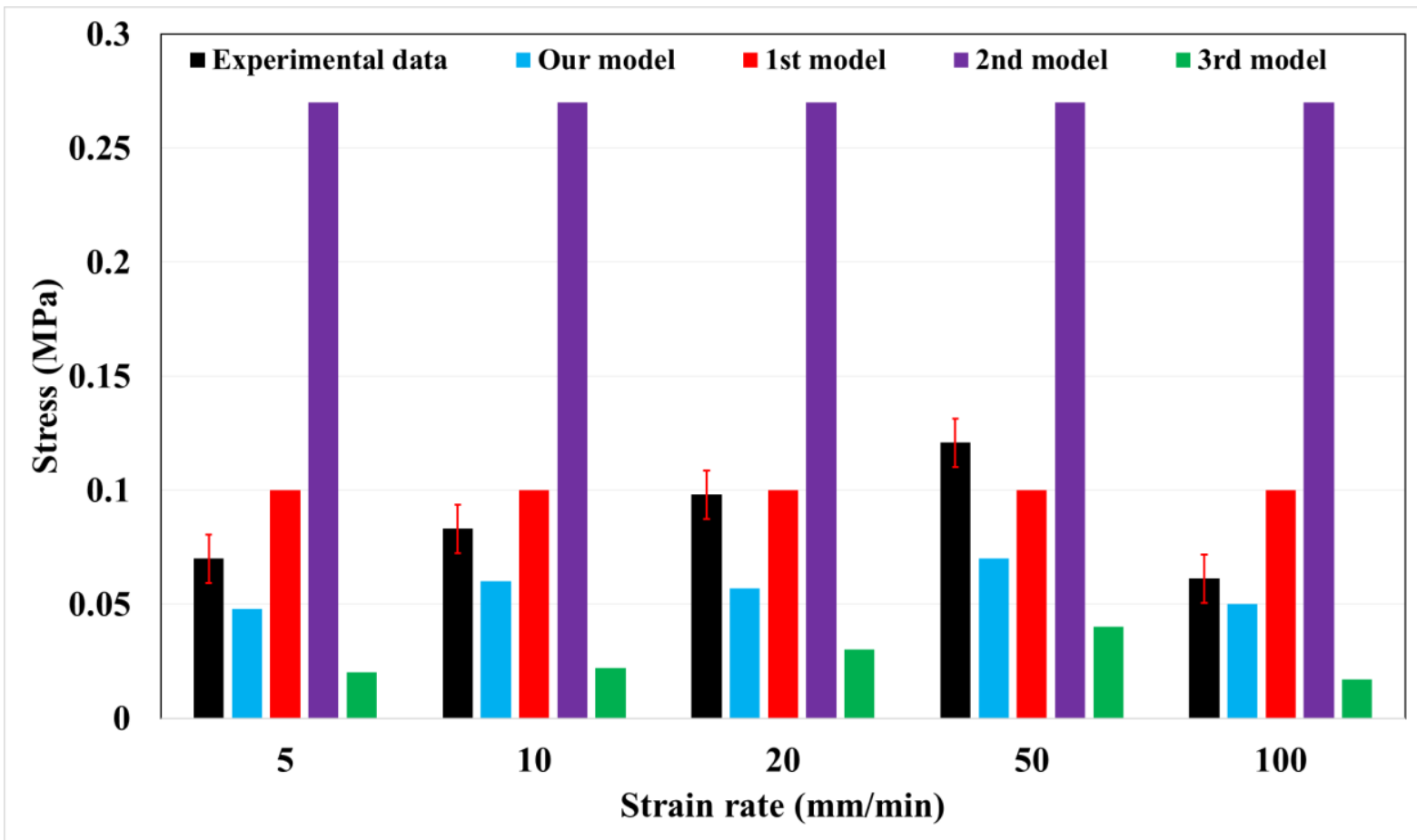

Fig. 33. The experimental stress results at various strain rates, including 5, 10, 20,50 , and $100 \mathrm{~mm} / \mathrm{min}$ up to the deformation of $1 \mathrm{~mm}$ in the cornea versus the numerically computed stresses of our model, $1^{\text {st }}, 2^{\text {nd }}$, and $3^{\text {rd }}$ models. 

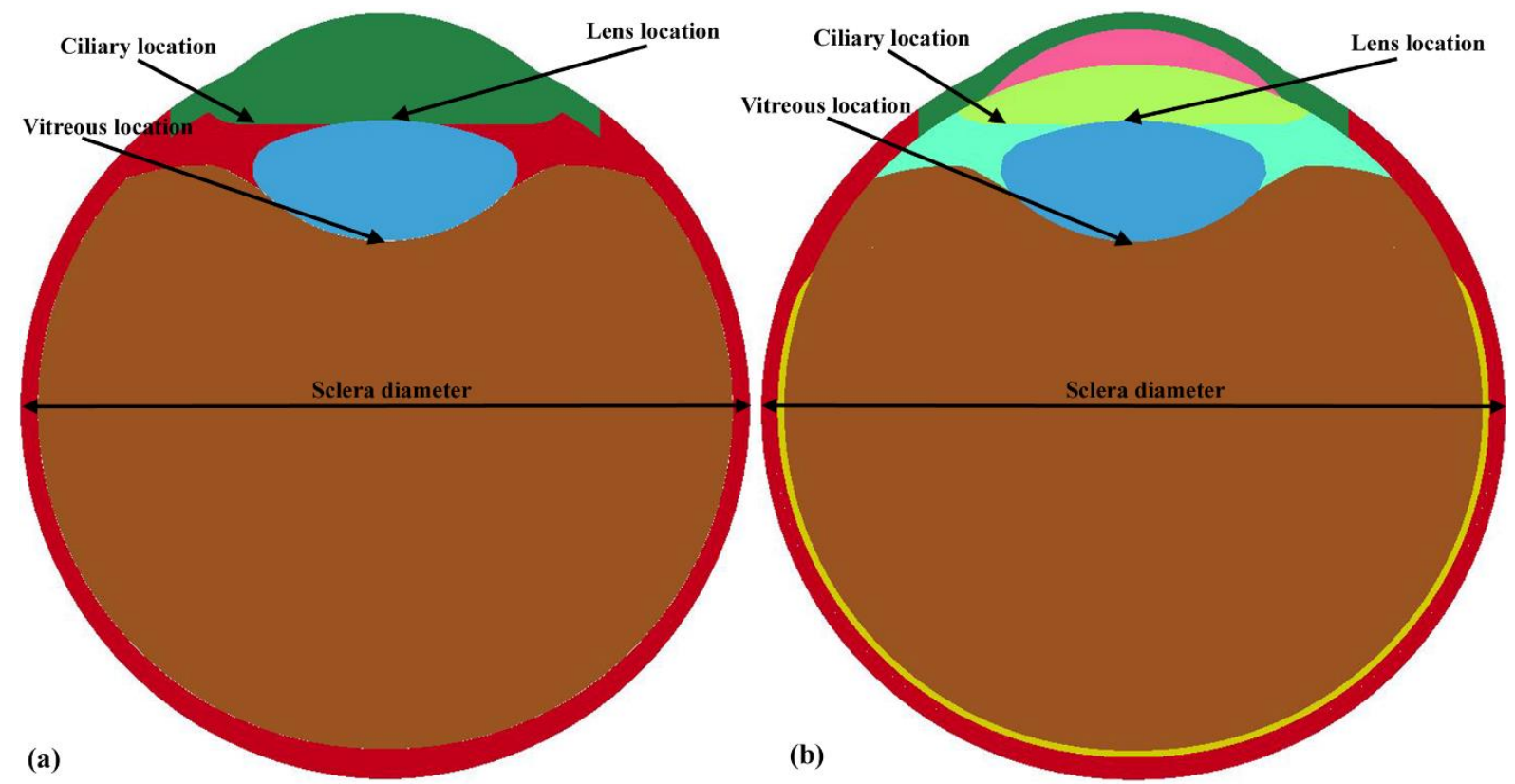

Fig. 34. The locations of the selected nodes/elements on the (a) $2^{\text {nd }}$ and (b) our models. The amount of stress and deformation in these points were calculated and compared. 


\section{Chapter 4 \\ Application of the \\ Anatomical-Based Eye \\ Model in the Anterior and \\ Posterior Injuries}


As it was pointed out, in this chapter it is aimed at understanding the amount of stresses and deformations in all the components of the eye as a consequence of anterior and posterior injuries to the eye components. In here, the anterior injury relates to the tennis ball impact which is a blunt trauma impact. And the posterior injury relates to the IOP alteration. Since it is experimentally not plausible to measure the amount of stress or deformation in all the components of the eye, especially the anterior and posterior sides, as a consequence of blunt trauma impact as well as IOP alteration, using the suitable numerical model of the eye is so useful. In addition, since so far almost all the human eye models ignore the role of iris and optic nerve in their models, the proposed model in this study has advantage as it enables to figure out the amount of stress/deformation as a result of tennis ball impact and IOP variation in all the components of the eye. On the one hand, tennis ball and IOP alteration invokes small deformation in all components of the eye. On the other hand, we found that our model shows a very good agreement with the experimental data even better than other three models of the eye under the small deformation. Therefore, the proposed model enables to accurately shed light on the effect of tennis ball impact as well as IOP alteration in all components of the eye in detail. We also considered the essential role of the sclera/optic nerve mechanical properties on the $\mathrm{ONH}$ biomechanics as a result of tennis ball impact as well as increasing the IOP to $30 \mathrm{mmHg}$ which is as twice as bigger than its normal range $(15 \mathrm{mmHg})$ in the eye. In the other words, we wanted to emphasize that first, the sclera has a pivotal role in load bearing conditions and, 
therefore, its mechanical properties are of great importance if a detailed accurate injury on the $\mathrm{ONH}$ is expected. In addition, we wanted to inform that among the variations in the range of the sclera's mechanical properties, the accurate mechanical properties of this tissue are needed since they can affect the deformation and stress of the $\mathrm{ONH}$ in its attachment site to the sclera.

\subsection{Anterior injury (Tennis ball impact)}

In this section, the mechanical stresses and deformations of all the eye components as a result of the tennis ball impact at the velocity of $69.29 \mathrm{~m} / \mathrm{s}$ were investigated. The injury in this section is related to the anterior side of the eye although the posterior components of the eye also affected. However, since the tennis ball impact is the blunt trauma impact, therefore, the most part of the injury must be located in the anterior side of the eye. In the following subsections, the process of numerical simulation is explained.

\subsubsection{Model explanation}

Computational modeling of the human eye according to the FSI model was performed to anticipate the structure of mechanical response of the components under the impact of the tennis ball. The meshed structure model of the human eye is displayed in Fig. 35. As it is observable, in the current model the optic nerve, intra and extraconal fat as well as the muscle are added. The morphology of the 
optic nerve was obtained from our own CT/MRI data. In addition, the attachment site of the muscles' head to the eye globe was determined using the CT/MRI data. However, since the final structure of the muscle was not suitable in other parts, its dimensions were extracted from the literature. Furthermore, the structure of the intra and extraconal fats were incorporated into the model according to the literature data [57]. The process of load applying as well as the boundary condition of the tennis ball impact model is displayed in Fig. 36.

The CT/MRI data for the head with the special focus on the intra and extraconal fats as well as the muscles are provided in Fig. 37 . In addition, the highlighted optic nerve as well as the muscles' head are presented in Fig. 38.

Since in chapter 4 it was aimed at focusing on the amount of stress and deformation of the $\mathrm{ONH}$, the structure and attachment site of the optic nerve to the sclera specifically is presented in Fig. 39. The mechanical properties of these components are also listed in Table 4. The mechanical properties of the sclera and the optic nerve were the same. The reason why we have not considered these components in our previous simulation was that, since we wanted to perform a comparative study among other eye models only the globe by itself was modeled. However, in here since the role of optic nerve properties and its attachment site to the sclera want to be investigated, these parameters were added into the eye model (Figs. 35 and 39). The mechanical behavior of the corneal tissue assumed to be approximately incompressible as well as linear elastic. Indeed, as the deformation of the cornea in the eye would not profoundly exceed to the applied 
load, its deformation can be considered as small deformation and, as a result, linear elastic [61]. The mechanical properties of the iris in our published paper was Ogden hyperelastic with the coefficients of $\mu_{1}=43.05 \mathrm{kPa}, \mu_{2}=37.70 \mathrm{kPa}$, $\alpha_{1}=54.25, \alpha_{2}=48.07$ [76], and in the current part of the manuscript the iris was considered to be elastic. Therefore, we performed a comparative analysis to show that there is no difference in the stress of the other components as a result of this variation in the sclera/optic nerve mechanical properties and its results in terms of the stress in all the components of the eye are listed in Table 10. For doing that, the eye model subjected to the tennis ball impact at the velocity of $69.29 \mathrm{~m} / \mathrm{s}$ and the resulted stresses were calculated and reported. Since the results in this regard showed no considerable differences, in the rest of the simulation results in this chapter, the iris was considered to behaved as an elastic material. The amount of stresses and deformations in the components of the eye in here were reported as a result of $1 \mathrm{~mm}$ deformation in the apex of the cornea.

Since there were two different elastic modulus for the sclera/optic nerve tissues, in order to have a comparative assessment on the role of the elastic modulus on the amount of stresses and deformation of the eye components, two different elastic moduli were assigned to the model and the results were calculated and compared. One was reference data with 5.50 MPa and one was our own data with $1.65 \mathrm{MPa}$ (the average elastic modulus of the sclera). Therefore, in the following subsections, the results are reported for two different elastic moduli of the sclera/optic nerve. 


\subsubsection{Results}

In the current study the injury to the human eye due to the tennis ball impact was modeled using dynamic FE simulation code, namely LS-DYNA. The process of modeling was defined as a tennis ball at a speed of $69.29 \mathrm{~m} / \mathrm{s}$, which is the record of the highest speed of a tennis ball so far in an official Association of Tennis Professionals (ATP) game, was hit the anterior side of the eye and then the injury in terms of the stresses and deformations were computed and reported.

The type of injury here we are facing is the blunt injury. In this type of injury an object, here a tennis ball, causes sudden compression of the eye. Bleeding in the front of the eye between the cornea and iris which cannot be seen by FE models is the common results of more severe blunt trauma. Severe blunt trauma may lead to the retina or optic nerve damage which may invoke permanent visual loss [77]. The contours of stress distribution in the retina and sclera are illustrated in Fig. 40. The results for the retina at two different elastic modulus of 1.65 and 5.50 MPa showed the values of 5.31 and $7.58 \mathrm{kPa}$, respectively. In addition, the stress values of 8.29 and $32.51 \mathrm{kPa}$ were observed in the sclera at the elastic modulus of 1.65 and $5.50 \mathrm{MPa}$, respectively. The optics of the eye is responsible to create an image of an object on the retina via the cornea and lens. The retina is also considered as a layered structure of neurons interconnected by synapses. The sclera is consisted of collagen and elastic fiber. It also holds the posterior $5 / 6$ of the connective tissue cover the globe. It is also continuous with the dura mater and the cornea, and is in charge of maintaining the shape of the globe, offering 
resistance to internal and external forces. The values of stresses in the sclera imply that the stiffer sclera overestimate the resulted stress in the wall of the tissue.

The sclera is also perforated by many nerves and vessels passing through the posterior scleral foramen, the hole that is formed by the optic nerve [78]. Therefore, any values of stresses and deformations which affect the performance of these two layers of the eye would trigger visual acuity impairment. That is, the stresses induced due to the tennis ball impact might dislocate the structured layers of the retina or lead to rupture in the collagen and elastin fibers of the sclera and, as a result, it would invoke vision impairment. A bruise retina can occur from blunt trauma. It actually happens when the eye transmitted the force of the tennis ball blow to the retina causing swelling. This usually disappears although if severe can lead to scarring and some loss of vision at that site. If this occurs in the area responsible for the macula, it can result in permanent vision loss. The stress induced in the retina due to the tennis ball impact was lower than that of the elastic limit of the retina $(20 \mathrm{kPa})$ which assure no serious injury in the special case of tennis, however, the occurrence of minor injury in the retinal wall is undeniable. Retinal damage, retinal breaks, or retinal detachment can occur sometimes after the blunt injury [77].

A comparative histogram representation of the von Mises stress in different eye components, including vitreous body, extra and intraconal fats, optic nerve, and aqueous body at two different elastic moduli is exhibited in Fig. 41. The vitreous body in this group indicated the lowest amount of stress with $2.14 \mathrm{~Pa}$ 
while the aqueous body shows the highest one with $260.1 \mathrm{~Pa}$. The intraconal fat also experienced a higher amount of stress compared to the extraconal one. Different elastic modulus for the sclera/optic nerve in here showed no difference. The composition of the vitreous body is similar to that of the cornea, yet the number of cells in the vitreous is smaller than the cornea. Thus, the stress in this pretty delicate material would bring about severe injury and then visual acuity impairment. The intraconal orbital fat is embedded in the anterior and is formed by the posterior half of the globe. Since the intraconal fat holds the optic nerves and ophthalmic arteries, it is so vulnerable to stresses and deformations. The optic nerve is a paired nerve that conveys visual information from the retina to the brain [79]. The optic nerve is derived from optic stalks during the seventh week of development and is composed of retinal ganglion cell axons and glial cells. The numerical results showed the stress value of $230.3 \mathrm{kPa}$ which might subject the performance of the whole eye vision into problem. The aqueous humor is a gelatinous fluid similar to plasma. It is secreted from the ciliary epithelium, a structure supporting the lens. It is located in the space between the lens and the cornea. Compression of the globe in an anterior-posterior direction due to the blunt loading results in the equator of the globe to bulge in a coronal plane. This inward-directed tractional force on the retina by the vitreous can triggers a retinal dialysis, yet it can also invoke retinal tears and vitreous hemorrhage as retinal vessels may be ruptured. Although the proposed model was unable to simulate this phenomena, however, it could happen in the interaction site of the retina- 
sclera as a result of tennis ball impact. In addition, as the stress in the vitreous body only induced at the anterior center side, hence, the interaction of the retina with vitreous body at least during the impact time has no role in the amount of the stress induced in the vitreous body. Therefore, in the tennis most concentration of the injury/stress would be in the anterior side of the eye and actually this amount of stress due to the other relatively stiff components would be damped and vitreous would not be seriously damaged. In addition, the vitreous body only has interaction with retina from the posterior side and lens as well as ciliary body from the anterior side. Furthermore, as the main source of the traction force was in front of the eye, as a result, the main source of the stress inducement must be in the anterior side of the eye. It should be noted that a low amount of stress in the vitreous body might be considered as a sign of posterior vitreous detachment which is usual in blunt injuries, especially sudden impact, which has been proven in other research. Regarding the optic nerve, our results are in agreement with the previous study which reported a very low rate of injury for the optic nerve after blunt injury [77].

\subsubsection{Discussions}

The values of von Mises stress on the muscle, iris, lens, and ciliary body are demonstrated in Fig. 42. The ciliary body experienced the stress of $14.85 \mathrm{kPa}$ while the iris, lens, and muscle depicted the stress of $2.28,10.67$, and $1.72 \mathrm{kPa}$, respectively. Different elastic modulus for the sclera/optic nerve in here showed 
almost no difference. Four of the extraocular muscles control the movement of the eye in the four cardinal directions: up, down, left and right. The remaining two muscles control the adjustments involved in counteracting head movement. The actions of the six muscles responsible for eye movement depend on the position of the eye at the time of muscle contraction. The lens is a biconvex component in the eye provides light refraction in a way to focus on the retina [80]. The lens enables to alter its shape to change the focal distance of the eye in order to focus on objects at various distances, therefore, letting a sharp natural image of the object to be consisted on the retina [81]. The ciliary body is attached to the lens by connective tissue called the zonular fibers. Relaxation of the ciliary muscle also puts tension on these fibers and changes the shape of the lens in order to focus light on the retina [82]. The stresses on the muscle, lens, and iris would also affect the quality of vision by changing the structure of the eye components. For example, the stress on the lens would lead to shape deformation from its normal biconcave shape. Since the lens by changing its shape provides vision at various distances, any deformation in this material would alter its performance. In addition, since the ciliary body acts as a holder of the lens in the eye, a relatively high amount of stress in that would be related to the stress concentration in its contact region with the lens as well as the retina.

The cornea has unmyelinated nerve endings sensitive to touch, temperature and chemicals; a touch of the cornea causes an involuntary reflex to close the eyelid. The cornea has no blood supply; it gets oxygen directly through the air. 
Oxygen first dissolves in the tears and then diffuses throughout the cornea to keep it healthy. It borders with the sclera by the corneal limbus [83]. Blunt trauma may lead to blood vessel rupture related to the iris and ciliary body, producing hyphema and spillover hemorrhage into vitreous. The lens damage would also lead to cataract formation, lens subluxation or dislocation which may happen due to a high amount of stress in this component [77]. Rupture of the globe was also reported as a result of a very severe blunt injury to the eye from a blunt trauma. The eye contents prolapse through the weakest part of the eye wall, causing an open globe injury [77].

The variation of radius of curvature for the cornea and lens were also calculated and presented in Fig. 43. The results revealed that until the time of 7 $\mu$ s no alteration was observed for the cornea and lens during the collision of the tennis ball, however, right after that the radius of curvature of the cornea going to decrease in about $0.0003 \mu \mathrm{m}$. In contrast, the radius of curvature of the lens is going to increase by the passage of simulation time in about $0.055 \mu \mathrm{m}$. The resultant displacement of the optic nerve after impact was also computed and it showed the value of $0.045 \mu \mathrm{m}$ (Fig. 44). This amount of displacement is not significant and may not invoke injury to the eye nerve.

The results of this study suggest that those eye components in the anterior side of the eye would be more injured compared to the posterior side due to the impact by the tennis ball $[84,85]$. In order to minimize this injury, the eye protection can be considered in the concept of eye protection [86]. The value of certain eye 
guards has been established in several sports, and our data strongly support their use to minimize the injury [87]. Although the presented model and its material properties contains a number of limitations, such as considering an isotropic mechanical behavior of the materials of the model, we tried to make the model more applicable by adding all the eye components to the eye. Some of these materials, such as ciliary body, sclera, retina, and cornea, in spite of the fact that their mechanical properties have not been measured deeply, may show nonlinear mechanical behavior with hyperelasticity which should be taken into account in the future simulation. In addition, as previously pointed out, the eye model was established on a basis of CT images in order to reach to a model as close as possible to the real condition of the human eye, however, as it was too difficult to distinguish some parts of the eye as well as their boundaries, the authors simplified some parts of the model. For example, the shape of the optic nerve was considered to be a hollow cylinder which is horizontally attached to the posterior side of the macula. In a real anatomical structure of the eye, the macula is not sited at the optic nerve head as indicated in Fig. 44, but approximately 20 degrees laterally to this structure. This might lead to inaccurate results around the optic nerve and other components of the eye, however, this is the authors' belief that the results of this study are still promising and further research in this area are required. These simplifications may hinder us to find the best counter of stress and strain in the eye components that should be addressed in future research. Furthermore, the orbital bone around the globe was not considered in the model, 
however, since the exterior side of the extraconal fat was act like a solid object (boundary condition of the front side) it could be considered as a bone. However, this is the authors' belief that the results of this study are still promising and further research in this area are required. These simplifications may hinder us to find the best counter of stress and strain in the eye components that should be addressed in the future research.

\subsection{IOP alteration}

In this chapter it is aimed at stimulating the injury to the posterior side of the eye as a result of IOP variation, including the 10, 20, and $30 \mathrm{mmHg}$.

\subsubsection{Clinical explanation}

The optic nerve in the posterior side of the eye globe has a pivotal role by transferring the electrical signals to the brain [28]. IOP is defined as the intra pressure between the cornea and iris in the anterior side of the eye $[29,88]$. Its variation can substantially affect the shape of the cornea and, as a result, alter the visual acuity of the eye [89] following by a blurred vision [31]. Keratoconus, which is defined as an anti-inflammatory corneal disorder, occurs as a result of increasing in the amount of IOP in the weakened cornea $[90,91]$. Normal IOP values in the aqueous have been reported as $15.60 \pm 2.70 \mathrm{mmHg}$ [92]. In addition, it has been reported that the cornea behaves linearly elastic in the range of 10 to 
$30 \mathrm{mmHg}$ [93]. Therefore, in this type of simulation considering the linear elastic behavior for the components of the eye is an acceptable assumption.

The mechanical properties of the sclera because of its connection to the optic nerve are deemed important. The optic nerve in the numerical simulations also was considered to behave same as the sclera's mechanical behavior and this is why the mechanical properties of the sclera should be chosen accurately if correct stress and deformation calculation in the $\mathrm{ONH}$ site are expected.

\subsubsection{Model explanation}

Computational modeling of the human eye according to the FSI model was performed to anticipate the structure of mechanical response of the components under three different IOPs, including 10, 20, and $30 \mathrm{mmHg}$, to understand the precise variation of the eye components' deformation and stress as the function of IOP. The process of load applying as well as the boundary condition of the model are displayed in Fig. 45. The mechanical properties of these components are also listed in Table 5. The mechanical properties of the sclera and the optic nerve were the same. The reason why we have not considered these components in our previous simulation was that, since the we wanted to perform a comparative study among other eye models only the globe by itself was modeled. However, in here since the role of optic nerve properties and its attachment site to the sclera want to be investigated, these parameters were added into the eye model. 
Deformation of the cornea under various IOPs $(10,20$, and $30 \mathrm{mmHg})$ were considered to be comparatively minor in comparison with the cornea's radius of curvature $[29,94]$. Therefore, the mechanical behavior of the corneal tissue assumed to be approximately incompressible as well as linear elastic. Indeed, as the deformation of the cornea in the eye would not profoundly exceed to the applied load, its deformation can be considered as small deformation and, as a result, linear elastic [61]. The mechanical properties of the eye components for this simulation are listed in Table 5 [76]. The same comparative simulation regarding the role of the iris nonlinearity in the results at the IOP of $30 \mathrm{mmHg}$ was carried out and its comparative results were listed in Table 11.

The deformation of the cornea through the analysis was defined as it fixed at the sclera, and forced to have a radial motion according to the previous experimental observations [93]. Normal IOP values in the aqueous have been reported as $15.60 \pm 2.70 \mathrm{mmHg}$ [92]. In addition, as it has been reported that the linear elastic behavior of the corneal tissue is just bounded between 10 to 30 $\mathrm{mmHg}$, the cornea was endangered to the IOP of 10,20 , and $30 \mathrm{mmHg}$ with the name of small, moderate, and high [93]. A total of $1240 \mu$ s were executed for simulation with a time step of $10 \mu \mathrm{s}$. The IOP starts to apply on the components which are in the direct contact with the aqueous body as the pressure with the $\Delta \mathrm{P}$ of $0.0806,0.1612,0.2419 \mathrm{mmHg}$ at each time step up to 10,20 , and $30 \mathrm{mmHg}$, respectively, was increased. 


\subsubsection{Results}

\subsubsection{IOP variation results}

This study benefitted from a dynamic FE code to investigate the stresses and deformations in the human eye components due to IOP variations. To do this, three different IOPs, including 10, 20, and $30 \mathrm{mmHg}$, were imposed in the aqueous and its effects on the other components of the human eye were assessed in terms of the stresses and deformations. A comparative bar chart representation of the highest von Mises stress in the (a) cornea and (b) lens at various IOPs is indicated in Fig. 46.

The distribution of principal stresses through the center of the cornea indicates the peak stress value of $247.25 \mathrm{kPa}$ in the corneal apex at the IOP of $30 \mathrm{mmHg}$. The highest stress on the lens was also observed at the IOP of $30 \mathrm{mmHg}$ with $19.10 \mathrm{kPa}$. Although these stress values seem to be high to alter the shape and structure of the cornea and lens, none of them even at the IOP of $30 \mathrm{mmHg}$ triggers damage to the cornea or lens by exceeding the ultimate stress of these eye components. The stress in the cornea showed no substantial variance at two different pressures of 20 and $30 \mathrm{mmHg}$. As it pointed out the average value of the IOP is $15 \mathrm{mmHg}$ and the pressure over this value (between $20-30 \mathrm{mmHg}$ ) have almost the same effects on the other eye components. In contrast, the variation of IOP had influence on the amount of stress induced in the lens in a way that the stress at the IOP of $30 \mathrm{mmHg}$ is considerable $(46.15 \%)$ higher than that of the IOP of $20 \mathrm{mmHg}$. The radius of curvature for both the cornea and lens at various 
range of IOPs were calculated and presented in Fig. 47. Notably, an extensively slanted, swelled structure of the cornea was plainly visible in all the simulation cases. It verifies that the cornea owing to IOP is bulged outward and became conical while lens bulged inward. In addition, the highest amount of alteration in the radius of curvature of the cornea $(7.89 \mathrm{~mm})$ was observed at the IOP of 30 $\mathrm{mmHg}$, which as a result would lead to a conical shape of the cornea. This results is in good agreement with the clinical observations in this regard [95, 96]. Similarly, the alteration of the lens radius of curvature was invoked an inward shape for the lens which is in good agreement with the experimental observations which discussed the morphological characteristics of the lens due to IOP variation [97].

The highest radius of curvature for the lens was seen at the IOP of $30 \mathrm{mmHg}$ as well with $2.5 \mathrm{~mm}$ inward. As it has been mentioned that, even a small variation in the curvature of the cornea or lens would bring about blurred vision on the grounds of focusing deterioration. Therefore, the results well confirmed that only if the IOP is in the range of $10-15 \mathrm{mmHg}$ there would be no considerable alteration in the curvature and, consequently, there would be no eye vision impairment. Measurements of the radius of corneal and lens curvatures were made using three points on the corneal surface according to the literature [98].

The value of von Mises stress in the other components of the eye was also computed. Fig. 48 indicates the stress distribution in the iris. The highest stress in the aqueous body was found to be $32.43 \mathrm{kPa}$ at the IOP of $20 \mathrm{mmHg}$ while the 
lowest one was observed at the IOP of $10 \mathrm{mmHg}$ with $9.24 \mathrm{kPa}$. The stress distribution in the iris was asymmetry which is related to the toric structure of the lens and the pressure that cannot be applied directly on its surface. In addition, the distribution of the von Mises stress in the vitreous body is shown in Fig. 49. Vitreous was considered to behave like a viscoelastic material and the modeling result showed that there is no considerable influence regarding the alteration of the IOP on the amount of stress in the vitreous body.

A comparative bar chart representation of the von Mises stress in the (a) retina and (b) sclera at various IOPs are illustrated in Fig. 50. The results revealed that there will be a higher stress at a higher amount of IOP in retina, while by increasing the IOP there will be a decreasing of stress in the sclera. The reason might be related to the role of sclera in the eye which acts as a holder of eye components and any alteration in the pressure would change its stress. In addition, it has been reported that not only the IOP but also the radius of curvature of the sclera increases as the pressure volume increasing. There is no correlation between the elastic moduli of the cornea and sclera and that of the IOP; and the elastic modulus of the sclera is higher than that of the cornea. Hence, it is expected that the elevation of IOP would lead to the alteration of the curvature of the sclera but not that of the cornea [94]. In addition to that, as it is observable that the variation of the von Mises stress in the retina and sclera at various IOPs are not significant as there are only 0.5 and $1.65 \mathrm{kPa}$ difference between the stresses at the IOP of 10 and that of the $30 \mathrm{mmHg}$ for the retina and sclera, respectively. 
Moreover, it should be pointed out that the pressure inside the sclera in here would mostly be affected by the vitreous body which as it was shown that by a higher IOP it experienced a lower amount of von Mises stress. The highest stress in the retina was observed at the IOP of $30 \mathrm{mmHg}$ with $2.22 \mathrm{kPa}$ whereas the highest one in the sclera was occurred at the IOP of $10 \mathrm{mmHg}$ with $24.74 \mathrm{kPa}$. Similarly, the value of von Mises stress in the (a) ciliary body and (b) optic nerve was evaluated and displayed in Fig. 51.

The highest amount of stress for both the ciliary body and optic nerve was seen at the IOP of $30 \mathrm{mmHg}$ with the value of 44.31 and $11.5 \mathrm{kPa}$, respectively. Although this difference is considerable for the ciliary body compared to the other two IOPs, the results on the optic nerve showed almost the same values which are not much different. It shows that a higher amount of stress in the ciliary body would provide an unstable gripper/holder for the lens and affect the visual quality. In addition, it is observed that a higher amount of IOP would trigger a higher level of stress in the optic nerve and, consequently, makes it vulnerable to be damaged. To be more precise, the resultant displacement of the optic nerve due to IOP variation was computed and plotted in Fig. 52. Although a considerable resultant displacement has not been observed by the variation of IOP, the highest displacement was seen at the IOP of $30 \mathrm{mmHg}$ with $13.1 \mu \mathrm{m}$. Since no variation was observed in this regard, we decided to investigate the role of optic nerve/sclera elastic modulus on the $\mathrm{ONH}$ biomechanics in respect to the 
attachment site. To do that, two different elastic modulus for the optic nerve/sclera properties were selected.

\subsubsection{Optic Nerve Head (ONH) injury}

As it was pointed out the normal range of the IOP is $15 \mathrm{mmHg}$, however, in here the IOP was increased to figure out its correlation to the ONH biomechanics. Since the objective was to focus on the mechanical properties of the sclera/optic nerve, two different elastic modulus were compared, including the 1.65 (our experimental results) and 5.5 $\mathrm{MPa}$. The amount of deformation and stress in the $\mathrm{ONH}$ as a result of this increasing in the IOP were investigated and compared. The boundary of the eye model was as the exterior side of the extraconal fat as well as the posterior side of the intraconal fat were fixed. The results in the head of the optic nerve which is the critical site as a result of IOP increasing was investigated. The results in this regard were analyzed in terms of the deformation and stress in the attachment site of the ONH to the sclera and presented in Figs. 53 and 54 , respectively.

The nodes on the attachment site of the optic nerve to the sclera tissue were selected and the averaged values of the displacement in them were reported. The results in this regard showed that the deformation in the $\mathrm{ONH}$ depends on the mechanical properties of the sclera and the optic nerve in a way that the deformations of 10.42 and $16.02 \mu \mathrm{m}$ were observed at the $\mathrm{ONH}$ for the elastic modulus of the 5.50 and $1.65 \mathrm{MPa}$ for the sclera tissue. It should also be pointed 
out that the sclera and optic nerve had the same mechanical properties in this simulation.

Finally, the stresses in the interaction site of the sclera and retina at two different mechanical properties for the sclera, including the 1.65 (our data) and 5.50 (reference data) were calculated and their contours are presented in Fig. 55. The amount of stresses were including the 14.27 and $43.63 \mathrm{kPa}$ at the elastic moduli of 1.65 and $5.50 \mathrm{MPa}$, respectively. Since the amount of displacements in the head of the optic nerve were 16.02 and 10.42 at the elastic moduli of 1.65 and 5.50 MPa, respectively, therefore, it can be concluded that the elastic modulus of 5.50 $\mathrm{MPa}$ which brings about the stress $43.63 \mathrm{kPa}$ over-estimate the amount of stress in the interaction site of the sclera and retina.

\subsubsection{Discussions}

\subsubsection{IOP variation}

The cornea is the most anterior component of the eye which affects from the IOP of the aqueous body. By increasing the IOP, as it was expected, the amount of stress in the cornea is increased accordingly in a way that the highest stress was observed at the IOP of $30 \mathrm{mmHg}$ while the lowest one was observed at the IOP of $10 \mathrm{mmHg}$ (Fig. 46a). Although the direction of the applied load to the cornea expected to be in all area of the cornea surface, however, due to the structure/shape of the cornea the highest stress was located in the apex of the 
cornea. The stresses and deformations in the cornea showed a clear conical shape of the deformed cornea at the center of the cone where the cornea has the lowest thickness. This is in agreement with previous studies showed that in the advances condition of the keratoconus disease, a minor lessening of tissue thickness will trigger dramatic changes of the corneal form and, henceforth, the quality of vision [97]. Similarly, the stress in the lens had the same trend in a way that a higher amount of IOP invoked an inward load on the lens' surface and, as a result, a higher von Mises stress (Fig. 46b). Ophthalmologists believed that maximal corneal displacement specifically in the conic region of the cornea compared to its initial radius can be considered as a sign of the keratoconus severity [99]. Average value of the curvature of the anterior corneal surface is $7.84 \mathrm{~mm}$, while posterior radius is $6.4 \mathrm{~mm}$ [100]. These are in agreement with our results since our findings also well verified that IOP variation has no effect in the normal cornea with less than $8 \mathrm{~mm}$ variation [29]. The results proposed here simulated the various IOP values as $10-30 \mathrm{mmHg}$ in a way that the normal range is defined as IOP less than $20 \mathrm{mmHg}$ and the other values defined as the intense level of the disease. The morphological alterations of the cornea (Fig. 47a) and lens (Fig. 47b) were also verified the differences in the radius of curvature as a result of IOP increasing. That is, the highest curvature for both the cornea and lens was observed at the IOP of $30 \mathrm{mmHg}$ whereas he lowest one was observed at the IOP of $10 \mathrm{mmHg}$. In addition, the effects of the load direction in the inward and outward deformations of the lens and cornea is visible in their curves (Fig. 47). 
Since iris is the first component which is in direct interaction with the aqueous body in its posterior side, it is expected to have a higher deformation as a result of IOP variation. The results revealed the surface of the iris at a higher stress was profoundly affected by a higher IOP $(30 \mathrm{mmHg})$ while there is a less amount of stress distribution in the iris surface at the IOP of $10 \mathrm{mmHg}$ (Fig. 48). The vitreous body, regardless of the variations in the IOP, showed almost the same amount of stress with the same distribution (Fig. 49). The amount of stress in the retina and sclera which act as holder pressure applied from the external load was different by variation of the IOPs. That is, by increasing the pressure in the aqueous body the stress in the retina and sclera are increased and decreased, respectively (Fig. 50).

The crucial role of the optic nerve is to link the eye to the brain for image interpretation. In this study, the stresses and deformations of the optic nerve at various IOPs were also calculated (Figs. 51 and 52). The results illustrated that by increasing the IOP the stress in this component is amplified. The same pattern was observed in the resultant displacement of the optic nerve as a result of IOP increasing, since there was no dramatic alteration in the amount of displacement in the optic nerve head which can be lead to detachment from its site to sclera [101]. Increasing or decreasing of the IOP inside the eye can be accompanied by various type of injuries to the eye components such as over pressure/stress. Experimental measurement of these stresses in the eye components is not plausible and, there is a need to set up a numerical modeling to figure out what is 
the result of different amount of IOPs in the eye. Therefore, this study performed a numerical simulation to shed light on the role of IOP on the resultant stresses in the components of the eye.

Although it has been tried to propose a suitable model of the eye, there are still some simplifications that should be taken into account in future models. First, some of the human eye components have considered to behave like a linear-elastic isotropic material. In addition, some of them considered as isotropic hyperelastic or viscoelastic materials. However, strain energy density functions employing isotropic approach cannot input the role of collagen fiber directions into the material [102].

\subsection{2. $\mathrm{ONH}$ biomechanics}

A significant role of the IOP was observed on the ONH biomechanics [103] as some IOP-related injuries have been reported in the attachment site of the sclera and the optic nerve, namely $\mathrm{ONH}$ [7]. Some studies experimentally measured the displacement on the $\mathrm{ONH}$ as a result of the IOP alteration. Since the current study also performed at the same value of IOP, we can have a suitable comparison in this regard. The range of $18-32 \mu \mathrm{m}[104,105]$ was reported for the

ONH displacement at the IOP of $30 \mathrm{mmHg}$. The current study showed the value of $16.02 \mu \mathrm{m}$ which is almost close to this range compared to $10.42 \mu \mathrm{m}$. This well implies the importance of the sclera/optic nerve mechanical properties in the ONH biomechanics (Figs. 53 and 54). 
The results illustrated that by increasing the IOP the stress in this component is amplified regardless of the sclera/optic nerve mechanical properties. The same pattern was observed in the resultant displacement of the $\mathrm{ONH}$ as a result of IOP increasing. Although these values of displacement cannot lead to detachment of the optic nerve head from the sclera's surface, it can change the vision acuity and subsequent problems [101].

Finally, the stresses in the interaction site of the sclera and retina at two different mechanical properties for the sclera, including the 1.65 (our data) and 5.50 (reference data) were calculated (Fig. 55). The amount of stresses were including the 14.27 and $43.63 \mathrm{kPa}$ at the elastic moduli of 1.65 and $5.50 \mathrm{MPa}$, respectively. Since the amount of displacements in the head of the optic nerve were 16.02 and 10.42 at the elastic moduli of 1.65 and $5.50 \mathrm{MPa}$, respectively, therefore, we can say the elastic modulus of 5.50 MPa which lead to the stress of $43.63 \mathrm{kPa}$ may over-estimate the amount of stress in the interaction site of the sclera and retina. 
Table 10. The amount of stress in the eye components at two different material model for the iris, including the elastic and hyperleastic ones.

\begin{tabular}{lcc}
\hline Components & Elastic Iris & Hyperelastic Iris \\
\hline Muscle & $2.25 \mathrm{kPa}$ & $2.13 \mathrm{kPa}$ \\
Intraconal fat & $106.8 \mathrm{~Pa}$ & $110 \mathrm{~Pa}$ \\
Cornea & $8.07 \mathrm{MPa}$ & $8.10 \mathrm{MPa}$ \\
Sclera & $32.51 \mathrm{kPa}$ & $32.9 \mathrm{kPa}$ \\
Retina & $7.58 \mathrm{kPa}$ & $7.60 \mathrm{kPa}$ \\
Vitreous body & $2.14 \mathrm{~Pa}$ & $1.77 \mathrm{~Pa}$ \\
Aqueous body & $260.7 \mathrm{~Pa}$ & $271.2 \mathrm{~Pa}$ \\
Ciliary body & $14.96 \mathrm{kPa}$ & $15.26 \mathrm{kPa}$ \\
Lens & $10.70 \mathrm{kPa}$ & $11.10 \mathrm{kPa}$ \\
Iris & $2.28 \mathrm{kPa}$ & $19.20 \mathrm{MPa}$ \\
Optic nerve & $236.2 \mathrm{~Pa}$ & $230.3 \mathrm{~Pa}$ \\
Extraconal fat & $15.05 \mathrm{~Pa}$ & $14 \mathrm{~Pa}$ \\
\hline
\end{tabular}


Table 11. The amount of stress in the eye components at two different material model for the iris, including the elastic and hyperleastic ones. The results in here are reported at the IOP value of $30 \mathrm{mmHg}$.

\begin{tabular}{lcc}
\hline Components & Elastic Iris & Hyperelastic Iris \\
\hline Muscle & $11.81 \mathrm{kPa}$ & $11.81 \mathrm{kPa}$ \\
Intraconal fat & $230.5 \mathrm{~Pa}$ & $230.5 \mathrm{~Pa}$ \\
Cornea & $244.5 \mathrm{kPa}$ & $244.5 \mathrm{kPa}$ \\
Sclera & $23.04 \mathrm{kPa}$ & $23.05 \mathrm{kPa}$ \\
Retina & $2.16 \mathrm{kPa}$ & $2.16 \mathrm{kPa}$ \\
Vitreous body & $2.69 \mathrm{~Pa}$ & $2.69 \mathrm{~Pa}$ \\
Aqueous body & $1.51 \mathrm{~Pa}$ & $1.55 \mathrm{~Pa}$ \\
Ciliary body & $44.44 \mathrm{kPa}$ & $44.23 \mathrm{kPa}$ \\
Lens & $19.29 \mathrm{kPa}$ & $19.38 \mathrm{kPa}$ \\
Iris & $10.25 \mathrm{kPa}$ & $26.51 \mathrm{kPa}$ \\
Optic nerve & $9.63 \mathrm{kPa}$ & $11.50 \mathrm{kPa}$ \\
Extraconal fat & $74.07 \mathrm{~Pa}$ & $74.97 \mathrm{~Pa}$ \\
\hline
\end{tabular}




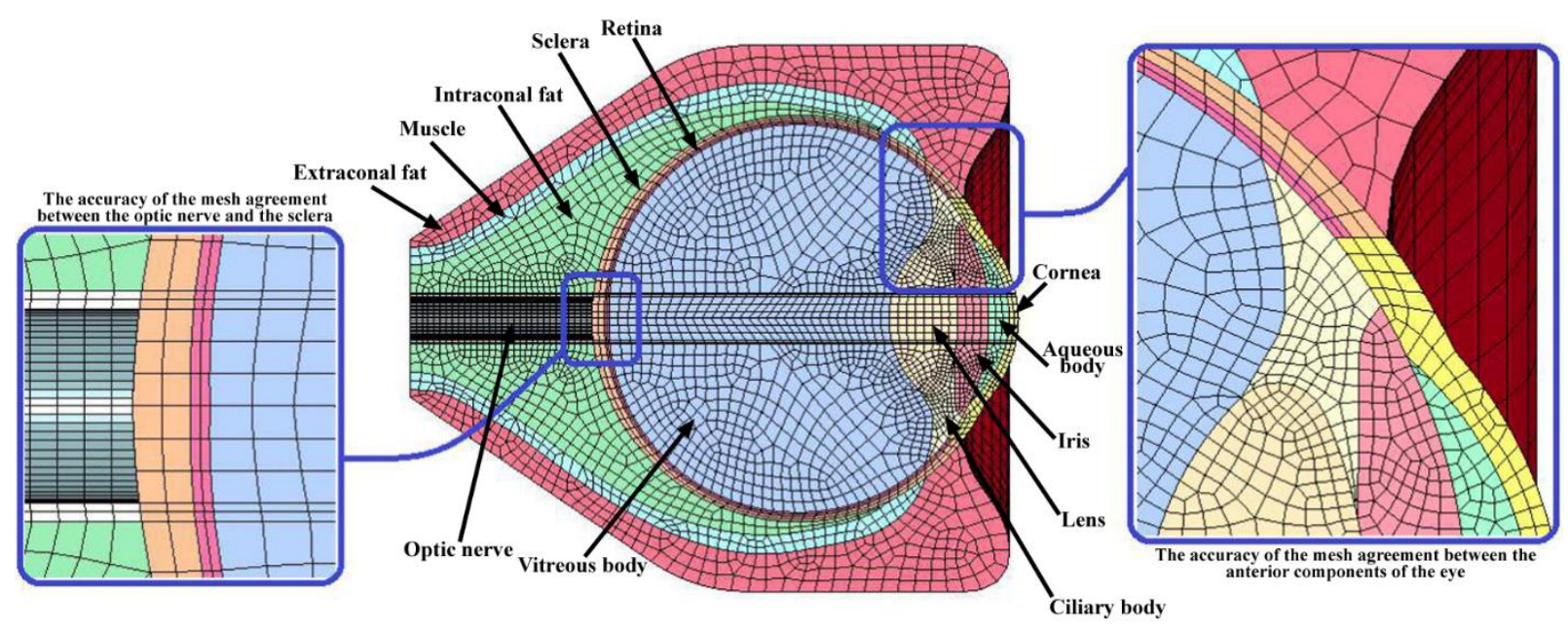

Fig. 35. The meshed finite element model of the human eye, including the extraconal fat, intraconal fat, aqueous, cornea, iris, vitreous body, retina, sclera, muscle, ciliary body, lens, macula, and optic nerve. The eye model was established according to the anatomical data of the real human eye. In addition, due to the complexity of meshing as a result of an intricate structure of the eye components, the accuracy of the node to node coupling in both the anterior and posterior components of the eye are presented. 

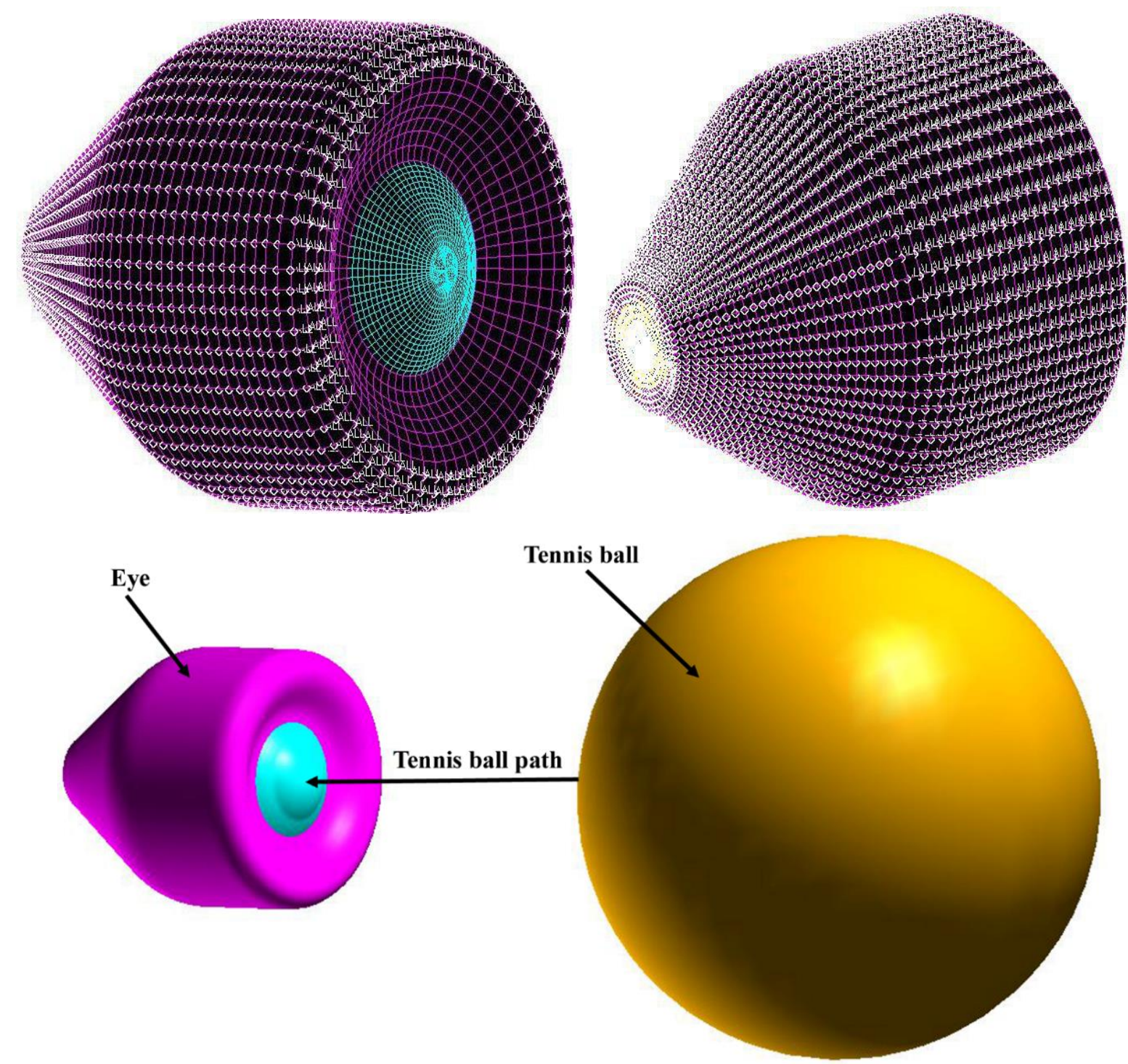

Fig. 36. The boundary condition of the from the front and back views. The extra side of the extraconal fat was all fixed since in reality also it is located inisde of the eye bone. The tennis ball was directly hit the conrea. 


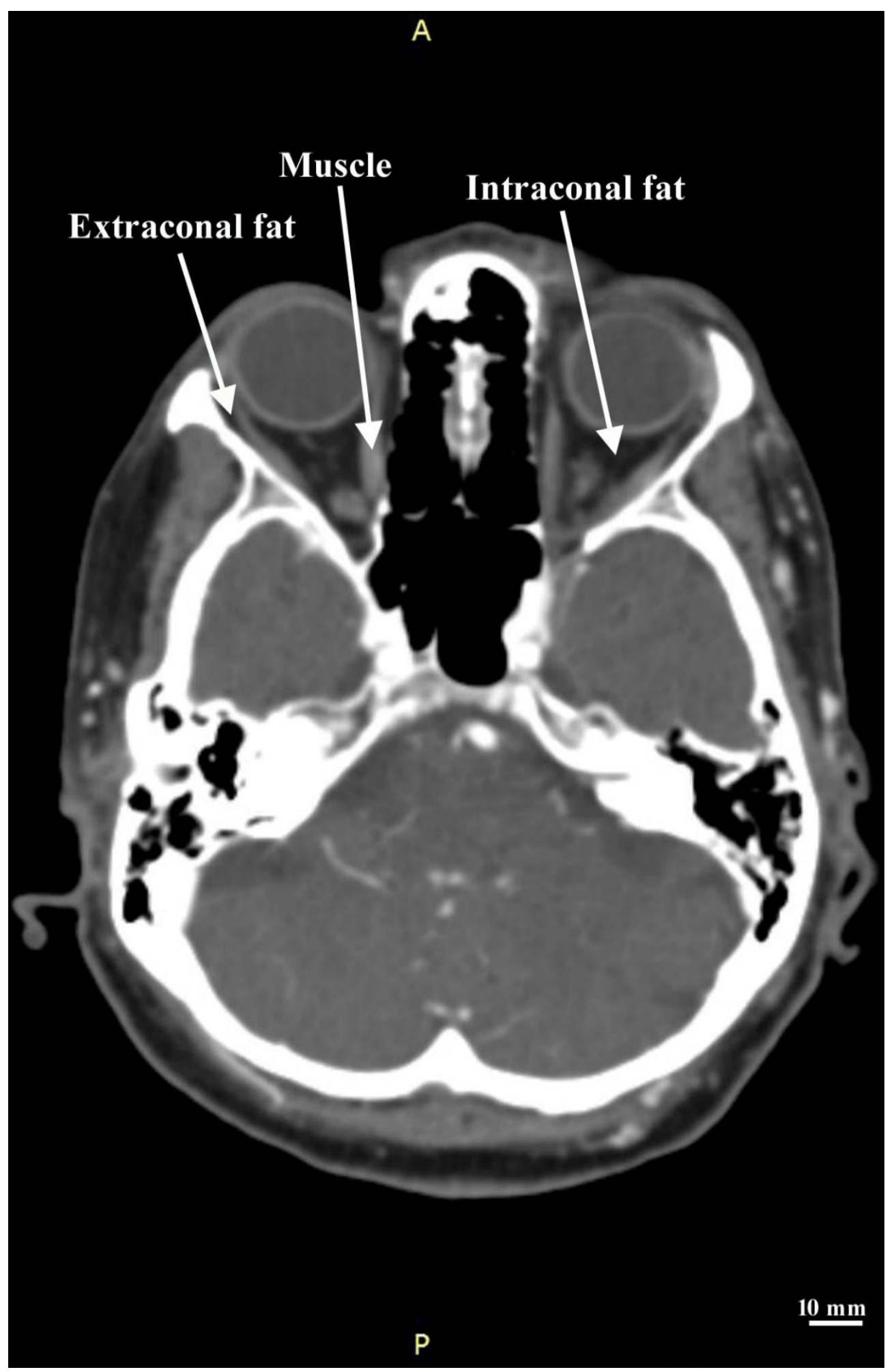

Fig. 37. The CT data of the intra and extraconal fats, and muscle. 


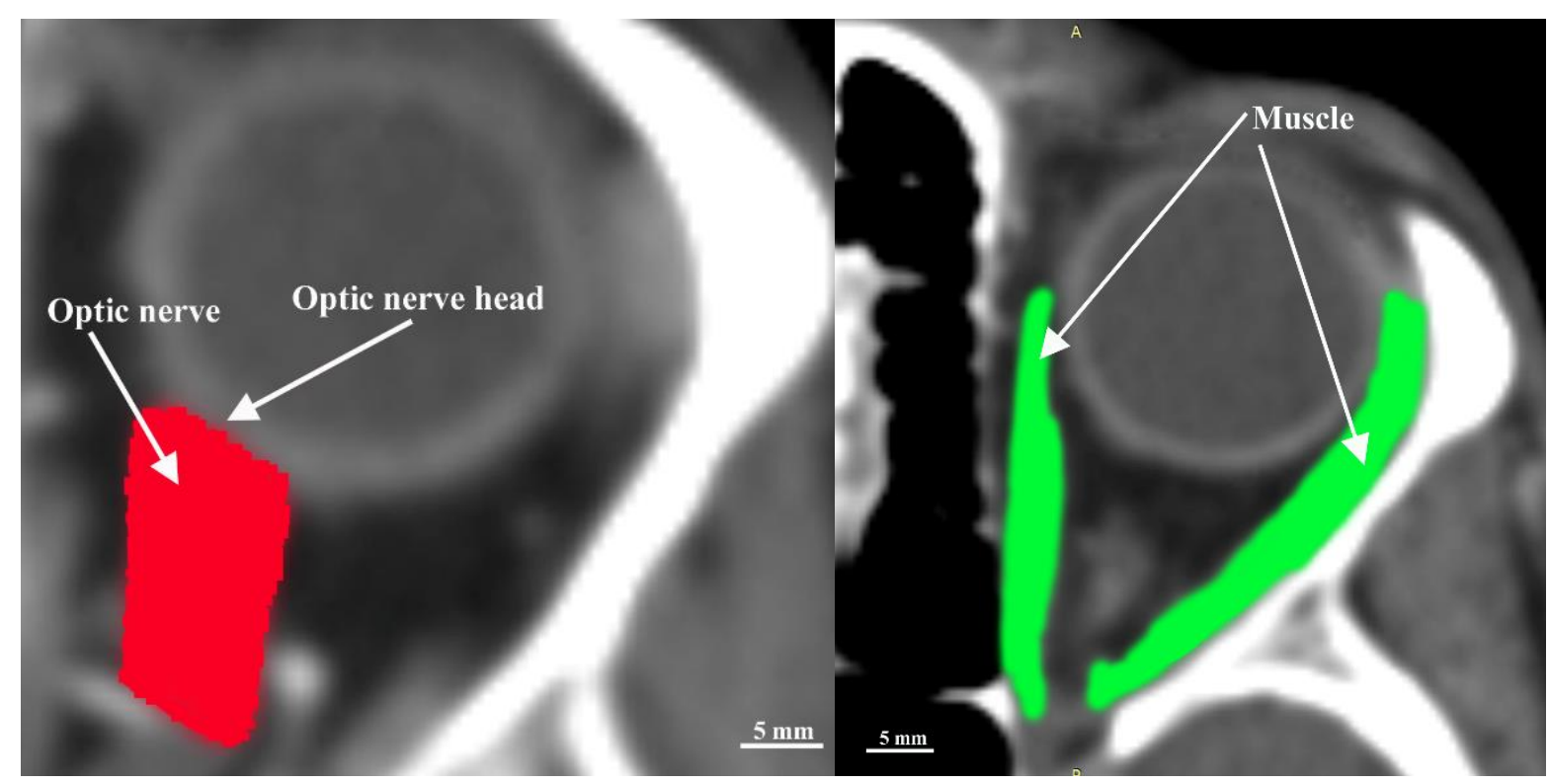

Fig. 38. The CT data of the optic nerve/optic nerve head and muscle. 


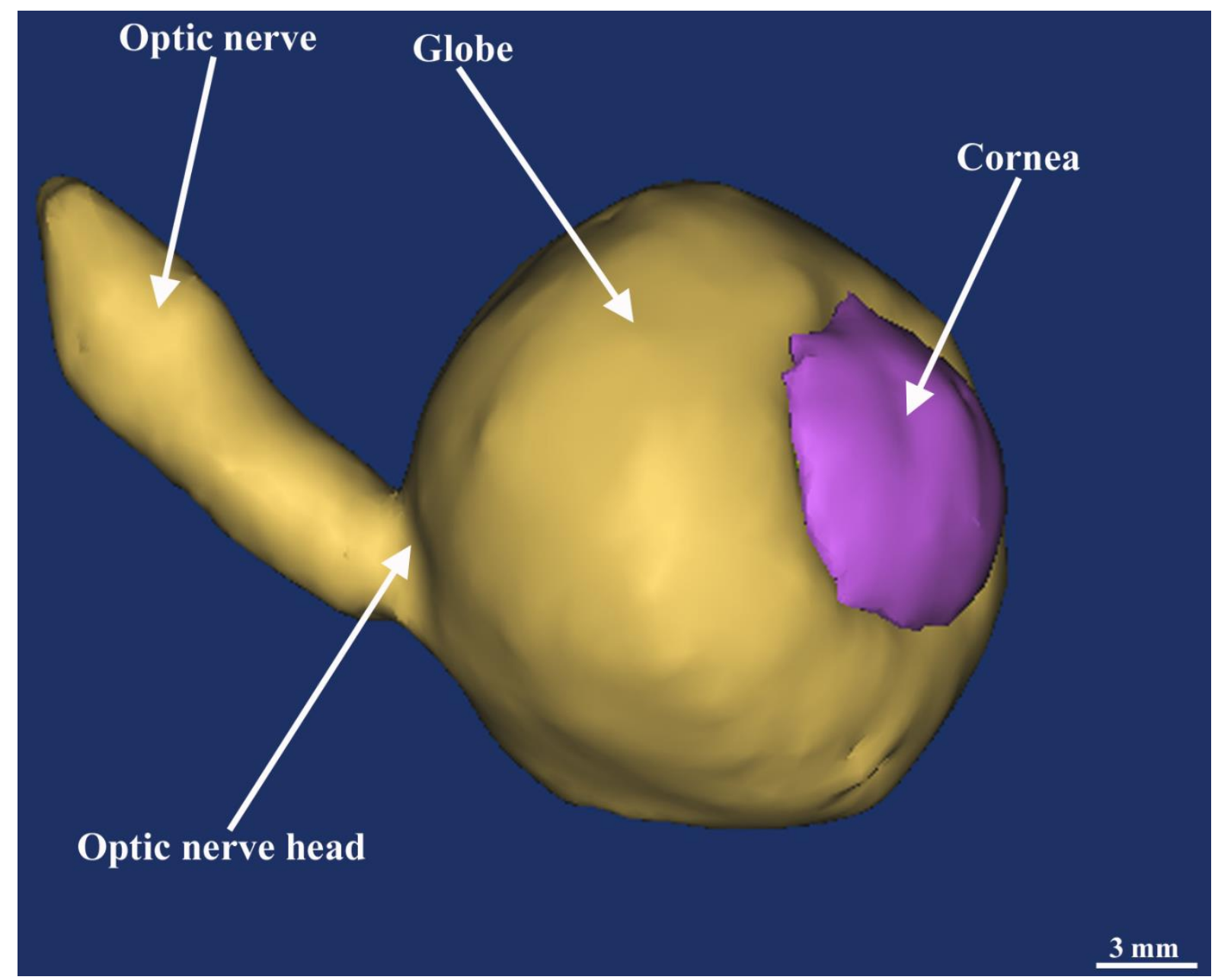

Fig. 39. The 3D model of the eye outputted from the Mimics software with a focus on the optic nerve head and the optic nerve. 


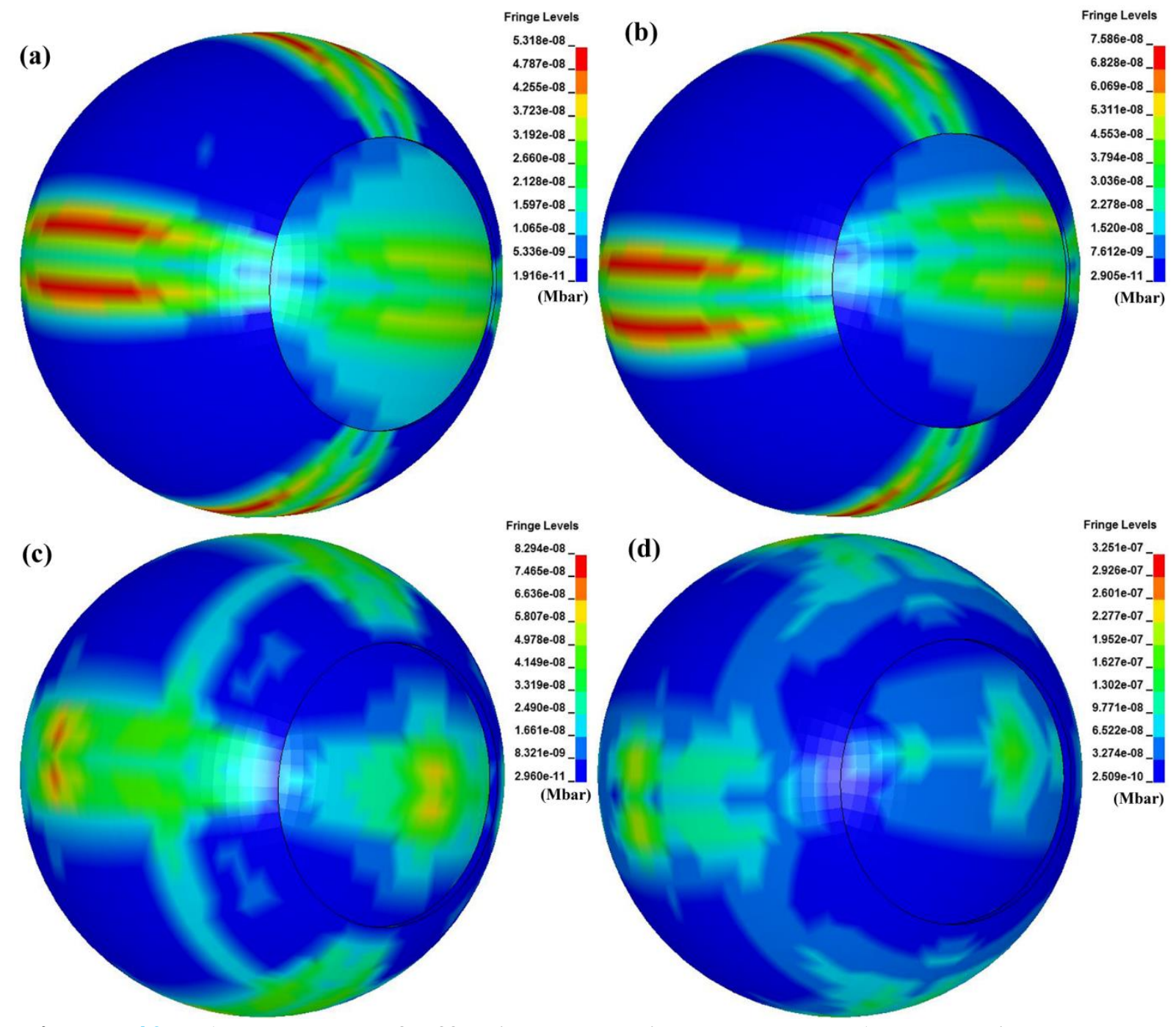

Figure 40. The contour of effective/von Mises stress on the (a) retina $(E=1.65$ $\mathrm{MPa})$, (b) retina $(\mathrm{E}=5.50 \mathrm{MPa})$, (c) sclera (1.65 MPa), and (d) sclera $(\mathrm{E}=5.50$ $\mathrm{MPa})$. These two elastic modulus are related to our data $(\mathrm{E}=1.65 \mathrm{MPa})$ and the reference data $(\mathrm{E}=5.50 \mathrm{MPa})$. 


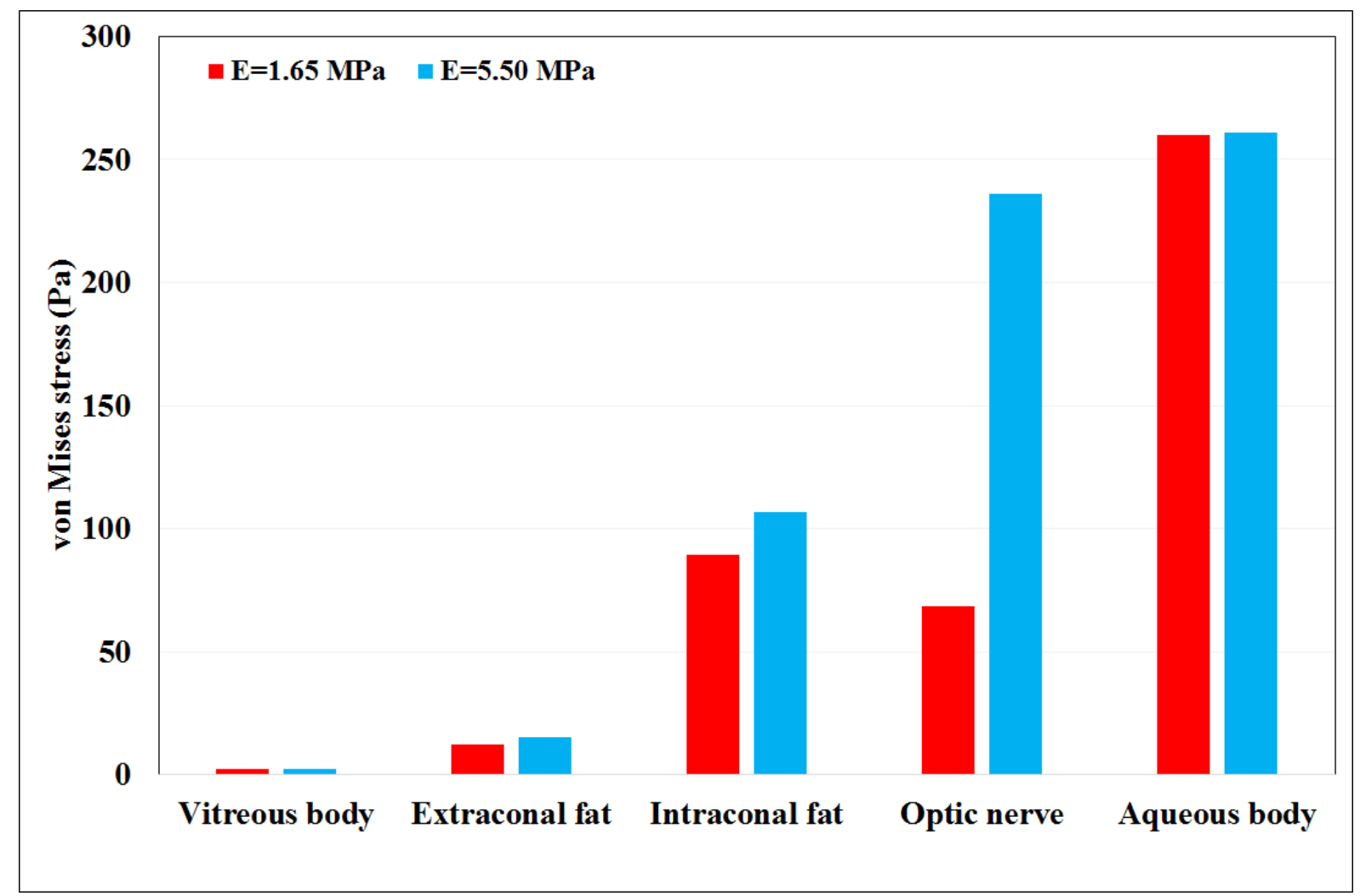

Figure 41. A comparative histogram representation of the von Mises stresses on the eye components, including vitreous body, extraconal and intraconal fat, optic nerve, and aqueous body. 


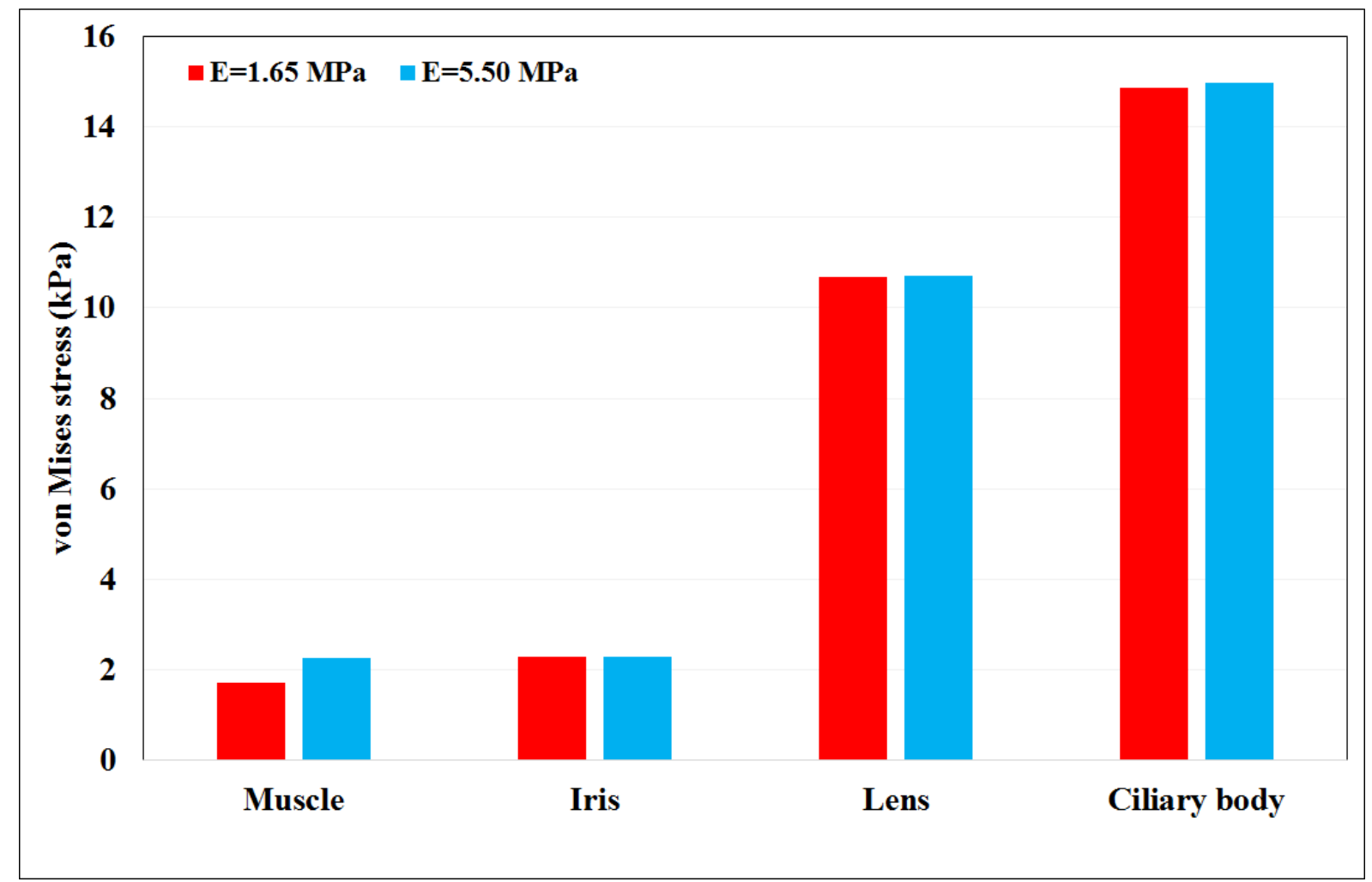

Figure 42. A comparative histogram representation of the von Mises stresses on the eye components, including muscle, iris, lens, and ciliary body. 

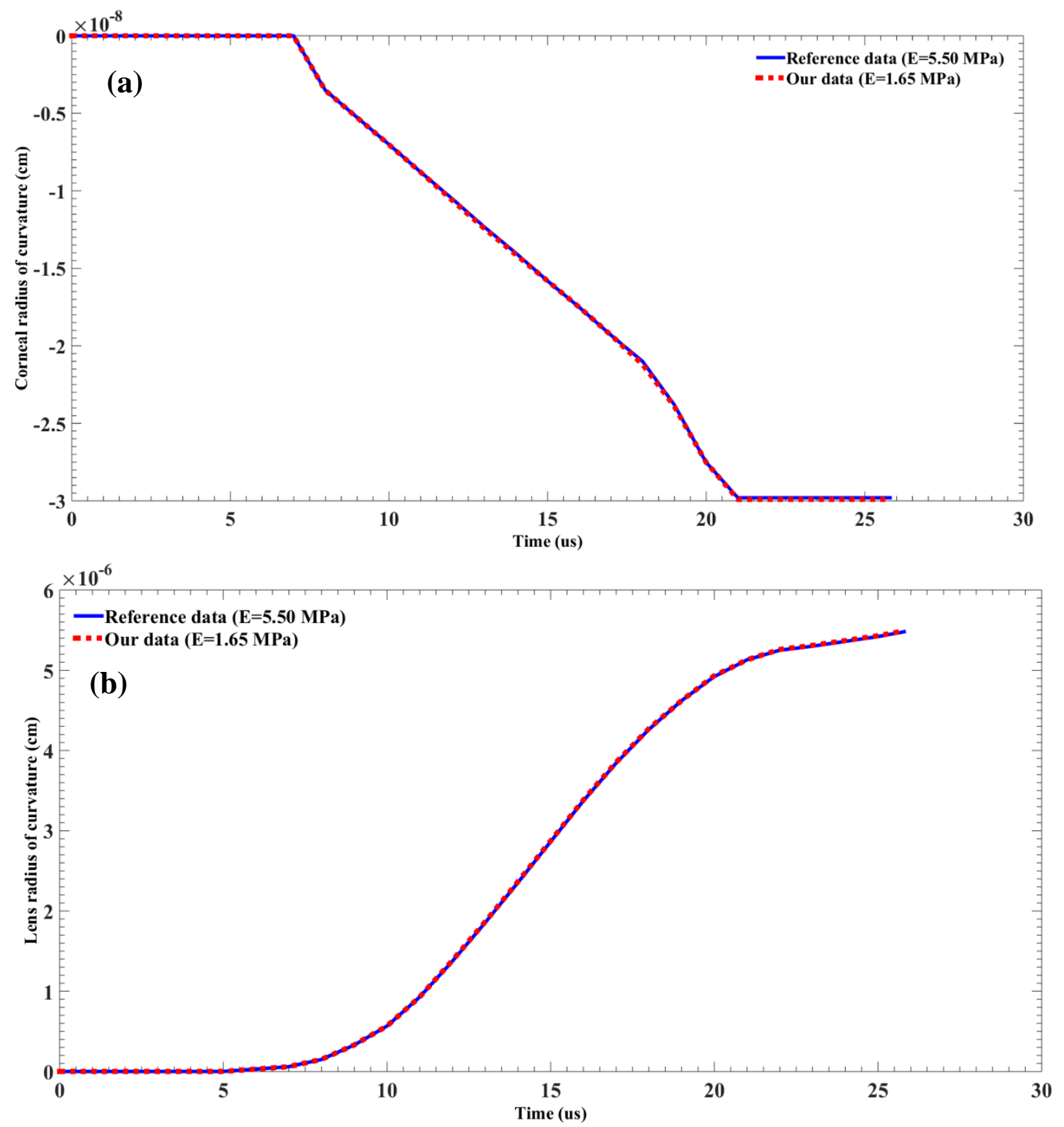

Figure 43. Diagrams of the radius of curvatures versus the simulation time for the (a) cornea and (b) lens. 


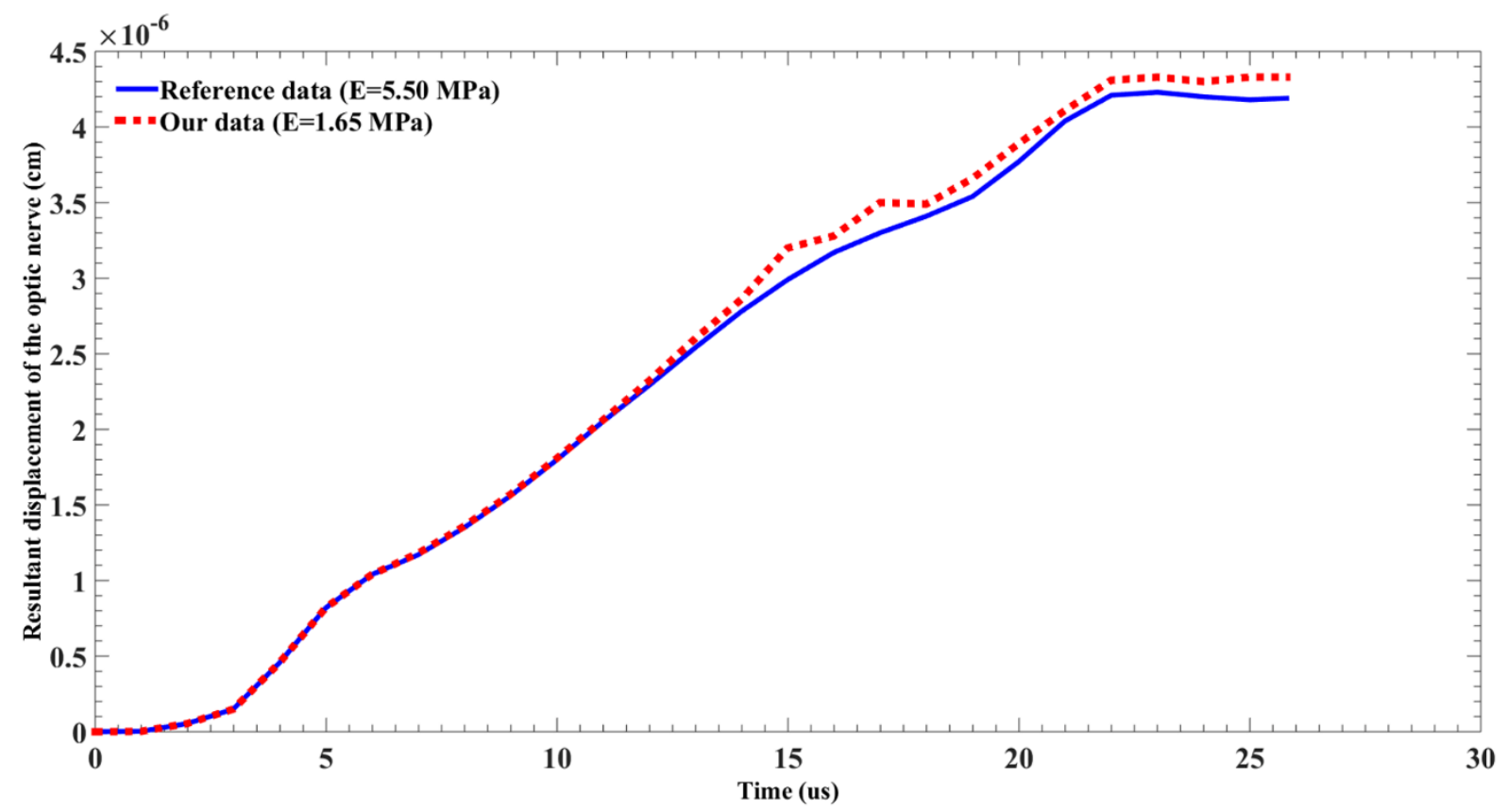

Figure 44. Diagrams of the resultant displacement versus the simulation time for the optic nerve. 


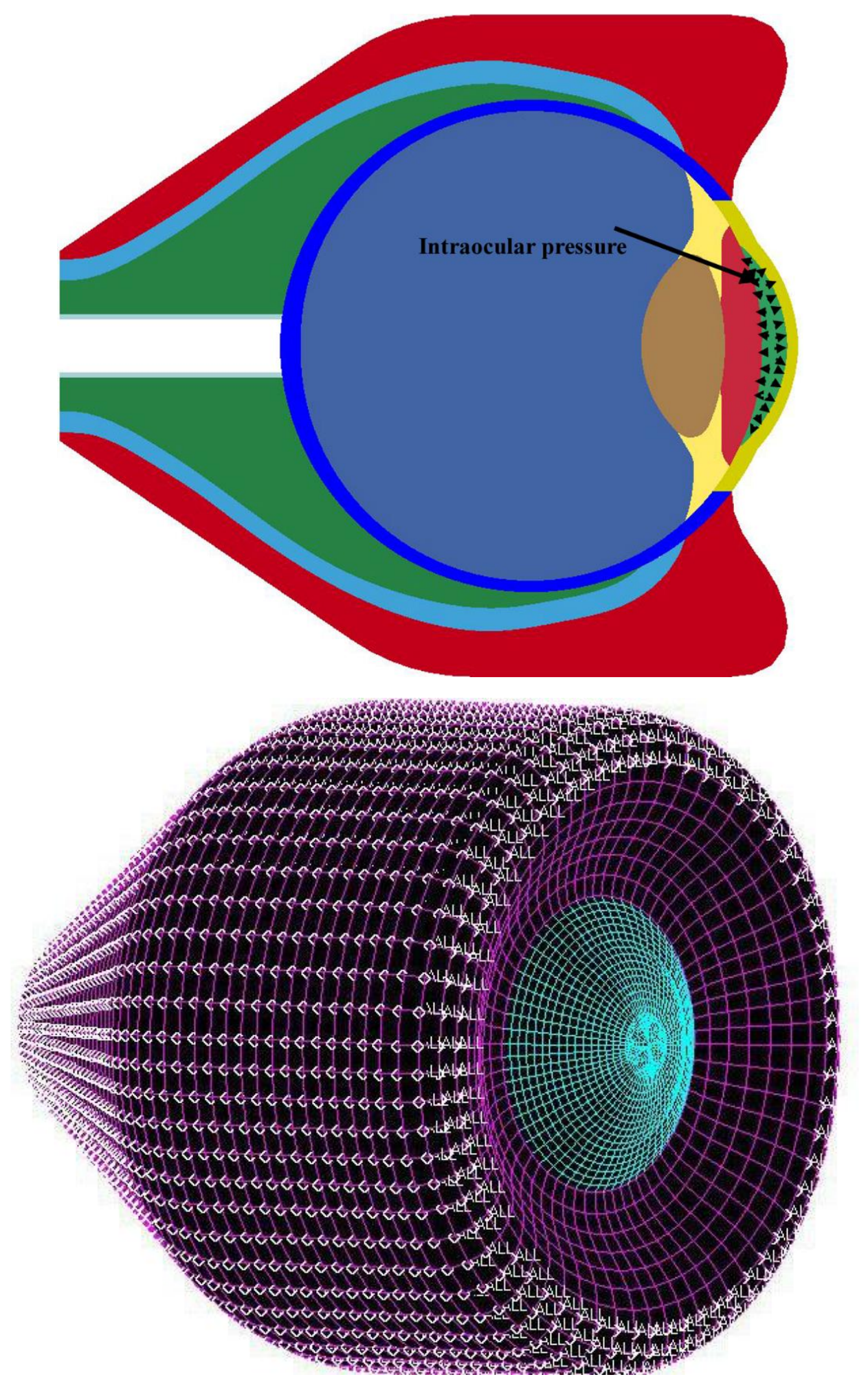

Fig. 45. The boundary condition of the from the front and back views. The extra side of the extraconal fat was all fixed since in reality it is located inisde of the eye bone. The IOP was applied to the components which are in direct contact with the aqueous body. 


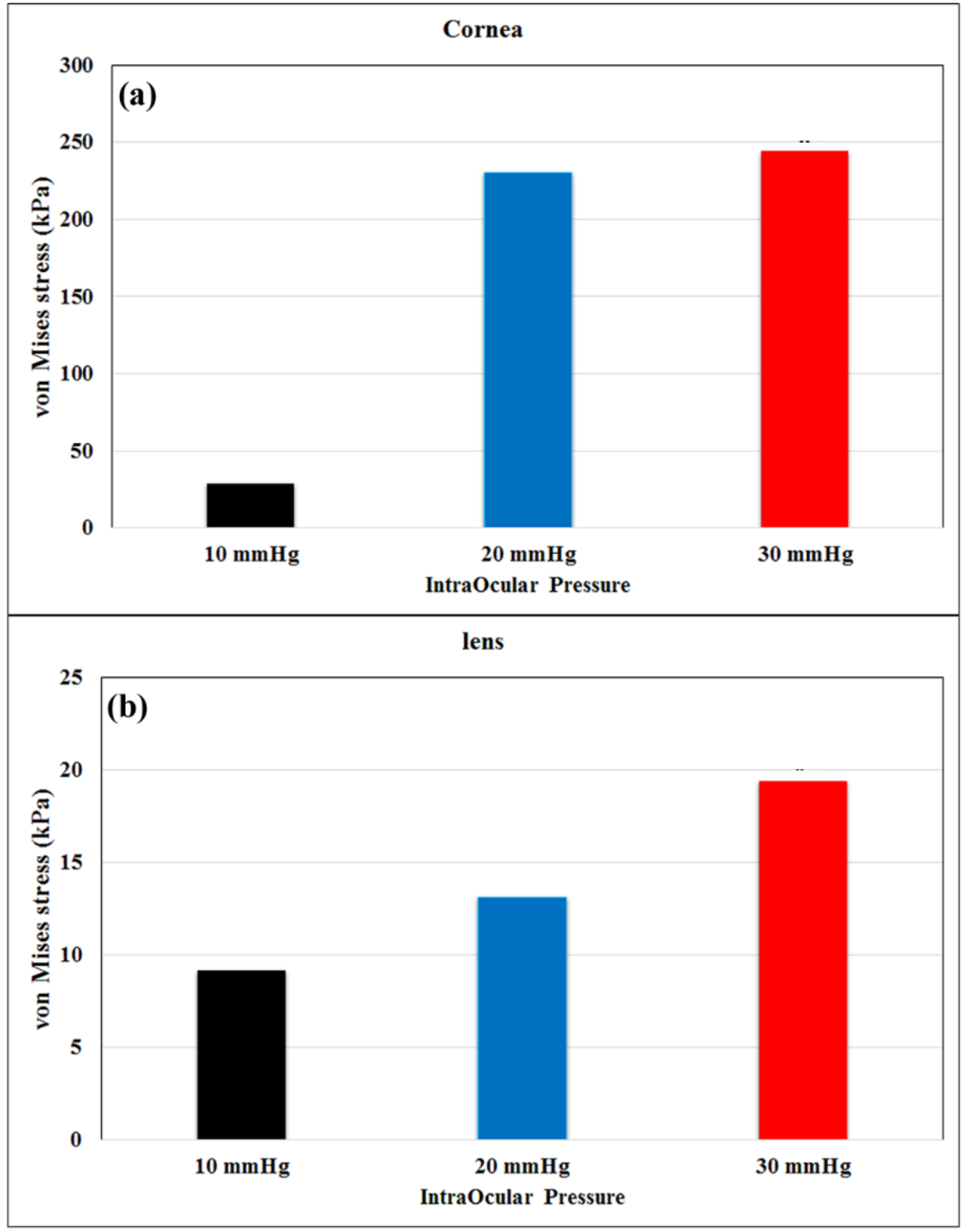

Fig. 46. A comparative bar chart represanation of the von Mises stresses on (a) the cornea and (b) the lens at different Intraocular Pressures (IOPs), such as 10, 20, and $30 \mathrm{mmHg}$. 

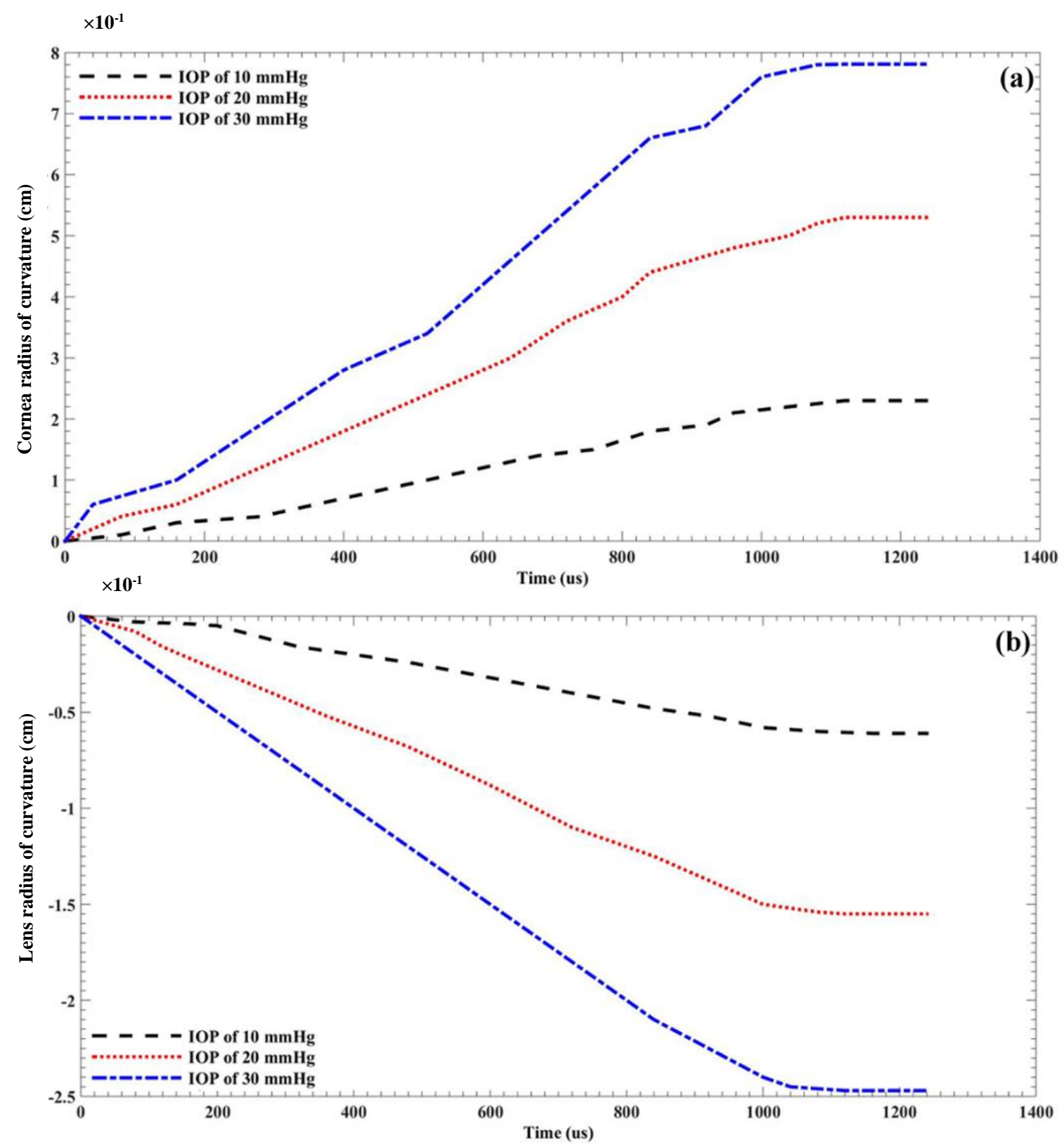

Fig. 47. A comparative diagram of the radius of curvature at three different Intraocular Pressures (IOPs) for (a) the cornea and (b) the lens. 


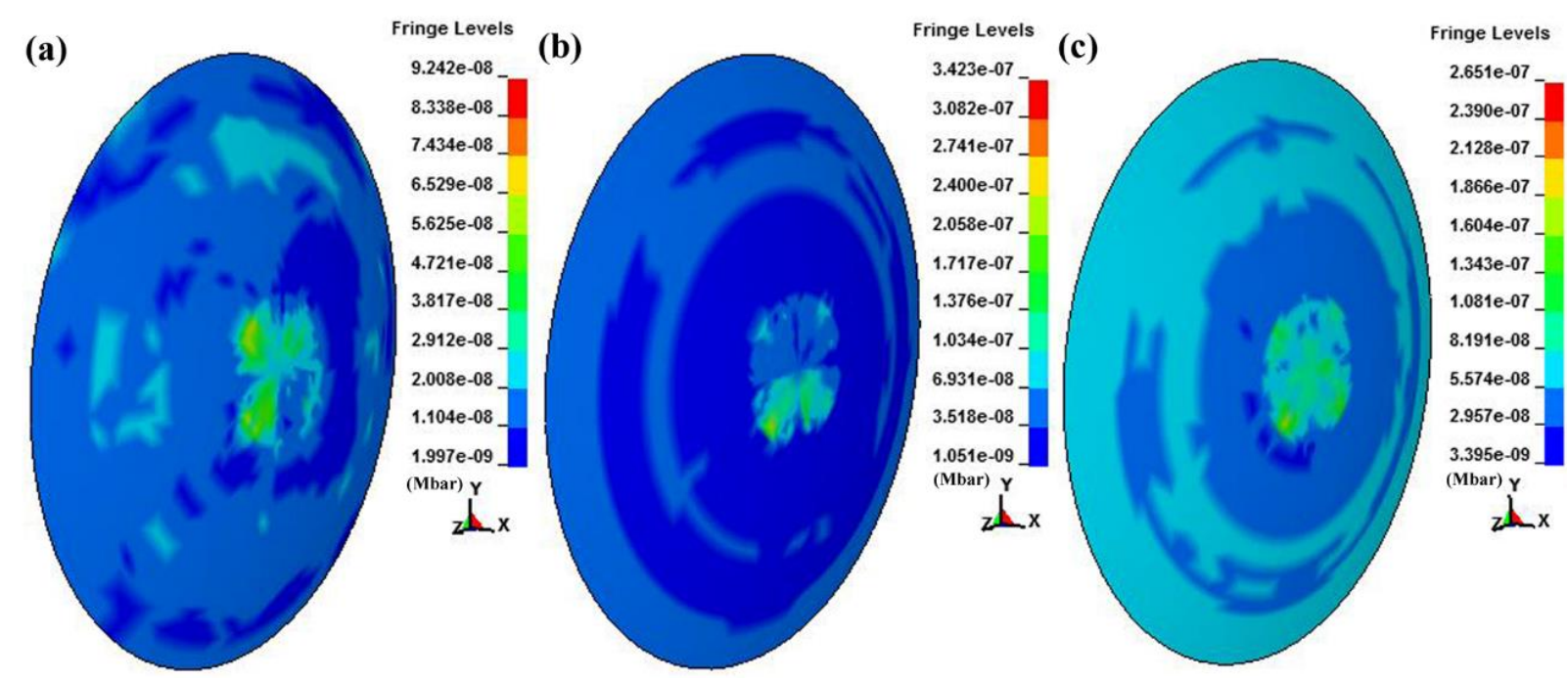

Fig. 48. The contour of von Mises stress on the iris at three different Intraocular

Pressures (IOPs), i.e., (a) 10, (b) 20, and (c) $30 \mathrm{mmHg}$. 

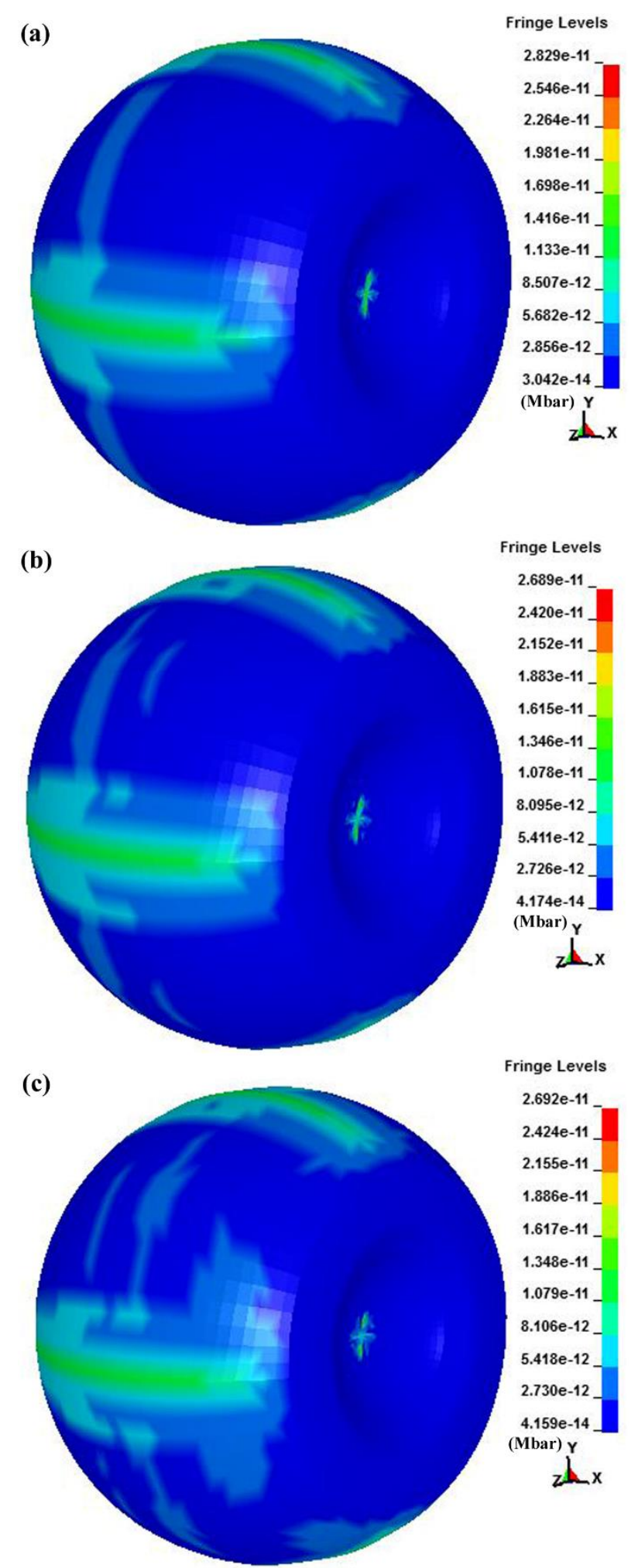

Fig. 49. The contour of von Mises stress on the vitreous body at three different Intraocular Pressures (IOPs), i.e., (a) 10, (b) 20, and (c) $30 \mathrm{mmHg}$. 


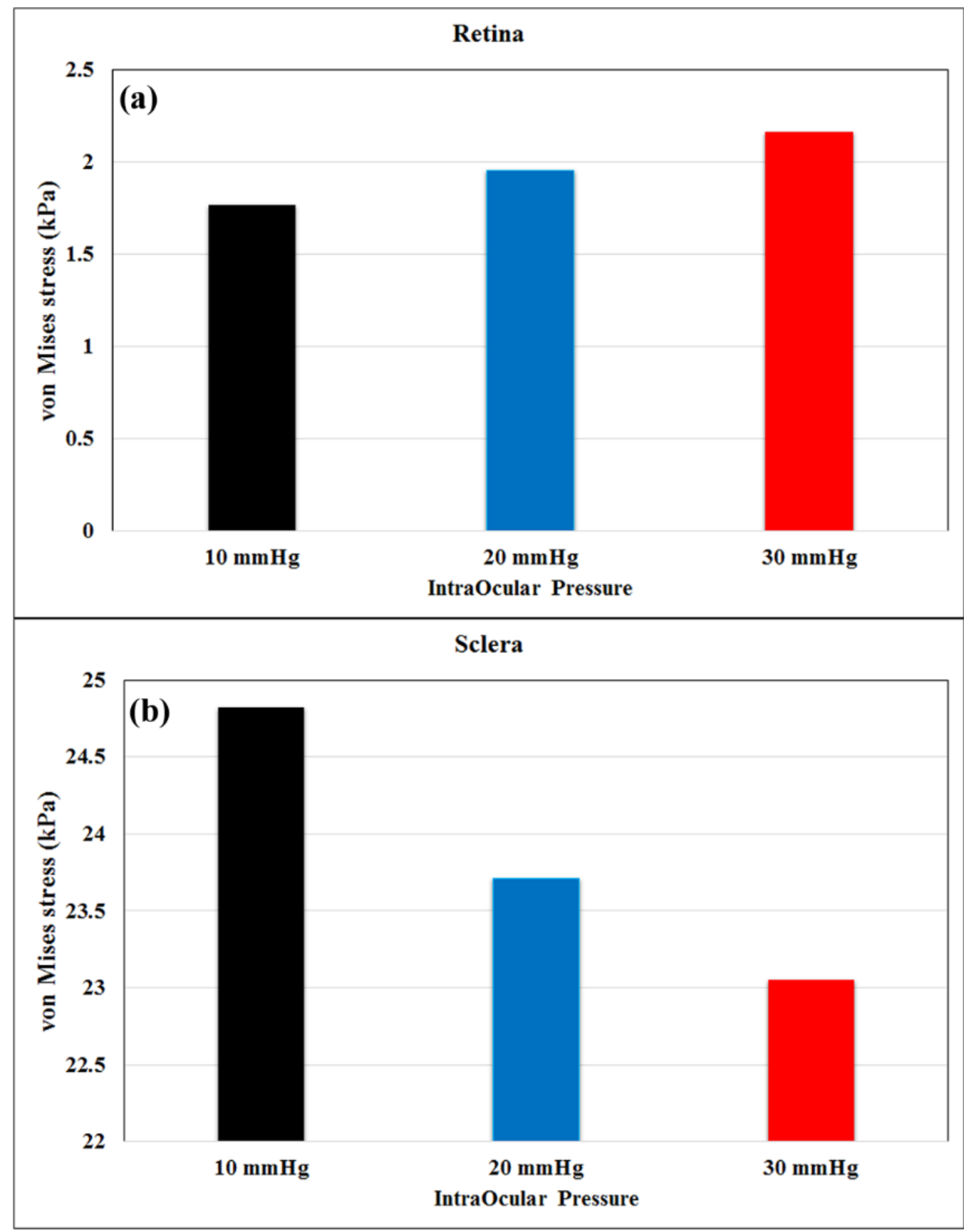

Fig. 50. A comparative bar chart represanation of the von Mises stresses on (a) the retina and (b) the sclera at different Intraocular Pressures (IOPs), such as 10, 20, and $30 \mathrm{mmHg}$. 


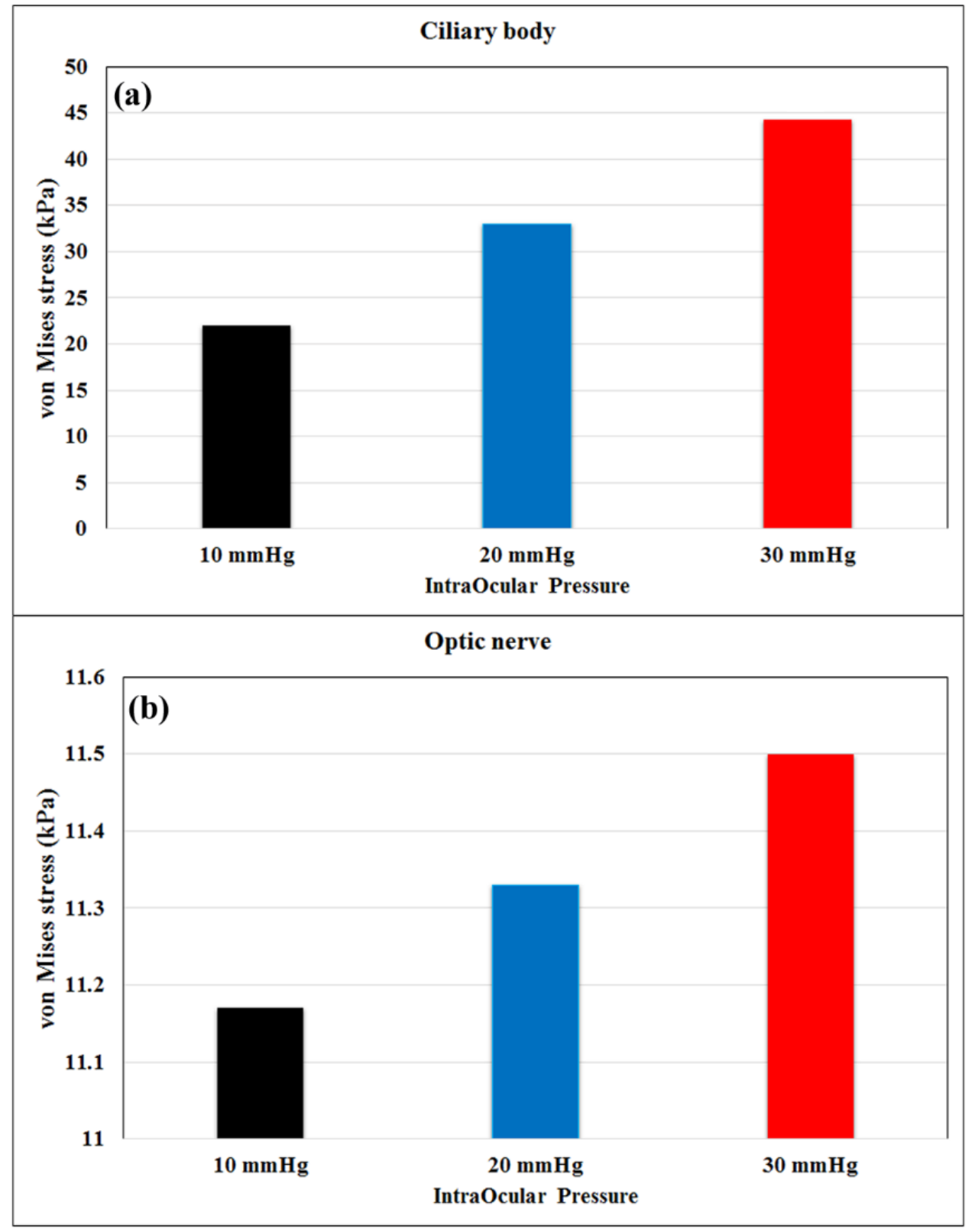

Fig. 51. A comparative bar chart represanation of the von Mises stresses on (a) the ciliary body and (b) the optic nerve at different Intraocular Pressures (IOPs), such as 10,20 , and $30 \mathrm{mmHg}$. 


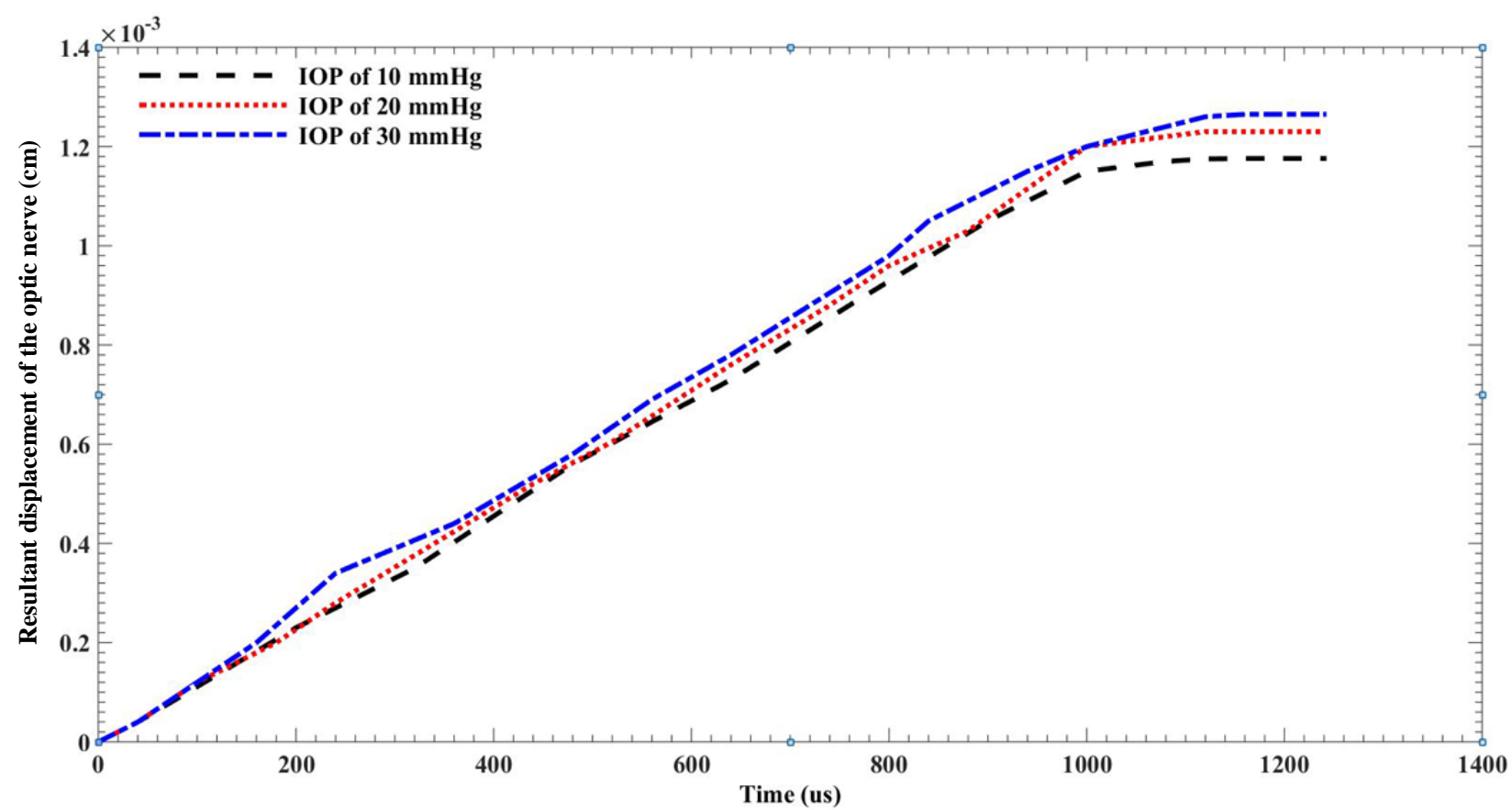

Fig. 52. A comparative diagram of the resultant displacement at three different Intraocular Pressures (IOPs) for the optic nerve. 


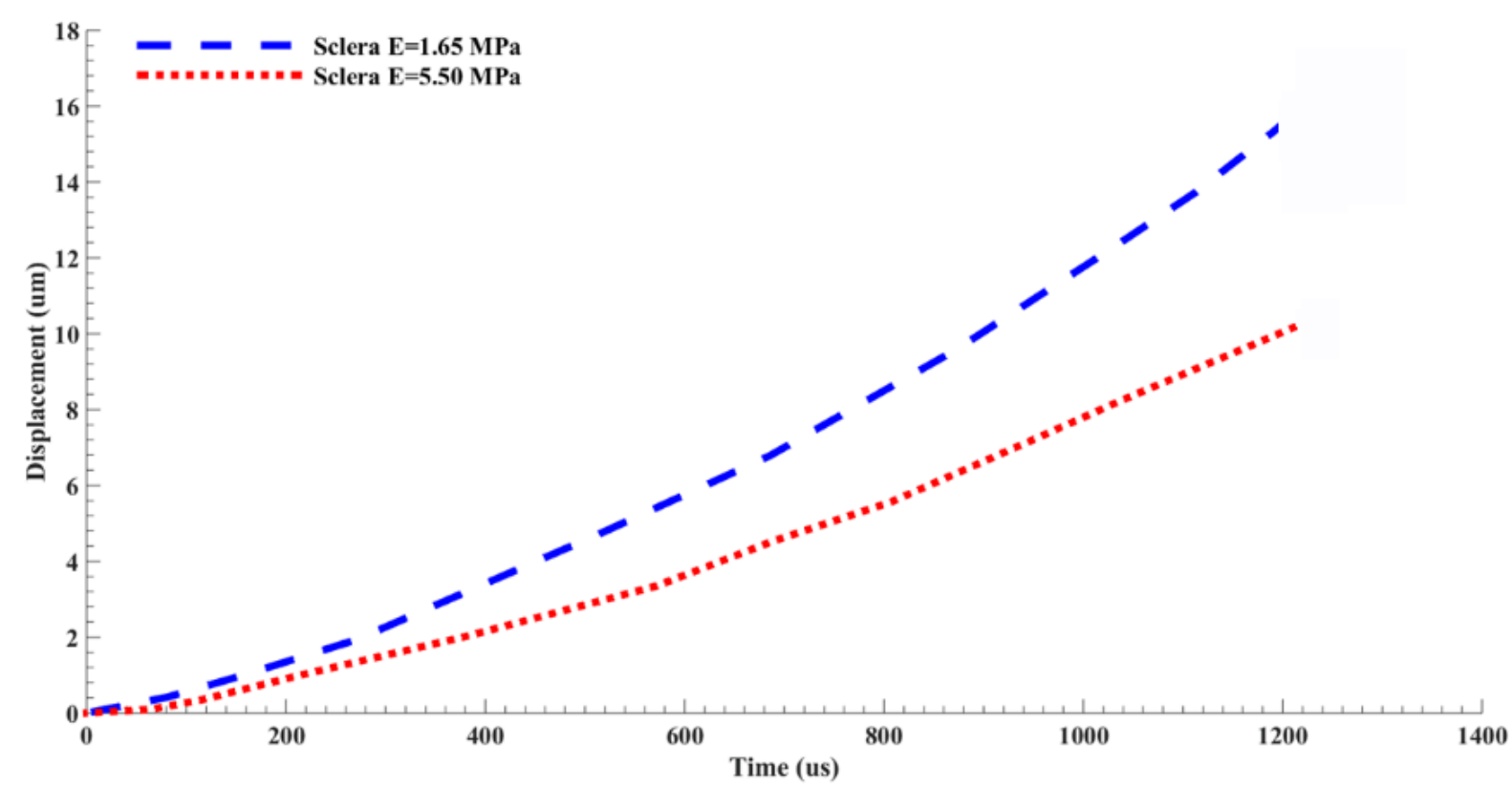

Fig. 53. In order to emphasize on the importance of the sclera/optic nerve mechanical properties, two different elastic modulus, including $\mathrm{E}=1.65 \mathrm{MPa}$ and $\mathrm{E}=5.50 \mathrm{MPa}$, were assigned to the model and simulated. In here, the displacement on the $\mathrm{ONH}$ as a result of these two elastic modulus are plotted. 


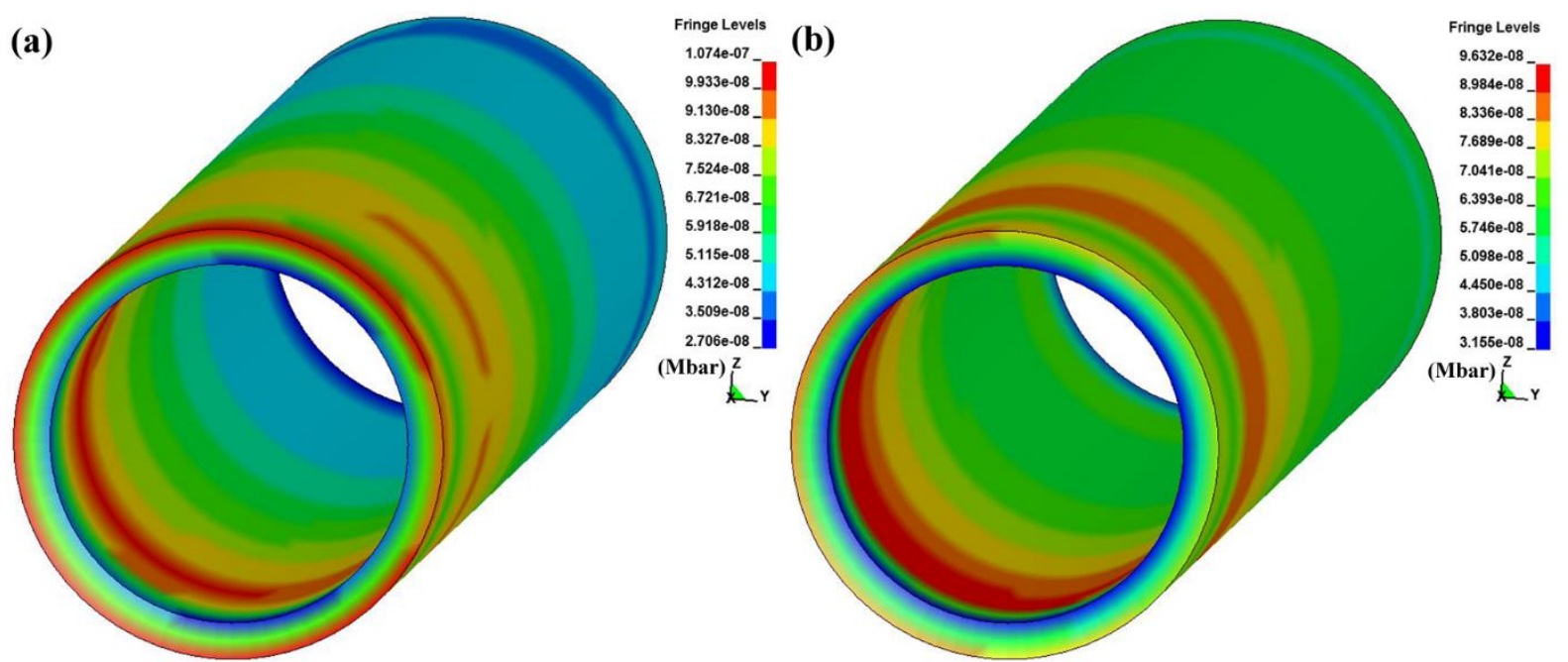

Fig. 54. In order to emphasize on the importance of the sclera/optic nerve mechanical properties, two different elastic modulus, including (a) E=1.65 $\mathrm{MPa}$ and (b) $\mathrm{E}=5.50 \mathrm{MPa}$, were assigned to the model and simulated. In here, the von Mises stress contour as a result of these two elastic modulus are displayed. 


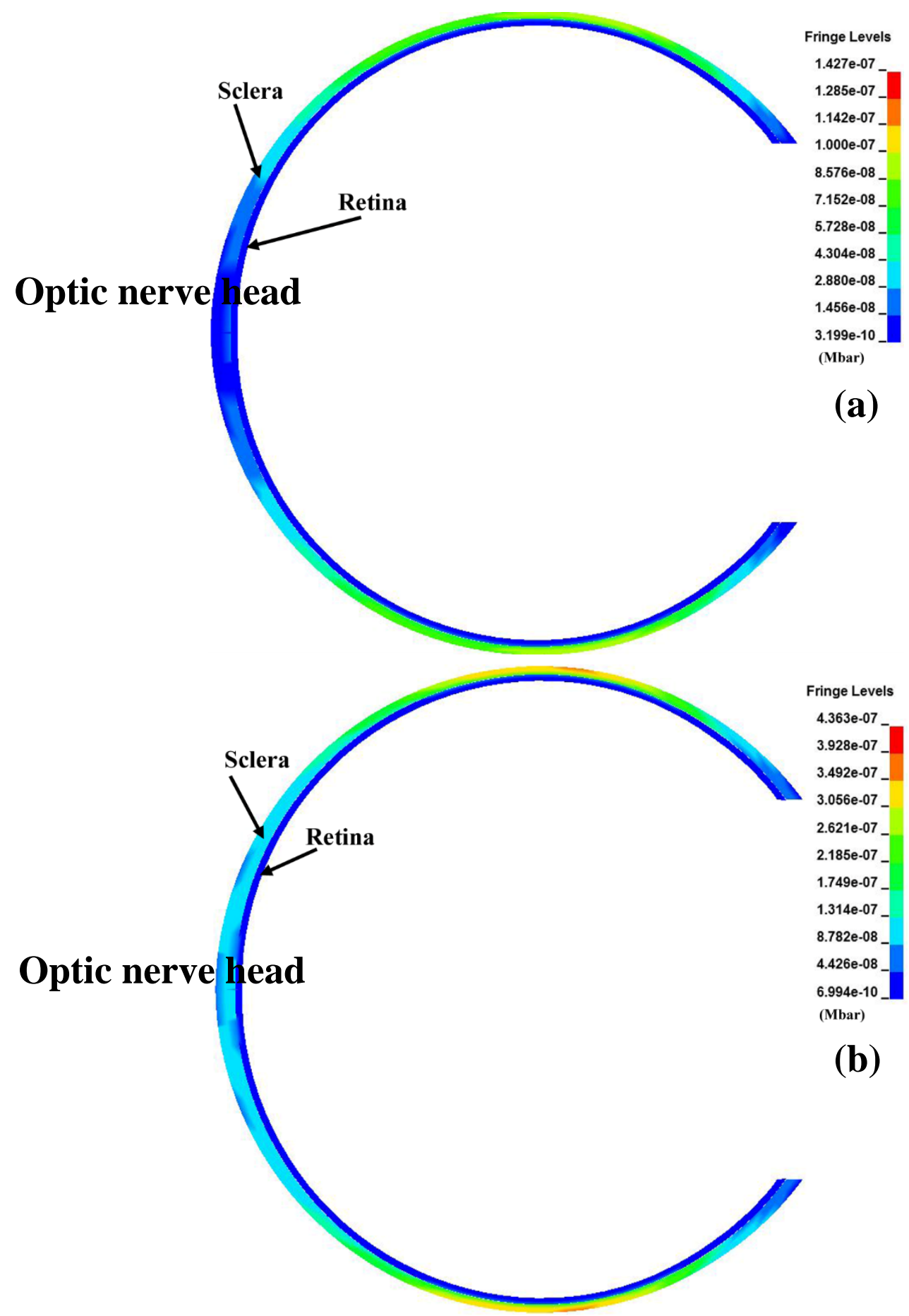

Fig. 55. The contour of stress between the sclera and retina at two different elastic modulus, including (a) $\mathrm{E}=1.65 \mathrm{MPa}$ (our data) and (b) $\mathrm{E}=5.50 \mathrm{MPa}$ (Reference data). 


\section{Chapter 5 \\ Conclusions}


In this section which is the last section of this thesis, the conclusions of this thesis point by point are briefly described as following.

The objective of the current study was to propose a suitable anatomical-based computational FSI model according to the experimental data to be used for eye injury simulations. Since a suitable eye model should be enriched with a suitable mechanical properties as well as experimental validation, these were fulfilled thru the experimental analyses. To do that, the mechanical properties of the sclera were experimentally measured thru the linear elastic, nonlinear hyperelastic, and linear viscoelastic models under different strain rates. The sclera showed the least elastic modulus at the strain rate of $5 \mathrm{~mm} / \mathrm{min}$ with $1.10 \mathrm{MPa}$ while the highest one was observed at the strain rate of $200 \mathrm{~mm} / \mathrm{min}$ with $2.92 \mathrm{MPa}$. The MooneyRivlin, Ogden, and Yeoh hyperelastic material models showed suitable agreement with the experimental data and therefore can be employed to address the nonlinear mechanical behavior of the sclera tissue. Meanwhile, the CT/MRI data along with the data reported in the literature were employed to establish an anatomical-based eye model. For the certification of the model, its accuracy was experimentally and numerically verified using the penetrating test under five different strain rates. Our anatomical-based FSI model was also numerically compared to the stress-displacement data of other three common models in the field, and the pros of our model in terms of the mechanical response was well observed. The models were consisted of the linearly simple elastic model as the $1^{\text {st }}$ model. The $2^{\text {nd }}$ model was made of the cornea, sclera, lens, and the optic nerve. 
The $3^{\text {rd }}$ model or VTEM-based model made of the cornea, aqueous body, ciliary body, zonules, sclera, lens, and vitreous body while the roles of the iris and retina in their model were ignored. Although our anatomical-based eye model was established on a basis of the $3^{\text {rd }}$ model, since they have not taken the role of the iris into account, at an specific displacement in the apex of the cornea, the $3^{\text {rd }}$ model showed a lower value compared to our anatomical-based model. For example, at $1 \mathrm{~mm}$ deformation in the apex of the cornea, the experimental data showed the value of $50 \mathrm{kPa}$ while the $3^{\text {rd }}$ model displays the value of $17 \mathrm{kPa}$ and our anatomical-based eye model depicts the value of $50 \mathrm{kPa}$. In other parts of the stress-displacement diagrams, the same trend was observed and our model showed a very good agreement compared to both the experimental data and other three models. It should also be pointed out incorporating the mechanical properties of the human sclera under various loading rates into the eye model, helped to have a better numerical simulation with realistic sclera's properties as well as suitable loading rate for a wide range of eye injury simulations. In order to certify the application of our anatomical-based eye model and bring some examples, the injury to the eye as a result of blunt trauma impact as well as IOP variation was investigated. The tennis ball at the speed of $69.29 \mathrm{~m} / \mathrm{s}$ was hit the eye and the stress as a result of that at two different elastic moduli for the sclera/optic nerve tissue. The results in this regard showed a higher stress in the cornea regardless of the variation in the elastic modulus. The amount of stress in the retina, sclera, and optic nerve was affected by the alteration of the elastic 
modulus. However, the most part of the injury as a result of impact was located in the anterior side of the eye. Regarding the IOP, the IOP at three different values, such as 10,20 , and $30 \mathrm{mmHg}$, was applied in the aqueous body and the resultant stresses and deformations in the rest of the components of the eye were calculated. The results in terms of the tennis ball impact showed non-significant role of the sclera/optic nerve elastic modulus on the stress and deformation of the other components of the eye. Regarding the IOP, the results showed the curvature values of 7.89 and $2.50 \mathrm{~mm}$ for the cornea and lens, respectively, as a consequence of IOP value of $30 \mathrm{mmHg}$. The most injured component of the eye among all of them was the cornea with $247.25 \mathrm{kPa}$ at the IOP of $30 \mathrm{mmHg}$. Additionally, the significant role of the sclera's mechanical properties was also well fulfilled in the ONH since the deformation of 10.42 and $16.02 \mu \mathrm{m}$ were observed on the $\mathrm{ONH}$ for the sclera/optic nerve elastic modulus of 5.50 (reference data) and 1.65 $\mathrm{MPa}$ (our experimental data), respectively, and the deformation using our experimental data was closer to the clinical data. The anatomical-based eye model proposed in the concurrent study have implications not only for simulating the eye injury but also for understanding the medical complications in the eye as a result of various types of injuries which are difficult to be diagnosed experimentally. 


\section{References}


[1] Négrel A-D, Thylefors B. The global impact of eye injuries. Ophthalmic Epidemiology 1998;5:143-69.

[2] Kelly S. Serious eye injury in badminton players. $\mathrm{Br} J$ Ophthalmol 1987;71:746-7.

[3] MacEwen CJ. Sport associated eye injury: a casualty department survey. $B r J$ Ophthalmol 1987;71:701-5.

[4] Nelson LB, Wilson TW, Jeffers JB. Eye injuries in childhood: demography, etiology, and prevention. Pediatrics 1989;84:438-41.

[5] Foster CS, de la Maza MS. The sclera: Springer Science \& Business Media; 2013.

[6] Cioffi G, Van Buskirk E. Vasculature of the anterior optic nerve and peripapillary choroid. Glaucomas 1996;1:177-88.

[7] Downs JC. Optic nerve head biomechanics in aging and disease. Exp Eye Res 2015;133:19-29.

[8] Campbell IC, Coudrillier B, Ethier CR. Biomechanics of the posterior eye: a critical role in health and disease. J Biomech Eng 2014;136:021005.

[9] Quant JR, Woo GC. Normal values of eye position and head size in Chinese children from Hong Kong. Optometry Vis Sci 1993;70:668-71.

[10] Uchio E, Ohno S, Kudoh J, Aoki K, Kisielewicz LT. Simulation model of an eyeball based on finite element analysis on a supercomputer. $\mathrm{Br}$ J Ophthalmol 1999;83:1106-11. 
[11] Uchio E, Ohno S, Kudoh K, Kadonosono K, Andoh K, Kisielewicz LT. Simulation of air-bag impact on post-radial keratotomy eye using finite element analysis. J Cataract Refract Surg 2001;27:1847-53.

[12] Stitzel JD, Duma SM, Cormier JM, Herring IP. A nonlinear finite element model of the eye with experimental validation for the prediction of globe rupture. Sae conference proceedings p: sae; 1999; 2002. p. 81-102.

[13] Rossi T, Boccassini B, Esposito L, Iossa M, Ruggiero A, Tamburrelli C, et al. The pathogenesis of retinal damage in blunt eye trauma: finite element modeling. Invest Ophthalmol Vis Sci 2011;52:3994-4002.

[14] Lovald ST, Rau A, Nissman S, Ames N, McNulty J, Ochoa JA, et al. Finite element analysis and experimental evaluation of penetrating injury through the cornea. J Mech Behav Biomed Mater 2017;66:104-10.

[15] Westmoreland BF, Lemp MA, Snell RS. Clinical Anatomy of the Eye. Blackwell Publishers; 1998.

[16] Friberg TR, Lace JW. A comparison of the elastic properties of human choroid and sclera. Exp Eye Res 1988;47:429-36.

[17] Girard M, Suh J-KF, Hart RT, Burgoyne CF, Downs JC. Effects of storage time on the mechanical properties of rabbit peripapillary sclera after enucleation. Cur Eye Res 2007;32:465-70.

[18] Battaglioli J, Kamm R. Measurements of the compressive properties of scleral tissue. Invest Ophthalmol Vis Sci 1984;25:59-65. 
[19] Downs JC, Suh JF, Thomas KA, Bellezza AJ, Hart RT, Burgoyne CF. Viscoelastic material properties of the peripapillary sclera in normal and earlyglaucoma monkey eyes. Invest Ophthalmol Vis Sci 2005;46:540-6.

[20] Wollensak G, Spoerl E. Collagen crosslinking of human and porcine sclera. J Cataract Refract Surg 2004;30:689-95.

[21] Nickerson CS, Park J, Kornfield JA, Karageozian H. Rheological properties of the vitreous and the role of hyaluronic acid. J Biomech 2008;41:1840-6.

[22] Schultz DS, Lotz JC, Lee SM, Trinidad ML, Stewart JM. Structural factors that mediate scleral stiffness. Invest Ophthalmol Vis Sci 2008;49:4232-6.

[23] Jones I, Warner M, Stevens J. Mathematical modelling of the elastic properties of retina: a determination of Young's modulus. Eye 1992;6:556-9.

[24] Bullock JD, Warwar RE, Green WR. Ocular explosions from periocular anesthetic injections: a clinical, histopathologic, experimental, and biophysical study. Ophthalmology 1999;106:2341-53.

[25] Morris HJ, Tang J, Perez BC, Pan X, Hart RT, Weber PA, et al. Correlation between biomechanical responses of posterior sclera and IOP elevations during micro intraocular volume change. Invest Ophthalmol Vis Sci 2013;54:7215-22.

[26] Bellezza AJ, Hart RT, Burgoyne CF. The optic nerve head as a biomechanical structure: initial finite element modeling. Invest Ophthalmol Vis Sci 2000;41:2991-3000.

[27] Sigal IA, Flanagan JG, Ethier CR. Factors influencing optic nerve head biomechanics. Invest Ophthalmol Vis Sci 2005;46:4189. 
[28] Tabernero J, Vazquez D, Seidemann A, Uttenweiler D, Schaeffel F. Effects of myopic spectacle correction and radial refractive gradient spectacles on peripheral refraction. Vis Res 2009;49:2176-86.

[29] Gefen A, Shalom R, Elad D, Mandel Y. Biomechanical analysis of the keratoconic cornea. J Mech Behav Biomed Mater 2009;2:224-36.

[30] Edmund C. Corneal topography and elasticity in normal and keratoconic eyes. A methodological study concerning the pathogenesis of keratoconus. Acta Ophthalmologica Suppl 1988;193:1-36.

[31] Vinciguerra P, Albè E, Trazza S, Rosetta P, Vinciguerra R, Seiler T, et al. Refractive, topographic, tomographic, and aberrometric analysis of keratoconic eyes undergoing corneal cross-linking. Ophthalmology 2009;116:369-78.

[32] Lennerstrand G. Motor units in eye muscles. Basic mechanisms of ocular motility and their clinical implications Pergamon Press, Oxford 1975;19:141. [33] Snell RS, Lemp MA. Clinical anatomy of the eye: Wiley-Blackwell; 1997. [34] Cassin B, Solomon S, Rubin ML. Dictionary of eye terminology: Triad Pub. Co.; 1984.

[35] Woo S-Y, Kobayashi A, Schlegel W, Lawrence C. Nonlinear material properties of intact cornea and sclera. Exp Eye Res 1972;14:29-39.

[36] Eilaghi A, Flanagan JG, Tertinegg I, Simmons CA, Brodland GW, Ethier CR. Biaxial mechanical testing of human sclera. J Biomech 2010;43:1696-701. [37] Ogden RW. Non-linear Elastic Deformations. New York: Dover; 1997. 
[38] Barraglioli J, Kamm R. Measurements of the compressive properties of scleral tissue. Invest Ophthalmol 1 1984:1.

[39] Holzapfel GA. Nonlinear solid mechanics: Wiley Chichester; 2000.

[40] Troyer KL, Puttlitz CM. Human cervical spine ligaments exhibit fully nonlinear viscoelastic behavior. Acta Biomater 2011;7:700-9.

[41] Lucas SR, Bass CR, Salzar RS, Oyen ML, Planchak C, Ziemba A, et al. Viscoelastic properties of the cervical spinal ligaments under fast strain-rate deformations. Acta Biomater 2008;4:117-25.

[42] Scheffe H. A method for judging all contrasts in the analysis of variance. Biometrika 1953;40:87-110.

[43] Pijanka JK, Coudrillier B, Ziegler K, Sorensen T, Meek KM, Nguyen TD, et al. Quantitative mapping of collagen fiber orientation in non-glaucoma and glaucoma posterior human scleraefiber orientation in posterior human sclera. Invest Ophthalmol Vis Sci 2012;53:5258-70.

[44] Asejczyk-Widlicka M, Śródka W, Schachar R, Pierścionek B. Material properties of the cornea and sclera: A modelling approach to test experimental analysis. J Biomech 2011;44:543-6.

[45] Nguyen TD, Coudrillier B, Pijanka JK, Jefferys J, Quigley H, Boote C. Effects of age on the collagen structure and mechanical properties of the human sclera. Invest Ophthalmol Vis Sci 2014;55:4553-. 
[46] Grytz R, Fazio MA, Girard MJ, Libertiaux V, Bruno L, Gardiner S, et al. Material properties of the posterior human sclera. J Mech Behav Biomed Mater 2014;29:602-17.

[47] Karimi A, Navidbakhsh M, Haghi AM, Faghihi S. Measurement of the uniaxial mechanical properties of rat brains infected by Plasmodium berghei ANKA. Proc Inst Mech Eng H. 2013 May;227(5):609-14.

[48] Karimi A, Navidbakhsh M, Yousefi H, Haghi AM, Sadati SA. Experimental and numerical study on the mechanical behavior of rat brain tissue. Perfusion 2014:0267659114522088.

[49] Karimi A, Navidbakhsh M, Faghihi S. A comparative study on plaque vulnerability using constitutive equations. Perfusion 2013:0267659113502835. [50] Vinger. Sports injuries. In: Alber t DM, Jakobiec FA, eds. Principles and practice of ophthalmology. 2 ed: Philadelphia: W.B. Saunders Company. [51] He M, Wang D, Console JW, Zhang J, Zheng Y, Huang W. Distribution and heritability of iris thickness and pupil size in Chinese: the Guangzhou Twin Eye Study. Invest Ophthalmol Vis Sci 2009;50:1593-7.

[52] Kolb H, Fernandez E, Nelson R. Facts and Figures Concerning the Human Retina--Webvision: The Organization of the Retina and Visual System. 1995.

[53] Vurgese S, Panda-Jonas S, Jonas JB. Scleral thickness in human eyes. PloS One 2012;7:e29692. 
[54] Karimi A, Navidbakhsh M, Razaghi R, Haghpanahi M. A computational fluid-structure interaction model for plaque vulnerability assessment in atherosclerotic human coronary arteries. J Appl Phys 2014;115:144702-11.

[55] Donea J, Huerta A, Ponthot J-P, Rodriguez-Ferran A. Encyclopedia of Computational Mechanics Vol. 1: Fundamentals., Chapter 14: Arbitrary Lagrangian-Eulerian Methods. Wiley \& Sons; 2004.

[56] Karimi A, Navidbakhsh M, Razaghi R. An experimental-finite element analysis on the kinetic energy absorption capacity of polyvinyl alcohol sponge. Mater Sci Eng C 2014;39:253-8.

[57] Schutte S, van den Bedem SPW, van Keulen F, van der Helm FCT, Simonsz HJ. A finite-element analysis model of orbital biomechanics. Vis Res 2006;46:1724-31.

[58] Rangarajan N, Kamalakkannan SB, Hasija V, Shams T, Jenny C, Serbanescu I, et al. Finite element model of ocular injury in abusive head trauma. $J$ AAPOS 2009;13:364-9.

[59] Karimi A, Razaghi R, Navidbakhsh M, Sera T, Kudo S. Mechanical properties of the human sclera under various strain rates: Elastic, hyperelastic, and viscoelastic models. J Biomater Tissue Eng. In Press.

[60] Anderson K, El-Sheikh A, Newson T. Application of structural analysis to the mechanical behaviour of the cornea. J Royal Society Interface 2004;1:3-15. 
[61] Asejczyk-Widlicka M, Śródka D, Kasprzak H, Pierscionek B. Modelling the elastic properties of the anterior eye and their contribution to maintenance of image quality: the role of the limbus. Eye 2007;21:1087-94.

[62] Hallquist JO. LS-DYNA theory manual. Livermore software Technology corporation 2006;3:25-31.

[63] Karimi A, Navidbakhsh M, Rahmati SM, Sera T, Kudo S. A combination of experimental and numerical methods to investigate the role of strain rate on the mechanical properties and collagen fiber orientations of the healthy and atherosclerotic human coronary arteries. Bioengineered 2016.

[64] Ayyalasomayajula A, Park RI, Simon BR, Vande Geest JP. A porohyperelastic finite element model of the eye: the influence of stiffness and permeability on intraocular pressure and optic nerve head biomechanics. Comput Methods Biomech Biomed Engin 2016;19:591-602.

[65] Weaver AA, Stitzel SM, Stitzel JD. Injury risk prediction from computational simulations of ocular blast loading. Biomech Model Mechanobiol 2016:1-15.

[66] Voorhies KD. Static and dynamic stress/strain properties for human and porcine eyes: Virginia Polytechnic Institute and State University; 2003.

[67] Beswick J, McCulloch C. Effect of hyaluronidase on the viscosity of the aqueous humour. Br J Ophthalmol 1956;40:545.

[68] Whitcomb JE, Barnett VA, Olsen TW, Barocas VH. Ex vivo porcine iris stiffening due to drug stimulation. Exp Eye Res 2009;89:456-61. 
[69] Power ED. A nonlinear finite element model of the human eye to investigate ocular injuries from night vision goggles. 2001.

[70] Czygan G, Hartung C. Mechanical testing of isolated senile human eye lens nuclei. Med Eng Phys 1996;18:345-9.

[71] Wollensak G, Spoerl E. Biomechanical characteristics of retina. Retina 2004;24:967-70.

[72] Cao YP, Lu J. A new method to extract the plastic properties of metal materials from an instrumented spherical indentation loading curve. Acta Materialia 2004;52:4023-32.

[73] Lee B, Litt M, Buchsbaum G. Rheology of the vitreous body. Part I: Viscoelasticity of human vitreous. Biorheology 1991;29:521-33.

[74] Schoemaker I, Hoefnagel PP, Mastenbroek TJ, Kolff CF, Schutte S, van der Helm FC, et al. Elasticity, viscosity, and deformation of orbital fat. Invest Ophthalmol Vis Sci 2006;47:4819-26.

[75] Karimi A, Rahmati SM, Navidbakhsh M. Mechanical characterization of the rat and mice skin tissues using histostructural and uniaxial data. Bioengineered 2015;6:153-60.

[76] Zhang K, Qian X, Mei X, Liu Z. An inverse method to determine the mechanical properties of the iris in vivo. Biomed Eng Online 2014;13:66.

[77] Négrel A-D, Thylefors B. The global impact of eye injuries. Ophthalmic Epidemiology 1998;5:143-69. 
[78] Buhr ED, Yue WW, Ren X, Jiang Z, Liao H-WR, Mei X, et al. Neuropsin (OPN5)-mediated photoentrainment of local circadian oscillators in mammalian retina and cornea. Proc Nat Acad Sci 2015:201516259.

[79] Bradbury M, Cole D. The role of the lymphatic system in drainage of cerebrospinal fluid and aqueous humour. J Physiol 1980;299:353-65.

[76] Shin TJ, Vito RP, Johnson LW, McCarey BE. The distribution of strain in the human cornea. J Biomech 1997;30:497-503.

[77] Hukins DW, Aspden RM. Composition and properties of connective tissues. Trends in Biochem Sci 1985;10:260-4.

[78] Tuori AJ, Virtanen I, Aine E, Kalluri R, Miner J, Uusitalo HM. The immunohistochemical composition of corneal basement membrane in keratoconus. Cur Eye Res 1997;16:792-801.

[79] Cheng EL, Maruyama I, SundarRaj N, Sugar J, Feder RS, Yue BY. Expression of type XII collagen and hemidesmosome-associated proteins in keratoconus corneas. Cur Eye Res 2001;22:333-40.

[80] Pieh S, Marvan P, Lackner B, Hanselmayer G, Schmidinger G, Leitgeb R, et al. Quantitative performance of bifocal and multifocal intraocular lenses in a model eye: point spread function in multifocal intraocular lenses. Arch Ophthalmol 2002;120:23-8.

[81] Ware C. Information visualization: perception for design: Elsevier; 2012. 
[82] Fisher R. The ciliary body in accommodation. Trans Ophthalmol Soc UK 1985;105:208-19.

[83] Findl O, Kiss B, Petternel V, Menapace R, Georgopoulos M, Rainer G, et al. Intraocular lens movement caused by ciliary muscle contraction. J Cataract Refract Surg 2003;29:669-76.

[84] Barrell G, Cooper P, Elkington A, Macfadyen J, Powell R, Tormey P. Squash ball to eye ball: the likelihood of squash players incurring an eye injury. $B M J$ $1981 ; 283: 893-5$.

[85] Jones MNP. Eye injury in sport. Sports Medicine 1989;7:163-81.

[86] Rubin ML. Perspectives in refraction. Surg 1988;32:357-60.

[87] Gregory P. Sussex Eye Hospital sports injuries. $\mathrm{Br} J$ Ophthalmol 1986;70:748-50.

[88] Shin TJ, Vito RP, Johnson LW, McCarey BE. The distribution of strain in the human cornea. J Biomech 1997;30:497-503.

[89] Hukins DW, Aspden RM. Composition and properties of connective tissues. Trends Biochem Sci 1985;10:260-4.

[90] Tuori AJ, Virtanen I, Aine E, Kalluri R, Miner J, Uusitalo HM. The immunohistochemical composition of corneal basement membrane in keratoconus. Cur Eye Res 1997;16:792-801.

[91] Cheng EL, Maruyama I, SundarRaj N, Sugar J, Feder RS, Yue BY. Expression of type XII collagen and hemidesmosome-associated proteins in keratoconus corneas. Cur Eye Res 2001;22:333-40. 
[92] Emara B, Probst LE, Tingey DP, Kennedy DW, Willms LJ, Machat J. Correlation of intraocular pressure and central corneal thickness in normal myopic eyes and after laser in situ keratomileusis. J Cataract Refract Surg 1998;24:1320-5.

[93] Meek KM, Boote C. The organization of collagen in the corneal stroma. Exp Eye Res 2004;78:503-12.

[94] Pierscionek BK, Asejczyk-Widlicka M, Schachar RA. The effect of changing intraocular pressure on the corneal and scleral curvatures in the fresh porcine eye. Br J Ophthalmol 2007;91:801-3.

[95] Liu J, Roberts CJ. Influence of corneal biomechanical properties on intraocular pressure measurement: quantitative analysis. J Cataract Refract Surg 2005;31:146-55.

[96] Eysteinsson T, Jonasson F, Sasaki H, Arnarsson A, Sverrisson T, Sasaki K, et al. Central corneal thickness, radius of the corneal curvature and intraocular pressure in normal subjects using non-contact techniques: Reykjavik Eye Study. Acta Ophthalmologica Scandinavica 2002;80:11-5.

[97] Krachmer JH, Feder RS, Belin MW. Keratoconus and related noninflammatory corneal thinning disorders. Survey Ophthalmology 1984;28:293-322.

[98] Sakamoto Y, Sasaki K. Accuracy of Biometrical Data Obtained from the NIDEKEAS-1000. Ophthalmic Res 1994;26:26-32. 
[99] Burns DM, Johnston FM, Frazer DG, Patterson C, Jackson AJ. Keratoconus: an analysis of corneal asymmetry. Br J Ophthalmol 2004;88:1252-5.

[100] Duncan JK, Belin MW, Borgstrom M. Assessing progression of keratoconus: novel tomographic determinants. Eye Vis 2016;3:6.

[101] Zuckerman A. Occular injuries resulted from the war. Trans Opthal Soc UK 1941;61:45-50.

[102] Bryant MR, McDonnell PJ. Constitutive laws for biomechanical modeling of refractive surgery. J Biomech Eng 1996;118:473-81.

[103] Burgoyne CF, Crawford Downs J, Bellezza AJ, Francis Suh JK, Hart RT. The optic nerve head as a biomechanical structure: a new paradigm for understanding the role of IOP-related stress and strain in the pathophysiology of glaucomatous optic nerve head damage. Progress Retinal Eye Res 2005;24:3973.

[104] Levy NS, Crapps EE. Displacement of optic nerve head in response to short-term intraocular pressure elevation in human eyes. Arch Ophthalmol 1984;102:782-6.

[105] Levy NS, Crapps EE, Bonney RC. Displacement of the optic nerve head: response to acute intraocular pressure elevation in primate eyes. Arch Ophthalmol 1981;99:2166-74. 


\section{ACKNOWLEDGMENTS}

Firstly, I would like to express my sincere gratitude to my advisors Professor Susumu KUDO and Associate Professor Toshihiro SERA for the continuous support of my Ph.D study and related research, for their patience, motivation, and immense knowledge. Their guidance helped me in all the time of research and writing of this thesis. I could not have imagined having better advisors and mentors for my Ph.D study.

Besides my advisors, I would like to thank the committee of my thesis: Professor Yoshinori SAWAE, and Associate Professor Mitsugu TODO, for their insightful comments and encouragement, but also for the hard question which incented me to widen my research from various perspectives. We also thank to our collaborators in Iran, especially Dr. Navidbakhsh and Reza Razaghi, for their help.

I thank my labmates in Biofunctional Engineering Laboratory for their help in Japanese language and for all the fun we have had in the last two years. Furthermore, I thank to my friends in Kyushu University.

I am also grateful to financial support by Japanese Government (Monbukagakusho) Scholarship.

Last but not the least, I would like to thank my family: my parents and my sisters, for supporting me spiritually throughout writing this thesis and my life in general.

Alireza Karimi

Kyushu University

March 2017 